\title{
Spatial Distortions in Visual Short-Term Memory
}

\author{
Dissertation \\ zur Erlangung des Doktorgrades \\ der Mathematisch-Naturwissenschaftlichen Fakultäten \\ der Georg-August-Universität zu Göttingen
}

vorgelegt von

Thomas Schmidt

aus Braunschweig

Göttingen 2001 


\section{Acknowledgements}

Many people have helped me working out the results reported in this thesis. First of all, I want to thank the students who form the heart and soul of our research project: in alphabetical order, Sabine Bakkes (sharing my Suzanne Vega addiction), Jennifer Fehr (for funny confusions of spatial reference systems), Antje Fillbrandt (collecting new psychometric functions), Andreas Finkelmeyer (great tips in electronic music), Melanie Jäger (relentlessly trying to confuse poor map-reading students), Vanessa Jainek (for funny jumps during a nightly horror movie session), Eun Young Lee (for giving me a chance to show off with my home improvement skills), Ralf Mayrhofer (the master of machine language), Björn Rump (boldly listening to music that noone has called "music" before), Kristine Schmidt (no, we are not married), Kami Schott (for sort of improving my English and stuff), and Claudia Wilimzig (always my favorite victim for sharing new data) who also proofread the final version of this thesis. You are the living proof that science must be fun! Thanks also to the students who assisted in my teaching: Björn Albrecht, Vivien Kurtz, Annika Mundhenke (the only person who seems to like to watch my "Buffy" videos), Johann Weichbrodt, and all the students who made our lab courses and methodology lectures so rewarding.

A special thank you goes to Julia Trommershäuser for solving all our theoretical problems. Sometimes it takes a quantum physicist! I also thank my colleague Nuria Vath for her great dedication in running our EEG lab, and Peter Breuer for always being there to rescue our equipment.

This thesis builds on the work of Jörn Diedrichsen, whom I am much indebted; not only for his seemingly endless record of pioneering data on spatial memory distortions, but also for many important discussions which always generated even more light than heat...!

I want to thank all the friends and colleagues who discussed our data with us, especially Dirk Vorberg and Jochen Müsseler. Thanks also to Armin Heinecke, Heiner Deubel, Satoru Suzuki, Bill Prinzmetal, Dale Dagenbach and all the people who came to our talks and posters. Thanks to Michael Waldmann for helpful comments which (so I hope) did much to improve the final version of this paper.

Finally, a very special Thank You goes to Prof. Dr. Gerd Lüer for the great help and support he provided for our research and for myself personally. Without his trust in our scientific enterprise, this project would never have been possible.

A preliminary draft of this thesis was completed in the house of Steffen, Nicole, and Lena Werner, in Moscow, Idaho - thanks for the hospitality and a beautiful week. Even more than all the persons mentioned so far, I thank Steffen Werner for his support and friendship; and for giving me the chance to work in his laboratory at a critical point in my career without asking for my reputation.

Göttingen, October 2001. 


\section{Publishing note:}

Experiments 3 and 4 were published in Werner and Schmidt (2000). Experiments 5 and 6 will (so we hope) be reported in a forthcoming paper (Schmidt, Diedrichsen \& Werner, submitted). Here, additional data and new statistical analyses are provided. I heartily thank my coauthors Steffen and Jörn for their help and intellectual input to these and other parts of this manuscript.

This work was supported by a grant of the German Research Community (We 1973/3) to Steffen Werner. 
Meiner Familie 
"Science must be fun!"

Patricia Churchland, personal communication, mistaking the author

for a philosopher of the same name.

\section{Contents}

Acknowledgements I

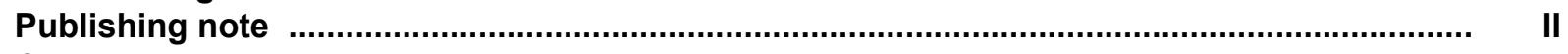

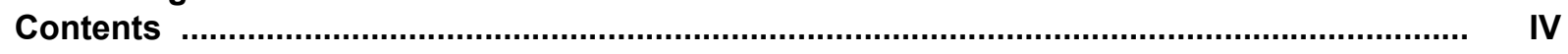

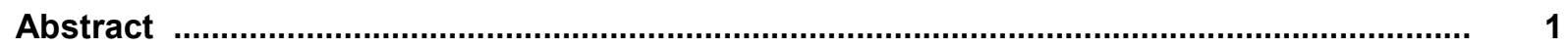

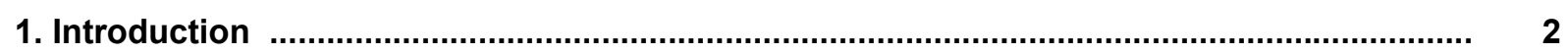

1.1 Spatial Representations ............................................................................... 2

1.1.1 A cartographer's brain ....................................................................... 2

1.1.2 Coding of spatial object features ......................................................... 3

1.1.3 Neural correlates of spatial short-term memory ...................................... 5

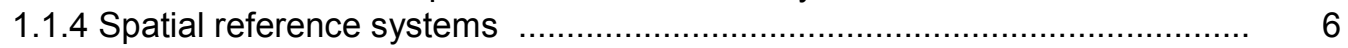

1.2 Distortions in Memory for Two-Dimensional Layout .......................................... $\quad 7$

1.2.1 Spatial representation of two-dimensional layout ...................................... 7

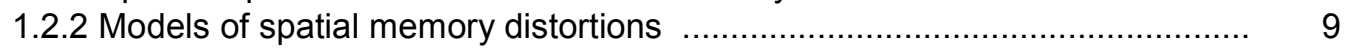

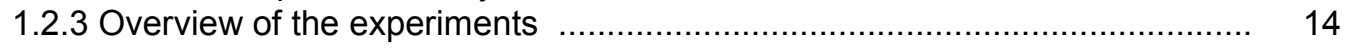

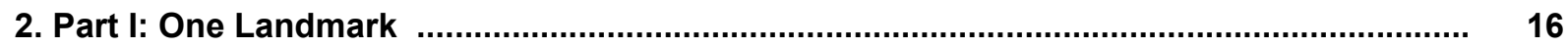

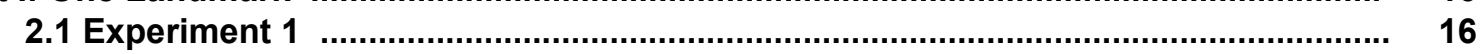

2.1.1 Method .................................................................... 17

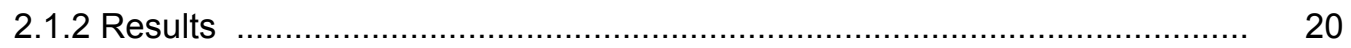

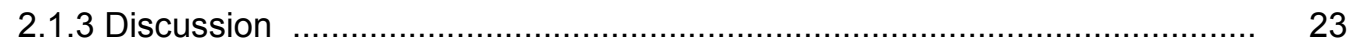

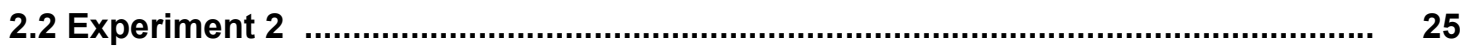

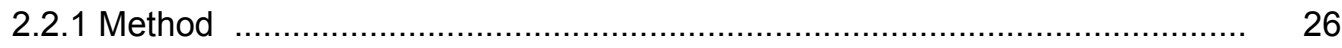

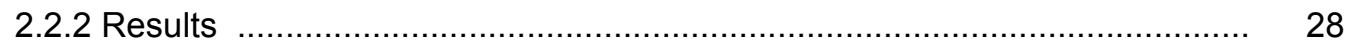

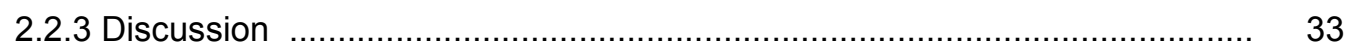

(continued on following page) 
(continued)

3. Part Ila: Two Landmarks - Orientational Invariance and the Time-Course of Distortion..$\quad 35$

3.1 Experiment 3 ................................................................................................. 35

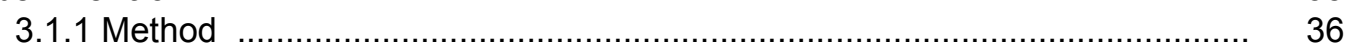

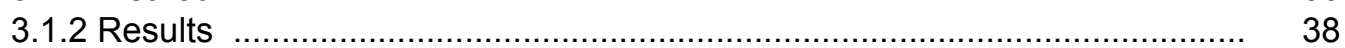

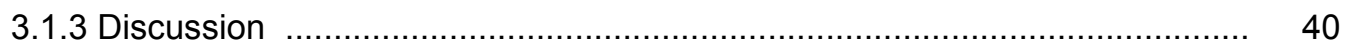

3.2 Experiment 4 ......................................................................................... 41

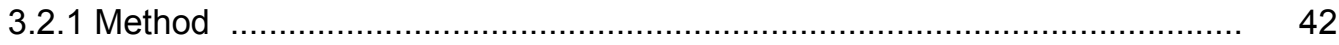

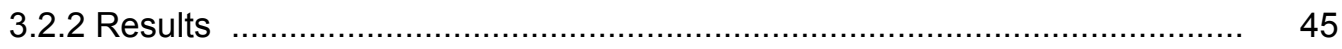

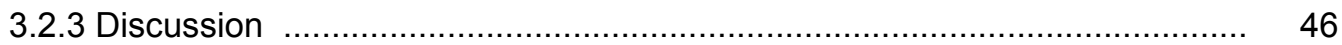

4. Part Ilb: Two Landmarks - Nonlinear Organisation of the Visual Field ............................ 49

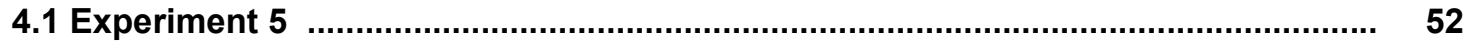

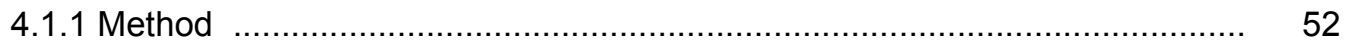

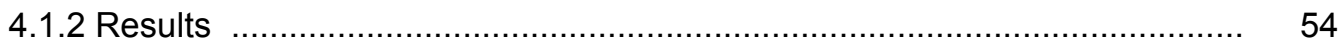

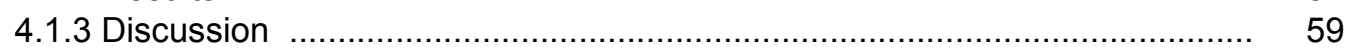

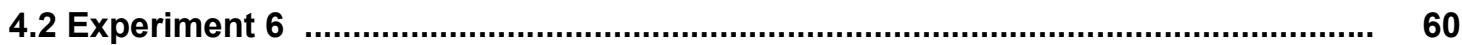

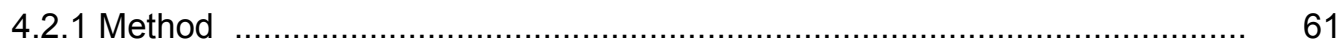

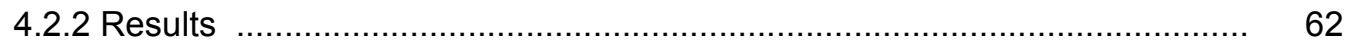

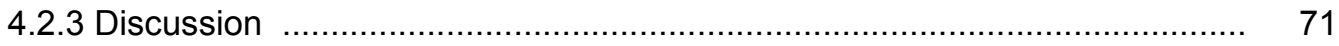

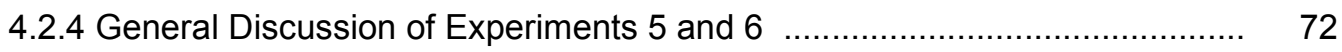

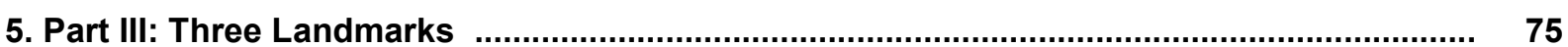

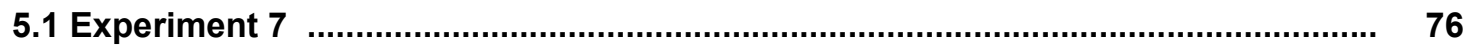

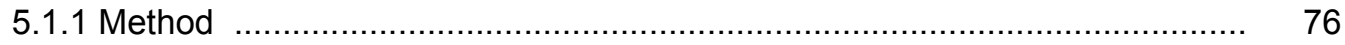

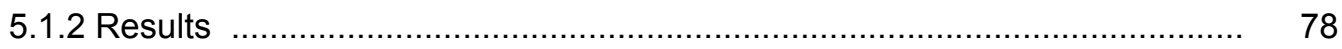

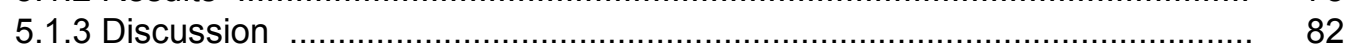

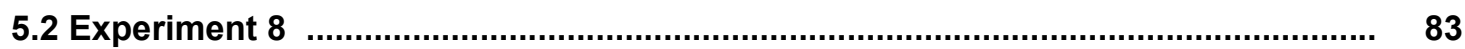

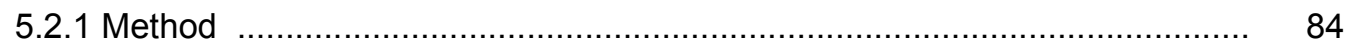

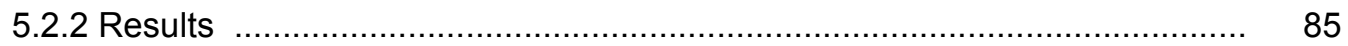

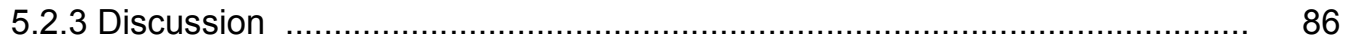

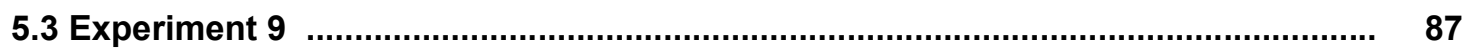

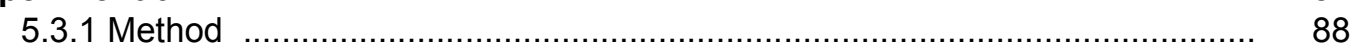

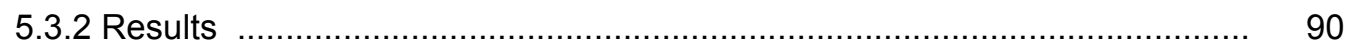

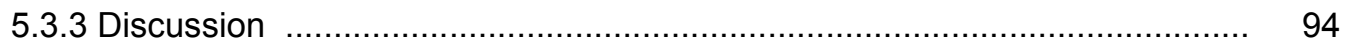

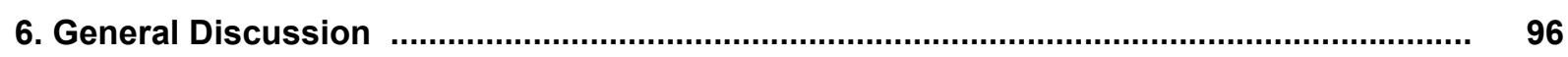

6.1 Reduction of spatial uncertainty and the origin of spatial distortions ............... 96

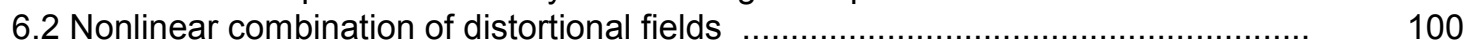

6.3 Interactions of intrinsic and extrinsic reference systems ........................... 103

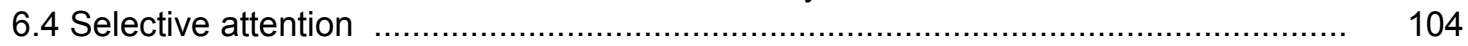

6.5 An attentional preactivation model of spatial memory distortions .................... 106

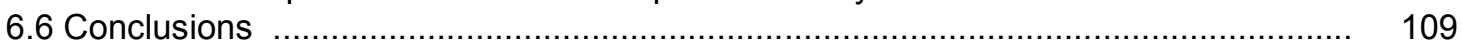

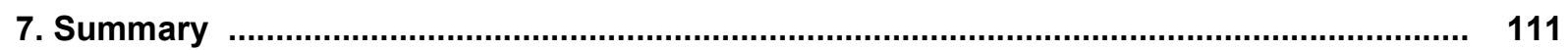

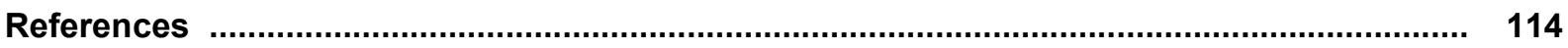

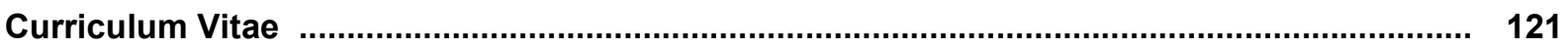




\begin{abstract}
Two-dimensional topographic maps represent a major coding principle for spatial information in the primate brain. In principle, neuronal maps can be subject to systematic distortion caused by interactions between neighboring cells as well as attentional influences. In this thesis, it is shown that when observers try to reproduce the exact position of a target dot on the monitor screen after a brief retention interval, their memory is systematically distorted by the presence of visual landmark stimuli serving as spatial reference. Landmarks provide regions of high positional certainty, markedly reducing response variance in their vicinity. At the same time, configurations of one, two, or three landmarks induce predictable distortional fields that are governed mainly by a stimulus-based (intrinsic) frame of reference but also interact with extrinsic reference systems, e.g., the allocentric vertical. Distortional fields can be invariant with image transformations, closely following changes in orientation, translation, or elongation of the landmark configuration, and are established as soon as $100 \mathrm{~ms}$ after the target stimulus has disappeared. Furthermore, it is shown that the distortional field of two landmarks can be predicted on the basis of knowledge of the single landmarks presented individually because the fields are locally invariant when a second landmark is presented at some distance, which leads to a partitioning of the visual field into regions of influence dominated by single landmarks. These results are inconsistent with previous theories of spatial memory distortions. Instead, an attentional model is advanced where spatial distortions arise from a preactivation of spatial reference systems in topographical cortical memory maps.
\end{abstract}




\section{Introduction}

\subsection{Spatial Representations}

\subsubsection{A cartographer's brain}

Vision scientists widely agree that "space is special", i.e., that the spatial location of an object is not just one feature among others, like its color, form, or orientation. Instead, the spatial organisation of features is what defines an object in the first place (Palmer, 1999). With the possible exception of color, all visual object features have some kind of spatial organization, and an object is uniquely defined by the spatial arrangement of its primitives. This is also true of complex objects with discernible parts: whether an object is immediately recognizable as a functionable piece of household equipment or just forms an unrecognizable cluster of steel sheets and plastic depends critically on the spatial arrangement of its parts. Modern theories of object perception stress the point that complex objects should be regarded as assemblies of simple geometrical primitives in suitable spatial arrangement (Marr \& Nishihara, 1978; Biederman, 1987).

Many of the problems that make visual perception tricky are spatial in nature. For example, the brain faces the complicated task of inferring the three-dimensional spatial layout of its environment from the two-dimensional image on the retina. To do so, it uses several sources of indirect information (like shading or texture gradients), along with heuristic principles that allow for reconstruction of the third dimension (Hershenson, 1999). However, the raw material for these reconstructional processes, the perception of objects as well as that of three-dimensional layout, remains organized in two-dimensional representations, so-called maps, throughout most of visual processing. Thus, the fundamental properties of those maps can influence all subsequent stages of human vision.

Spatial maps are abundant in the primate brain. Starting from early representations in the retina, maps tend to be topographic and, in terms of neuronal connections, in alignment with each other. As early vision proceeds from the retina via the lateral geniculate nucleus of the thalamus (LGN) to primary visual cortex (V1), more and more processing ressources are devoted to the central, macular part of the visual field. After the retina, V1 is probably the most important relay station for visual signals because it is holds a "master map" of the entire visual field where informa- 
tions from the two eyes are first combined, and because it serves as a distributor for visual features like lines and edges, spectral information, and motion, that are passed on to later, more specialized areas (Livingstone \& Hubel, 1987, 1988).

As one ascends the hierarchy of visual areas, receptive fields in the retina, the LGN, and V1 are in spatial register and increase in size, redundantly covering the entire visual field. Many authors regard these cells as performing some kind of spatial filtering operation and assume that they are retrieving information from different scales of spatial frequency (Blakemore \& Campbell, 1969; DeValois \& DeValois, 1988), a view voiced most eloquently by Marr (1982). Spatial filtering is an efficient coding strategy for the visual system because resulting maps of different spatial frequencies can later be combined again with very little loss of information, thus simplifying the task of representing a complex visual image. Topographical coding is maintained in early areas further downstream, e.g., V2, V3, and V3A.

\subsubsection{Coding of spatial object features}

Beyond the early visual areas, areas begin to specialize in function. Recently, the view has become popular that visual processing proceeds in two distinct parts of the visual system (Ungerleider \& Mishkin, 1982; Felleman \& Van Essen, 1991; for a critical review see Merigan \& Maunsell, 1993). In this view, there is a ventral stream of visual processing running from early areas via $\mathrm{V} 4$ to inferotemporal cortex, and a dorsal stream running via area MT to posterior parietal cortex. These streams are supposed to subserve different functions, the ventral stream (the "what" system) being concerned with object recognition and the dorsal stream (the "where" system) being responsible for analyzing and transforming spatial relationships. Topographical maps are present in most areas of the dorsal stream and some earlier areas of the ventral stream, although ventral-stream cells further downstream tend to respond to object features or even identity rather than object location.

Early evidence for this view came from lesion studies that found deficits in a spatial task in monkeys with lesions in dorsal areas, and deficits in object recognition following ventral lesions (Ungerleider \& Mishkin, 1982). More recently, Milner and Goodale (1995; Goodale \& Milner, 1992) have advanced a reinterpretation of classical two-visual-systems theory. They maintain the view of a ventral processing stream which is concerned with object recognition, memory encoding, and visual awareness, but reinterpret the function of the dorsal stream. In their view, the dorsal pathway is a 
"how" system rather than a "where" system, dealing with fast, immediate visuomotor transformations instead of spatial processing per se. Typical examples of tasks for dorsal-stream circuitry include the on-line visual control of grasping movements, pointing movements, and saccades; e.g., when catching a ball in flight or when reaching out to grasp a coffee cup. Because of their specialized visuomotor circuitry, transformations in the dorsal stream can occur very quickly and are not dependent on the time-consuming process of building consciously accessible visual representations in the ventral stream (Milner \& Goodale, 1995).

The most important evidence for two-visual-systems theory comes from patients with lesions in either dorsal or ventral stream areas. For example, the intensely studied patient D.S. (Goodale, Milner, Jakobson, \& Carey, 1991; Milner et al., 1991) suffers from severe visual form agnosia because of ventral pathway lesions following carbon monoxide intoxication. She is not able to recognize or describe visual objects. However, she is surprisingly good at using these objects for visuomotor tasks, e.g., shaping her fingers to grasp them appropriately. This suggests that her visuomotor system is still largely intact, enabling her to use intrinsic spatial features of the object like its cardinal axis or its center of mass. In contrast, patients with lesions in dorsal stream areas are not impaired in object recognition but are severely handicapped when asked to use objects for motor actions, a complex syndrome called optic ataxia (Perenin \& Vighetto, 1988). Together, visual form agnosia and optic ataxia form a double dissociation of dorsal and ventral stream function, suggesting that the two systems work independently.

There are some indications that the dorsal and ventral systems can also become dissociated in neurologically unimpaired observers. For example, there is evidence that some visual illusions affect visuomotor tasks less than traditional psychophysical tasks. Aglioti, DeSouza, and Goodale (1995) showed that the diameter of the central circle in the Titchener illusion was systematically misperceived depending on the diameters of the outer circles. However, when asked to grasp the central circle, participants' grip size aperture was largely unaffected by the illusion (see also Haffenden \& Goodale, 1998). Similar dissociations were found in tasks where the apparent position of a target in a rectangular frame was biased by a sudden translation of the frame, creating substantial perceptual mislocalization of the target but much smaller biases in visuomotor responses (Bridgeman, Gemmer, Forsman, \& Huemer, 2000; Bridgeman, Peery, \& Anand, 1997). However, dissociations between 
perceptual and visuomotor measures of visual illusions could not always be replicated, and there are concerns about the validity of these results (Franz, Gegenfurtner, Bülthoff \& Fahle, 2000).

\subsubsection{Neural correlates of spatial short-term memory}

Whatever the exact spatial coding scheme in posterior cortical areas might be, perceived stimulus locations must eventually be transformed into motor coordinates, e.g., when keeping a stimulus position in short-term memory to guide a subsequent pointing response. Psychological studies of short-term memory have hinted at a specialized visual buffer where spatial information can be stored and manipulated (Phillips \& Baddeley, 1971; Baddeley, 1990). Single-cell recordings from alert monkeys strongly suggest that dorsolateral prefrontal cortex (dIPFC) is able to store a distributed representation of target location for several seconds (Goldman-Rakic, Ó Scalaidhe \& Chafee, 2000). In these experiments, a monkey is presented with an array of spatial targets that disappears after an inspection interval. The monkey's task is to hold the target in working memory and then perform an eye movement towards it after a start signal. A subset of cells in dIPFC is not only active while the stimulus is presented, but also during the delay period before the start signal occurs. (Funahashi, Bruce, \& Goldman-Rakic, 1989). In turn, if the acitivity of these cells is not maintained during the retention interval, the monkey is highly likely to produce an error.

Like earlier topographical representations, dIPFC has a columnar organization where clusters of adjacent cells respond selectively to certain locations in the visual field. Temporary inactivation of such clusters leads to selective loss of spatial working memory for corresponding regions of the visual field (Sawaguchi \& Goldman-Rakic, 1991). Dorsolateral prefrontal cortex has strong and direct connections to posterior parietal cortex (Goldman-Rakic, 1995; Fuster, 1988), and cells within area 7 of intraparietal cortex and area 8a of dIPFC tend to fire in tandem (Chafee \& GoldmanRakic, 1998). Ultimately, of course, the crucial brain area for executing responses other than eye movements is primary motor cortex. Here, cells also have a distributed code of the spatial location of the target such that spatial tuning curves of individual cells are vectorially combined in a population response (Georgopoulos, Schwartz, \& Kettner, 1986; Georgopoulos, Lurito, Petrides, Schwartz, \& Massey, 1989). Dorsolateral prefrontal areas are intimately linked to motor and premotor ar- 
eas (Passingham, 1995), so that there is an uninterrupted pathway going from early visual areas via posterior parietal cortex to dIPFC and ultimately to executive motor areas, forming a network specialized in the on-line handling of spatial information. Topographical maps seem to present the ubiquitous coding principle within this pathway.

\subsubsection{Spatial reference systems}

In spite of the early distinction between "where" and "what" (or "what" and "how") systems, it is important to recognize that both object recognition and visuomotor control require extensive spatial processing in a variety of spatial reference systems. For object recognition, it is crucial to represent the various parts of the object in correct spatial relationships; it is also important to localize objects with respect to each other. Marr (1982) even suggested that the ultimate goal of vision is a spatial description of objects in an object-based frame of reference that is independent of the observer's location and orientation.

In contrast, visuomotor control has very different requirements with respect to spatial reference systems. For example, to return a tennis ball one has to represent its trajectory with respect to one's own body. This is achieved by a series of transformations, starting with a retinocentric spatial code and advancing to head-centered, hand-centered, and possibly shoulder-centered reference frames found, e.g., in posterior parietal area 7a (Colby, Duhamel, \& Goldberg, 1995; Soechting \& Flanders, 1992; Flanders, Helms Tillery, \& Soechting, 1992). Milner and Goodale's (1995) twovisual-systems theory carefully distinguishes between allocentric reference systems based on relative spatial positions of objects with respect to each other and egocentric reference systems based on the position of objects relative to the body, strictly assigning allocentric coding to the ventral and egocentric coding to the dorsal system. However, this does not mean that spatial object properties are not processed in the dorsal stream because many visuomotor tasks imply the correct identification of centers of mass, points of leverage and other object features (consider, for example, the delicate exercise of juggling with burning torches). Just as egocentric reference systems can be subdivided into retina-centered, head-centered, or hand-centered as well as many other possible coordinate frames, allocentric reference systems must be subdivided into different classes depending on their anchoring point, their orientation, and the geometrical structure of their axes. 
Neurological evidence for possible dissociations of spatial reference frames comes from a common syndrome called hemineglect, often following from lesions of posterior parietal areas (Halligan \& Marshall, 1994). Patients with this type of lesion (often acquired after a stroke) have difficulty attending to the contralesional visual half-field. Importantly, neglect can occur in a variety of spatial reference systems. While some patients simply ignore all parts of visual space that lie to the left of their line of sight (thus using a retinotopic reference system), other patients ignore the left half of their body, e.g., when shaving or applying make-up (using a body-centered reference system), and still other patients ignore the left half of each visual object (using intrinsic, object-based allocentric reference systems, e.g., the axis of elongation). It is still debated whether neglect is a disorder of space representation or visual attention (Halligan \& Marshall, 1994), but its occurence in different spatial reference systems certainly suggests the simultaneous use of such reference systems in the brain.

In this thesis, I will often distinguish between intrinsic and extrinsic reference systems. Intrinsic systems are defined by cardinal axes of a visual object, whereas extrinsic systems are defined by any reference frame independent of the object. Such a reference frame might be allocentric (e.g., the gravity axis), or egocentric (e.g., the observer's body axis).

\subsection{Distortions in Memory for Two-Dimensional Layout}

\subsubsection{Spatial representation of two-dimensional layout}

When we are looking at a visual scene, we have no difficulty figuring out the exact spatial relations between objects. Despite a large compression of visual space along the depth axis (Wagner, 1985; Norman, Todd, Perotti, \& Tittle, 1996), at least in the frontoparallel plane (say, a photograph) the relative distances between everyday objects seem to be perceived accurately. For example, the estimation of line length is so precise that this task is presented as a control task in psychophysical experiments because observers are almost perfect here, psychophysical functions having a Stevens' exponent near 1 (Gescheider, 1997; Baird, 1970). Thus, line length is among the very rare stimulus properties that are perceived veridically.

However, stimuli presented in psychophysical laboratories tend to be wellcontrolled but very impoverished. There is abundant evidence that even the veridical- 
ity of line length estimation can break down dramatically as soon as stimuli are presented within a visual context. As everyone knows from introductory psychology textbooks, perceived line length in a Müller-Lyer stimulus is severely distorted by the presence of suitable flanking lines (e.g., Crawford, Huttenlocher \& Engebretson, 2000). Other visual illusions lead to biased perception of angles, size, parallelism, and other fundamental aspects of spatial layout. These visual illusions have led some authors to assume that distortions in space perception are actually the rule rather than the exception. For example, Watson (1977) has suggested that all visual stimuli induce a curvature of visual space in the same fashion that masses induce a curvature of physical space in Einsteinian physics. According to this view, every line or dot that enters the visual field is a source of distortion; it might cause nearby lines to curve towards it or away from it, or shift the positions of line terminations. Although Watson's (1977) account is purely descriptive and offers no explanation as to what causes perceptual space to bend in the first place, it is able to account for a range of visual illusions and predicts correctly that some illusions will increase in strength as the energy of the inducing lines increases.

There is a number of psychophysical studies investigating properties of spatial coding in visual short-term memory. Spatial distortions are observed when the position of a small target in the two-dimensional plane is remembered (rather than perceived) in the presence of other stimuli. For example, Nelson and Chaiklin (1980) presented a circle with a target dot located somewhere on a visible diameter line. Participants viewed this display for one second and then tried to reproduce the exact location of the target dot on the diameter line. Responses were systematically distorted away from the center of the circle, with an additional distortion towards the center for points near the circumference line, provided that participants had enough time to inspect the display. The distortional pattern was sensitive to changes in the geometry of the stimulus: when the circle was changed into an ellipse, errors were more pronounced along the longer axis of the ellipse.

Huttenlocher, Hedges, and Duncan (1991; see also Laeng, Peters, \& McCabe, 1998) also presented a single target dot within a large circle, but in contrast to Nelson and Chaiklin's procedure, participants' responses were not restricted to a diameter line but were free to depart from the target in any direction. The clustering of responses suggested that participants encoded stimulus positions in a representation similar to a polar coordinate system centered on the circle, with independent biases 
in the angular and radial coordinates. Additionally, participants seemed to spontaneously segment the circle into quadrants by the subjective horizontal and vertical meridian lines since responses were systematically biased away from these lines.

In the displays used by Nelson and Chaiklin (1980) and Huttenlocher et al. (1991), landmark stimuli consisted of a set of points in visual space arranged in a geometric figure (a circle or ellipse). In previous experiments in our laboratory, we have used an even simpler configuration of only two landmark dots, one to the left and one to the right of the display center, in an otherwise empty display (Diedrichsen, 1998; Diedrichsen \& Werner, submitted; Werner \& Schmidt, 2000). Participants had to remember the exact location of a briefly presented target dot and then reproduce it after a short retention interval, using a mouse cursor or stylus. We found that two visual landmarks presented during the trial induced a systematic pattern of spatial biases that we call a distortional field: targets near the landmarks were reproduced too far away from them, and often there was an additional bias away from the midpoint between the landmarks.

In an extensive series of experiments, Diedrichsen (1998) explored the properties of distortional fields in short-term visual memory. He found that increasing or decreasing the distance between the landmarks led to expansion or compression of the distortional field, suggesting that distortions occurred within an intrinsic, stimuluscentered system of reference and that biomechanical artefacts could not account for the effect. When the landmarks were shifted away from their original position and participants were asked to reproduce the target location with respect to the landmarks, distortional fields were also unaffected. Distortions became larger with increasing masking interval, reaching an asymptote at about $400 \mathrm{~ms}$, but were already detectable after $50 \mathrm{~ms}$ using a change detection paradigm. Diedrichsen could also show that distortional effects depended on encoding the target relative to the landmarks before the retention interval, suggesting that the landmarks affected the encoding of the target and that they had to be part of the memory representation for spatial distortions to occur.

\subsubsection{Models of spatial memory distortions}

The Nelson-Chaiklin Model. Nelson and Chaiklin (1980) proposed a weighted distortion theory to account for the data patterns obtained in their experiments. In their view, the remembered target position is distorted with respect to the 
position of all landmark stimuli in the visual field. Distortion is supposed to be toward the nearest landmark, with the magnitude of distortion increasing for positions more distant from the landmark. This prediction is derived from knowledge of the psychophysical function for remembered distance, which has a Steven's exponent below 1 (Gescheider, 1997) and therefore leads to an underestimation of target-to-landmark distance which increases with distance. In a situation with multiple landmarks, each landmark contributes to the overall pattern depending on its distance from the target, with larger weights for closer landmarks. Therefore, a target close to one landmark but far from all other landmarks is influenced almost exclusively by the nearest landmark. In general, however, the effects of multiple landmarks on a target will partly oppose each other, leading to so-called "equilibrium points" where all effects cancel out and no distortion can occur. Note that because of Nelson and Chaiklin's use of circular and elliptical displays, the term "landmark" acquires a more general meaning here and does no longer refer to a single visual object (like one of the small circles in Diedrichsen's experiments), but to all the points making up the large circle or ellipse enclosing the targets. Therefore, the distorting effects of such a stimulus on a single target must be derived by mathematical integration (i.e., by forming the path integral) over all "landmark" points comprising the circle.

Unfortunately, there is no concrete mathematical formulation of the theory, and this lack of formalism leaves the theory somewhat unclear in important points. One issue is how the opposing effects of the flattening psychophysical function and the decreasing weighting function actually determine the amount and direction of distortion. Consider the case where the weighting function is constant and does not depend on distance from the landmark. Then, all landmarks would simply add, and their effects would superimpose, at least up to a scaling constant. Each landmark, no matter how far from the target, would contribute to the net distortional effect. In contrast, with a weighting function steeply decreasing with landmark distance, farther landmarks would have little or no effect on a target, and distortional effects of a landmark would be locally restricted (see Exp. 5 and 6 below for an evaluation of these two possibilities). Such a restriction would in turn oppose the distortional effects of the psychophysical function, which can become large only at some distance from the landmark. Predictions from such a model are far from clear unless quantitative assertions are formulated. However, one clear prediction is that distortions near the 
landmarks should be small and that larger distortions can occur only at some distance from the landmarks.

Another problem is that the model is deterministic rather than stochastic. This is problematic because the authors regard the circular stimuli they use as consisting of an infinity of small landmarks, all independently influencing the target point. Such a model makes clear predictions only as long as landmarks induce a bias of target location but not alter the variance associated with it. However, if each landmark also contributes some increase in positional uncertainty of the target, the variance of the remembered target position must increase, and the final "equilibrium" state of the target must be quite unstable. In its current form, the model does not predict any particular pattern of variances. We will see that variance considerations are critical for understanding the occurence of spatial memory distortions (e.g., Exps. 1 and 2).

The Huttenlocher et al. Model. In contrast to Nelson and Chaiklin's (1980) proposal, Huttenlocher, Hedges, and Duncan (1991) suggest a stochastic prototype model where spatial memory distortions are explained by high-level cognitive processes involved in segmenting the visual stimulus. It is assumed that participants' responses are not only influenced by their recollection of the target location but also by the spatial "category" it is assigned to. Thus, there are two levels of representation in spatial memory: a fine-grained map of spatial locations and a coarse scheme of spatial regions and boundaries between regions where little more than topological relations are encoded. Although there is some uncertainty associated with both of these representations - remembered location and spatial categories -, they are both unbiased.

What forms a spatial "category" does not strictly depend on stimulus geometry. Instead, it must be determined indirectly from the distribution of target reproduction attempts. In Huttenlocher et al.'s (1991) experiments, participants had to reproduce target dots presented within a large circle. It was found that reproductions were not scattered uniformly across the circular area but that the horizontal and vertical meridians were systematically avoided. Thus, the authors concluded that participants spontaneously segmented the circle into four quadrants, which furthermore served as spatial categories. In principle, the layout of the categories must be empirically determined for each new stimulus configuration and may be determined by grouping processes, symmetry considerations, or simply subjective preferences of the participants. Because the model equations can be applied only to one coordinate at a time, 
it is crucial that the coordinate system chosen by the participants (e.g., polar coordinates centered on the circle, cartesian coordinates, or anything else) can also be determined post hoc.

In Huttenlocher et al.'s (1991) model, distortions in spatial memory can arise when category information is used to assist in the reconstruction of an imprecisely remembered target location. There are two processes biasing such responses towards the category prototype. First, because the fine-grained locational information is uncertain (modelled by a Gaussian distribution), it is possible that a target is accidentally remembered in the wrong spatial category. Correct memory for category membership of the target is then used to discard these cases, leading to truncation of those parts of the memory distribution that overlap category boundaries. As a result, the distribution skews away from category boundaries. However, these truncation biases are quite moderate and quickly dissipate with distance from the boundary.

Second and more important, distortions arise from a sort of guessing process where category membership is used to partly replace an imprecisely remembered target location. When memory for location is very uncertain, the prototype of the category is remembered instead, which is assumed to be located somewhere in the "center" of each category (empirically determined from the scatter of responses). In contrast, with very certain location information, the prototype need not be used; the relative weights of locational and category information therefore depend on the amount of locational uncertainty. Note that this process also biases remembered target positions away from category boundaries, towards the prototypes of the categories, again by combining two undistorted representations.

In contrast to Nelson and Chaiklin's (1980) model, Huttenlocher et al.'s (1992) model makes predictions about the pattern of variances. Because prototypes tend to attract and boundaries tend to repulse remembered targets, the boundary must be a region of high positional uncertainty and large variance in responses, whereas the center of the category must have low uncertainty and small variance. Put differently, targets located on the boundary are in a state of unstable equilibrium where opposing forces are trying to draw the representation in different directions but cancel each other so that memory remains unbiased. In contrast, targets located at the prototype location are in a stable equilibrium because forces from all directions are pointing towards this location. 
Nelson and Chaiklin's (1980) weighted distortion theory and Huttenlocher et al.'s (1991) prototype theory differ in several important respects. Nelson and Chaiklin regard spatial memory distortions as an inadvertible property of the cognitive system that only depends on the geometry of landmark stimuli, whereas Huttenlocher et al. point out the importance of cognitive strategies in encoding spatial layout. Prototype theory therefore allows for an impact of spatial reference systems other than those explicitly given by landmark geometry, whereas weighted-distortion theory does not. In Nelson and Chaiklin's model, the effects of new stimulus configurations can be predicted by combining the individual effects of all "landmark" elements comprising them (although it is not clear whether these effects are locally restricted), whereas in Huttenlocher's model categories can only be inferred post hoc. Huttenlocher et al.'s model predicts that target reproductions are biased away from landmarks and category boundaries and that these effects are strongest near these boundaries; in contrast, Nelson and Chaiklin's model supposes that distortion is towards the landmark and that the effect becomes larger as the distance from the landmark increases. Finally, weighted-distortion theory makes no predictions about regions of high or low positional certainty because it is inherently deterministic, whereas prototype theory predicts large variances at category boundaries and low variances at prototype locations.

Attentional distortions in topographical maps. Cells within topographical maps are not independent: most of them display some center-surround type of receptive field which strongly suggests mutual inhibitory connections between adjacent cells. Therefore, cells are highly malleable and principally able to mutually influence their spatial coding properties (Gilbert, 1998). Today, such dynamic properties of topographical networks are widely regarded as forming the basis of spatially selective attention (e.g., Desimone \& Duncan, 1995).

Suzuki and Cavanagh (1997) have shown how attentional manipulations might lead to distortions in topographical visual maps. They presented participants with a Vernier stimulus where two vertical bars that were vertically aligned were flashed on the screen. It was found that when a distractor stimulus was presented next to the upper bar, the bar appeared shifted away from the distractor, and the two bars appeared misaligned. The effect was larger when a second distractor was introduced that biased the lower Vernier bar into the other direction. In one crucial experiment, the authors presented the two Vernier bars with four distractors, two to the sides of 
the upper bar and two to the sides of the lower bar. They manipulated visual attention by introducing a secondary task, flashing a probe stimulus that could only appear in some of the distractors. It was found that Vernier bars appeared shifted away from attended distractors but not from unattended distractors. Other work corroborates the idea that shifts of attention leads to perceptual distortions. For example, visual space undergoes large compression just prior to eye movements (Ross, Morrone, \& Burr, 1997), which are normally preceded by visual attention shifting towards the target position.

Suzuki and Cavanagh (1997) proposed several mechanisms how this perceptual distortion might occur, assuming that the positions of the stimuli were coded in a topographic array of visual cells. First, cells in the focus of attention might narrow their spatial tuning curves, providing higher spatial resolution for the attended region (Moran \& Desimone, 1985; Spitzer, Desimone, \& Moran, 1988). Second, receptive fields might change their position, shifting towards the focus of attention (Connor, Gallant, \& Van Essen, 1994). Third, cells in the focus of attention might inhibit those outside the focus. It can be shown that all these mechanisms would lead to bias in a population-coded representation of the Vernier stimuli, so that the bars would appear shifted away from the attended distractors. Similar effects might be expected for spatial memory representations because dIPFC cells coding space in short-term memory are also topographically organized. Thus, Suzuki and Cavanagh's (1997) approach represents an interesting alternative to the theories by Nelson and Chaiklin (1980) and Huttenlocher et al. (1991).

\subsubsection{Overview of the experiments}

In this thesis, nine experiments are reported. Experiments 1 and 2 center around the issue of how single visual landmarks locally reduce positional uncertainty but introduce systematic spatial biases in the process, which is predicted by Huttenlocher et al.'s (1991) model but not by Nelson and Chaiklin's (1980) model. Experiment 3 employs a two-landmark configuration to test both models' assumption that distortional fields should be independent of stimulus orientation. Experiment 4 investigates the time course of spatial memory distortions in a discrimination task allowing for very short masking intervals, showing that the effect builds up more quickly than previously estimated. Experiments 5 and 6 provide a critical test of Nelson and Chaiklin's assumption that distortional fields of arbitrary landmark configurations can 
be predicted from the fields of individual landmarks, showing that although the field of two landmarks can be locally predicted by the distortional fields of single landmarks, single-landmark fields do not combine linearly. Instead, landmarks produce a partition of visual space. Importantly, the results also serve to falsify Huttenlocher et al.'s prediction that response variances should be large at category boundaries and small at prototype locations. Finally, Experiments 7 to 9 show that extravisual spatial reference information influences distortional fields, thus falsifying Nelson and Chaiklin's assumption that distortion can be predicted from the visual stimulus alone. In sum, it is shown that neither model can account for all of the present findings, and that both models are wrong in important respects. An alternative model of spatial memory distortions (Trommershäuser, 2001) is discussed which relies on attentional preactivation effects in topographical cortical representations. 


\section{Part I: One Landmark}

\subsection{Experiment 1}

In previous experiments by Huttenlocher, Hedges, and Duncan (1991) and Laeng, Peters, and McCabe (1998), participants had to reproduce the exact location of a target with respect to a large circle which served as a spatial reference. This is visually quite different from the experiments by Diedrichsen (1998), where two small circles were used as landmarks. Experiments 1 and 2 explore the effects that single landmarks have on distortions in visual short-term memory.

One crucial assumption in Huttenlocher et. al.'s (1991) model is that there is a tradeoff between the exact memorized position of a visual target and the spatial certainty associated with it: in other words, between the amount of spatial bias (systematic error) of the memory representation and the variance (variable error) associated with it In their model, category membership is used to restrict the spatial distribution of possible remembered positions of a target by truncation at category boundaries and by weighting with the category prototype. This reduces the variance of remembered target positions but leads to spatial biases towards the center of the category. The idea is that a small amount of bias is traded in for a large reduction in positional uncertainty, which might lead to an overall improvement of coding accuracy.

To illustrate this, consider a target being presented either by itself or next to a long line. If the target is presented alone, there is an undisturbed distribution of possible target locations in visual memory, which might be approximated by a symmetric bivariate Gaussian distribution with expectancy equal to the correct target position and homogenous variance in all directions. With the target presented next to a line, however, participants will tend to use the line as a spatial reference, and will be unlikely to remember the target on the wrong side of the line. The part of the Gaussian overlapping the line will therefore be discarded ("truncated", in Huttenlocher's model, but other mechanisms might be conceived of as well). Although the variance of the remaining distribution is of course reduced, the expectancy is also shifted away from the truncation line, and more so the more the distribution overlaps the line. In this

\footnotetext{
1 I will use the terms "bias" and "systematic error" as well as the terms "variance" and "variable error" interchangeably.
} 
way, reducing variance by taking into account the visual landmarks will generally lead to biased memory.

Nelson and Chaiklin's (1980) model does not predict a tradeoff between positional uncertainty and bias; in fact, it makes no prediction at all about variable error. In their model, memory distortions arise because the distance between the target and the nearest landmark is underestimated. Therefore, the model predicts that bias should be small near a landmark. This is the opposite of what Huttenlocher et al.'s (1991) model predicts, because truncation bias should be largest near a truncation boundary.

Experiment 1 was designed to test these ideas directly. In this experiment, participants had to reproduce the position of a target on the horizontal or vertical meridian of the monitor screen. Additionally, circles of different radii were presented around the screen center which could serve as visual landmarks. It was expected that target positions near the landmark line would be distorted away from it while the variance associated with these targets would be smaller than for targets further away from the landmark. Of the three circles used, two were of sufficiently small radius to lie within the distribution of possible targets. The third circle was considerably larger so that its circumference line was well outside the region where targets could appear, thus serving as a baseline condition.

\subsubsection{Method}

\section{Participants.}

Eight students and staff members of the Institute of Psychology at the University of Göttingen (age 20 to 34, all female and right-handed) participated for course credits or for a payment of 12 to 15,- DM per hour. Their vision was normal or corrected-to-normal. One of them replaced an earlier participant whose data had to be excluded from analysis because of extremely low response precision.

\section{Apparatus.}

The experiment was controlled by a Personal Computer with an AMD K-2 processor $(300 \mathrm{MHz}$ ). Stimuli were presented on a 14" VGA color monitor (640 by 480 pixel $[\mathrm{px}]$ ) with a monitor retrace rate of $60 \mathrm{~Hz}$. Stimulus presentation was care- 
fully synchronized with the monitor retrace rate. Participants were seated at a distance of approximately $100 \mathrm{~cm}$ from the screen. Head position was not fixed.

a) Possible landmark and target locations.

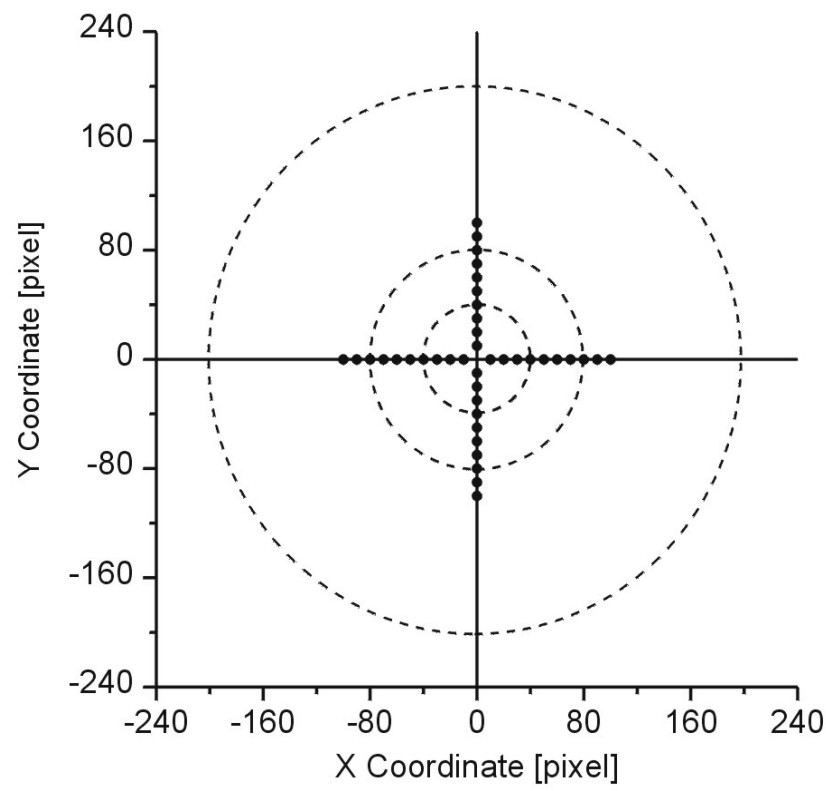

b) Time course of a trial.

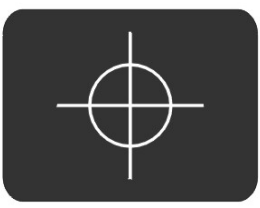

Meridians and landmark circle: $500 \mathrm{~ms}$

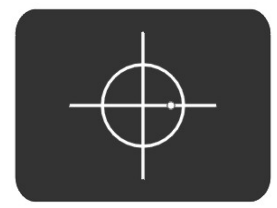

Target: $100 \mathrm{~ms}$

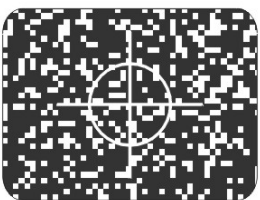

Dynamical mask (landmark still visible): $400 \mathrm{~ms}$

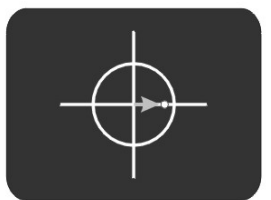

Reproduction of target location with mouse cursor

Fig. 1a) Stimulus configuration used in Experiment 1. Possible target locations are represented by black dots; possible landmark radii are represented by dotted circles. b) Time course of a trial.

\section{Stimuli.}

The horizontal and vertical meridians formed a green cross occupying the entire monitor screen (Fig. 1). Green landmark circles with a radius of $40 \mathrm{px}\left(1.03^{\circ}\right), 80$ px $\left(2.06^{\circ}\right)$, or 200 px $\left(4.75^{\circ}\right)$ were presented centered on the cross. The target was a small white dot $\left(0.09^{\circ}\right)$ that could appear on the meridian lines above, below, left, or right of the screen center. Possible target distances from the screen center ranged from $10 \mathrm{px}\left(0.26^{\circ}\right)$ to $100 \mathrm{px}\left(2.60^{\circ}\right)$ in steps of $10 \mathrm{px}$. For the small and medium 
landmark circles, this included targets that lay on the intersection of the circle with the meridian line. The mouse cursor had the same appearance as the target. A dynamic pattern similar to static interference on a television screen was used as a visual mask that filled the rectangular frame. It consisted of randomly chosen black and white elements $(2 \times 2 \mathrm{px})$, with one quarter of the elements white at any given time. Four different random patterns were presented in succession for $33 \mathrm{~ms}$ each, after which the sequence repeated itself.

\section{Procedure.}

At the beginning of each trial, the two meridian lines were presented together with the landmark circle. Participants were instructed to fixate on the crossing of the meridian lines and not move their eyes until the mouse cursor had appeared. After $500 \mathrm{~ms}$, the target was presented for $100 \mathrm{~ms}$, followed by the dynamic mask for 400 ms. Meridian lines and landmark circle remained visible during the masking interval. After the masking interval, the mouse cursor appeared in the screen center. Because the cursor could only be moved along the meridian where the target had been presented, the intersection of the circle with the meridian line can be regarded as a single landmark on a one-dimensional continuum. Participants used the computer mouse with their right hand to reproduce the location of the target and press the left mouse button when they had reached the remembered position. The button press elicited a $100 \mathrm{~ms}, 1000 \mathrm{~Hz}$ tone for feedback that the response had been registered. Participants were instructed to work quickly, but it was emphasized that accuracy rather than speed was important in this task. There was no feedback about the precision of the response.

Stimulus conditions were counterbalanced such that each combination of landmark radius and target position occured quasi-randomly and equiprobably, with each combination appearing once every four blocks. Each participant took part in two sessions of 20 blocks with 30 trials each. Each session started with an additional practice block of 30 trials with stimuli drawn randomly from the experimental blocks. Practice trials were not analyzed. At the end of the second session, participants were informed about the purpose of the experiment. 
Data treatment.

Trials with reponse times faster than $100 \mathrm{~ms}$ or slower than $10,000 \mathrm{~ms}$ were excluded because they likely reflected anticipatory or guessing behavior. Trials with a euclidean target deviation larger than $30 \mathrm{px}$ were also excluded. This procedure eliminated $1.66 \%$ of the raw data.

\subsubsection{Results}

Data were submitted to an analysis of variance (ANOVA) model that included target-to-center distance and landmark radius as independent variables. Here and in all subsequent ANOVA models reported in this thesis, repeated measures were performed on the trimmed raw data by introducing participants as an additional random factor (Maxwell \& Delaney, 2000). The $\alpha$ level was set to .05 for omnibus tests; separate error terms and Bonferroni $\alpha$ adjustments were used for planned comparisons and contrasts.

Fig. 2 shows the pattern of spatial distortions separately for the left, right, upper, and lower arm of the stimulus cross. In all landmark conditions, participants tended to place their reproductions too far away from the stimulus center. This effect was most pronounced near the center and on the lower arm of the stimulus cross. With the landmark radius lying within the range of possible targets, distortion within the circle was slightly reduced, but was fully present outside the circle. Again, this effect is present mostly on the lower arm of the stimulus cross. Unfortunately, ANOVA pooled across different arms of the stimulus cross (Fig. 3) revealed that participants behaved very inhomogenously so that neither the main effect of target distance, $F(9,63)=1.290, M S E=472.418, p>.25$, nor the main effect of landmark radius, $F(2,14)<1$, nor the interaction, $F(18,126)=1.133$, MSE $=468.895, p>.30$, became significant. 

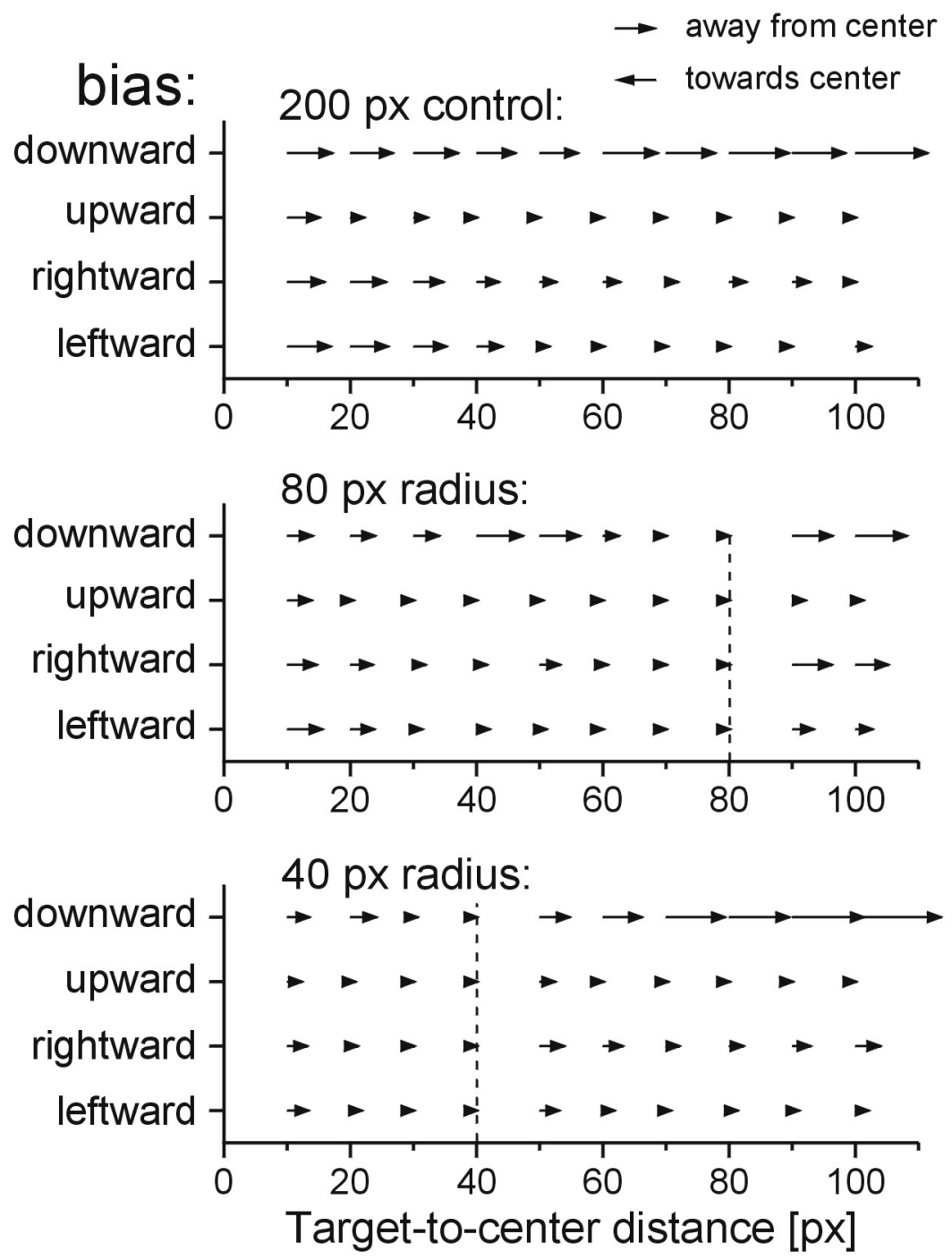

Fig. 2: Systematic error in the different landmark conditions of Experiment 1, separately for the lower, upper, right, and left arms of the stimulus cross. Arrows point from the veridical to the reproduced target location. Arrow length represents the amount of distortion and is magnified by a factor of 1.5 for readability. Dotted lines indicate the position of the 40-px and 80-px landmark circles.

To salvage at least some of the information in the data, the analysis was repeated with participants as a fixed rather than a random factor. [This analysis showed that at least within the sample of people tested, remembered target locations were biased away from the landmark lines, mainly towards the outside. The main effect of target distance was now significant, $F(9,9201)=16.218, M S E=37.574, p<.001$, as

\footnotetext{
${ }^{2}$ Fixing the participant factor allows for generalizations only within the current sample of participants but not beyond the sample (Cronbach, Gleser, Nanda, \& Rajaratnam, 1972), therefore trading in generalizability for statistical power.
} 
was the main effect of landmark radius, $F(2,9201)=15.629, M S E=37.574, p<$ .001 , with the $40 \mathrm{px}$ radius producing the smallest and the $200 \mathrm{px}$ radius producing the largest deviations from the true target position. Most important, the interaction of both factors was also significant, $F(18,9201)=14.144, M S E=37.574, p<.001$. Finally, planned contrasts showed that target positions presented within each landmark circle were remembered with less error than the same target positions presented within the 200-px control radius, $F(1,1902)=261.668, M S E=13.006, p<.001$ for the 40 px radius, and $F(1,4449)=63.604, M S E=33.485, p<.001$ for the 80 px radius.

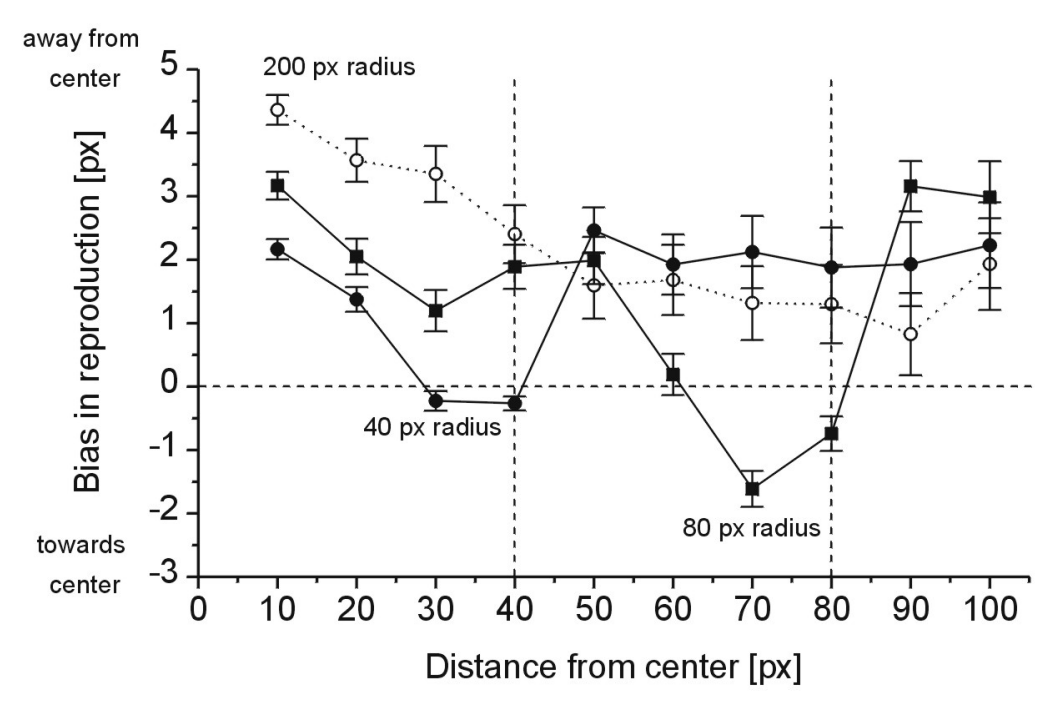

Fig. 3: Systematic (signed) error pooled across different arms of the stimulus cross as a function of target-to-center distance and landmark condition. Vertical dotted lines indicate the location of the smaller landmark circles. Note that standard errors are between trials, not subjects.

Participants appear more homogenous when euclidean distance from the true target position (i.e., unsigned instead of signed error) is used as a dependent measure, so that fixing the participant factor is unnecessary. This measure takes into account the variance of responses as well as their spatial bias. Fig. 4 shows that when the landmark circle is much larger than the range of possible target locations, euclidean error increases with distance from the center, $F(9,63)=6.405, M S E=175.413$, $p<.001$. Planned comparisons show that the increase is linear, this trend being the only significant polynomial contrast, $p<.001$ (quadratic and cubic trends: $p>.4$ and .2 , respectively). However, with smaller radii of landmark circles, euclidean error near 
the circle is lowered dramatically, leading not only to a main effect of target-to-center distance, $F(9,63)=26.581, M S E=133.109, p<.001$, but, more importantly, to a main effect of landmark radius, $F(2,14)=26.290, M S E=219.788, p<.001$, and an interaction of target distance with landmark radius, $F(18,126)=5.702, M S E=$ 158.237, $p<.001$. This is also reflected in significant linear, quadratic, and cubic trends for both radii (all $p<.001$ ). Most participants showed similar patterns. The pattern of euclidean error closely follows the pattern of response variances, so that regions of small euclidean error can also be regarded as regions of high positional certainty and low variable error.

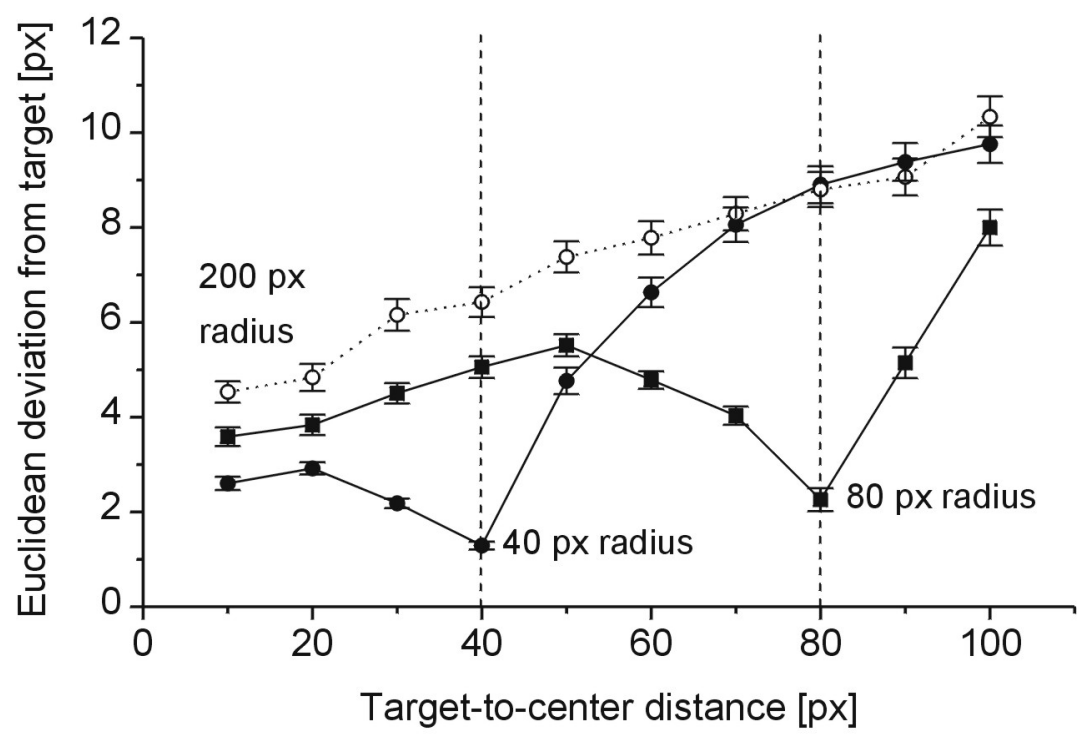

Fig. 4: Euclidean (unsigned) deviation from the target as a function of target-to-center distance and landmark condition. Standard errors are between trials.

\subsubsection{Discussion}

Compared to a control condition, the presentation of circles as landmark stimuli enhances memory for target locations. In the control condition where the circle is so large that it cannot be efficiently used as a spatial reference, there is some bias away from the display center that levels off with target distance. There is an especially pronounced downward bias along the lower arm of the stimulus cross. For targets within the 40-px and 80-px circles, bias is reduced, but is reinstated for targets outside these circles. However, these findings are plagued by massive differences 
between participants. Regarding the unsigned (euclidean) rather than the signed errors reveals a much clearer picture, indicating that euclidean error increases linearly with distance from the display center in the control condition, but is greatly reduced in the vicinity of the landmark circles. This pattern is also consistent across participants. Combining information from spatial bias and variance of responses, euclidean error essentially reflects the pattern of variances.

Huttenlocher et al.'s (1991) model suggests that variance can be diminished at category boundaries only at the expense of newly introduced spatial biases: because the landmark line cuts off part of the memory distribution that would normally arise, the expectancy of the distribution is shifted away from the landmark. Unfortunately, the data concerning spatial biases in the present experiment must be regarded with caution and cannot be generalized beyond the present sample of participants. However, at least in the participants studied, the data are consistent with Huttenlocher's prediction in that reproductions are distorted away from the perimeter of the landmark circles. The effect is not symmetric, though, because there is only distortion towards the outside of the circle, not towards the inside.

Regarding the pattern of variances, the data also support Huttenlocher et al's (1991) assumption that visual landmarks create local regions of high positional certainty, massively reducing the variance of responses in the vicinity of the landmarks. However, this seems to be only a local process because the pattern of euclidean error suggests not only that errors reoccur outside the landmark circle, but even that these errors reach the size of those in the control condition. This is surprising because the variance might be expected to increase linearly with distance from the landmark instead of distance from the center now, like in a renewal process. Instead, variance seems suppressed within the landmark circle but not at some distance from the landmark circle. This pattern is consistent with evidence from attentional research suggesting that a "spotlight" of attention can be directed to contiguous spatial regions of varying size (Eriksen \& Eriksen, 1974; LaBerge \& Brown, 1989; LaBerge, 1995). Many studies have shown that targets within the spotlight are detected and identified both faster and with higher accuracy than targets outside the spotlight (e.g., Downing, 1988). Possibly, large circular landmark stimuli as used here capture and calibrate the spatial distribution of attention, setting both its location and size. Moreover, the asymmetrical pattern of systematic error (Fig. 3) at the landmark boundary might 
suggest a spotlight explanation, with spatial biases only directed away from the spotlight, but not towards the inside of the spotlight.

Finally, the data directly contradict Nelson and Chaiklin's (1980) assumption that the amount of spatial bias is small near the landmark and increases with distance from the landmark. However, the model's employment of a monotonously decreasing weighting function might suffice to predict spatial bias gradually decreasing with distance. However, even with such a weighting function, bias should be smallest rather than largest near a landmark, a prediction clearly falsified by the data. Thus, the evocation of a psychophysical function for memorized distance seems not sufficient for explaining the pattern of spatial memory distortions.

\subsection{Experiment 2}

Experiment 1 suggested that in reproducing the exact location of a visual target, additional visual stimuli are used as a spatial reference. This leads to higher accuracy of responses in terms of variable error, but also tends to introduce spatial biases so that reproductions are shifted away from the landmark stimuli.

Experiment 1 used displays where targets occured on the arms of a visible cross and landmarks were large circles providing spatial reference by intersection with the cross. Also, reproductions could occur only along the crosslines, allowing only for one-dimensional movements. These stimuli are quite dissimilar from the ones used by Diedrichsen (1998) where possible target locations were scattered across the display, the mouse cursor could be moved freely in two-dimensional space, and spatial reference was provided by two small landmark dots. Experiment 2 was conducted to explore the effect of a single landmark dot on spatial memory in a variant of Diedrichsen's paradigm where either the left or the right landmark was present, compared to a condition where no landmark was provided. As before, it was expected that landmarks would lower the variance of reproductions but introduce spatial biases leading to a distortion of visual memory. While Huttenlocher et al.'s (1991) model predicts that this effect should be large near the landmark, Nelson and Chaiklin's (1980) model predicts that it can be large only at some distance from the landmark.

A second purpose of this experiment was to check whether the effect of the dynamical pattern mask used in Experiment 1 could also be obtained with a simple 
brightness mask. There were several reasons to investigate this factor. First, it is known from the literature on visual masking that brightness masking implies more peripheral stations of the visual system than pattern masking (Breitmeyer, 1984). For example, brightness masking only works if test stimulus and masking stimulus are presented to the same eye, whereas a pattern mask can also be presented to the other eye (Turvey, 1973). Because combination of visual signals from the two eyes does not occur before area V1, cortical processing must be involved in pattern masking, whereas brightness masking probably has a strong retinal component. Therefore, stronger memory distortions in the brightness mask condition would argue for a strong precortical contribution. Second, it might be that the visual complexity of the dynamic mask with its high-frequency spatial and temporal motion transients might be a necessary precondition to obtain visual masking strong enough to effectively suppress afterimages of the target.

\subsubsection{Method}

\section{Participants.}

Six students (age 22 to 31, three of them male, all right-handed) of the Institute of Psychology at the University of Göttingen participated for course credits or for a payment of $15,-$ DM per hour. Their vision was normal or corrected-to-normal.

\section{Stimuli.}

The apparatus was as described in Experiment 1. All stimuli were presented against a black background $\left(0.01 \mathrm{~cd} / \mathrm{m}^{2}\right)$. Landmarks were green unfilled circles $(28.7$ $\left.\mathrm{cd} / \mathrm{m}^{2}\right), 11 \mathrm{px}\left(0.31^{\circ}\right)$ in diameter and $80 \mathrm{px}\left(2.27^{\circ}\right)$ to the left or right of the center of the screen. The target was a small white dot $\left(43.0 \mathrm{~cd} / \mathrm{m}^{2}\right)$ with a diameter of $3 \mathrm{px}$, presented at one of the 64 possible locations shown in Fig. 5a. When a landmark was presented, the target was at one of the 32 possible locations from the same half of the display. A dynamical mask was used as described in Experiment 1. Additionally, a simple brightness mask was used where the entire screen turned white (43.0 $\left.\mathrm{cd} / \mathrm{m}^{2}\right)$. 
a) Possible landmark and target locations.

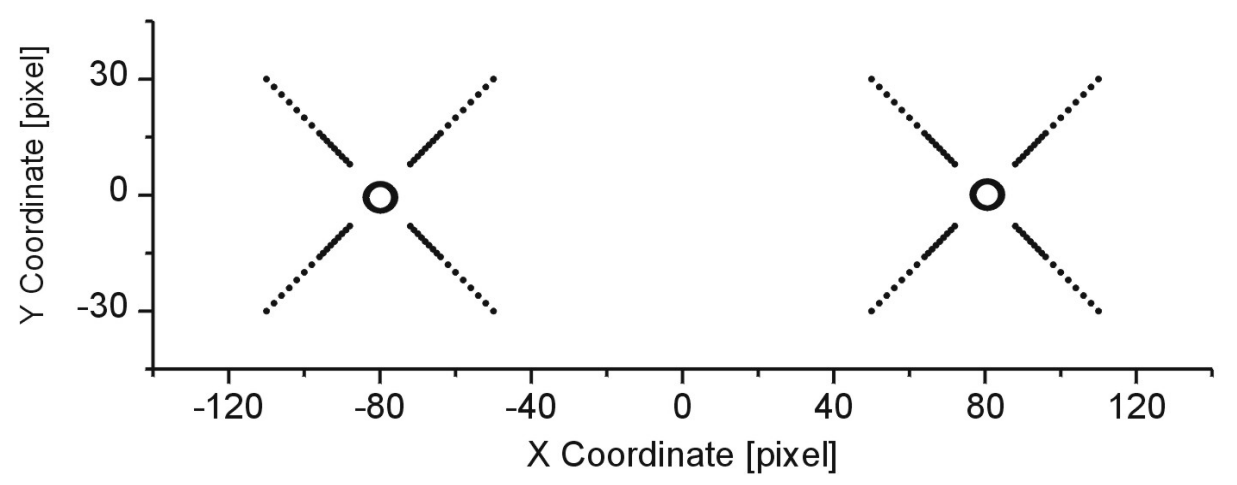

b) Time course of a trial.

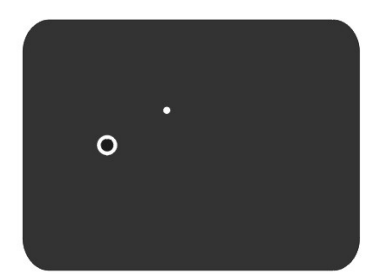

Target with one or two landmarks: $1500 \mathrm{~ms}$

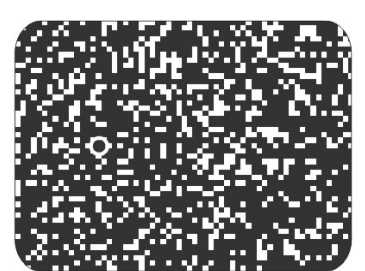

Dynamical mask (landmarks still visible): $500 \mathrm{~ms}$

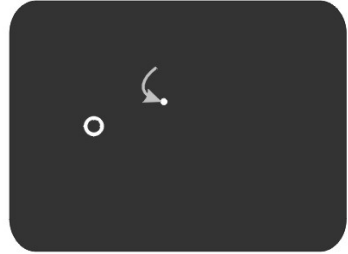

Reproduction of target location with mouse cursor

Fig. 5a: Possible landmark and target locations in Experiment 2. Note that either one landmark or no landmark was presented and that the target always appeared in the same display half as the landmark. $b$ : Time course of a trial involving the dynamical mask. The landmark also remained visible when a brightness mask was used.

\section{Procedure.}

A trial began with the appearance of the target together with either the left, right, or no landmark (Fig. 5b). Participants were allowed to inspect this stimulus for $1500 \mathrm{~ms}$ under free viewing conditions, without any restriction of eye movements. The target was then replaced by either the dynamic or brightness mask for $500 \mathrm{~ms}$ while the landmarks remained visible. Immediately after the masking interval, a mouse cursor looking exactly like the target appeared in the center of the screen. The participants' task was to use the mouse cursor to reproduce the target's location as exactly as possible and to press the left mouse button when finished. The button press elicited a $1000 \mathrm{~Hz}, 100 \mathrm{~ms}$ tone for feedback that the response had been registered. After an intertrial interval of 500 ms, a new trial began. Participants were instructed to work quickly, but it was emphasized that accuracy rather than speed was important in this task. 
Masking conditions were blocked such that dynamic and brightness masks were presented in alternating blocks, starting with the dynamic mask. The remaining stimulus conditions were counterbalanced such that each combination of landmark position (left, right, none) and target position occured quasi-randomly and equiprobably. Each combination appeared once per session. After each block, participants received summary feedback about their average euclidean deviation from the true target position, rounded to the nearest pixel.

Participants performed four sessions of 16 blocks with 32 trials each. Each session started with two additional practice blocks of 32 trials with stimuli drawn randomly from the experimental blocks, one block per masking condition. Practice trials were not analyzed. After the final session, participants were debriefed and received an explanation of the purpose of the experiment.

\section{Data treatment.}

Trials with reponse times faster than 100 ms or slower than 10.000 ms were excluded because these trials likely reflected anticipatory or guessing behavior. Trials with a euclidean target deviation larger than $30 \mathrm{px}$ or exceeding plus or minus three standard deviations were also excluded for each participant. This procedure eliminated $4.37 \%$ of the raw data.

\subsubsection{Results}

Fig. 6 shows the distribution of all reproductions performed in this experiment. When landmarks are present, responses cluster closely around the true target positions. Without landmarks, however, response variance is markedly increased, the clusters appear more fuzzy and are shifted away from the monitor center. It is necessary to distinguish between two types of spatial distortions here: biases that arise from local effects at the landmark and drift of the entire response distribution when no landmark is present. The type of visual masking used has no effect on response distributions. Because this factor did not show any effect in any of the preliminary analyses, it was dropped from the statistical design in subsequent analyses. 

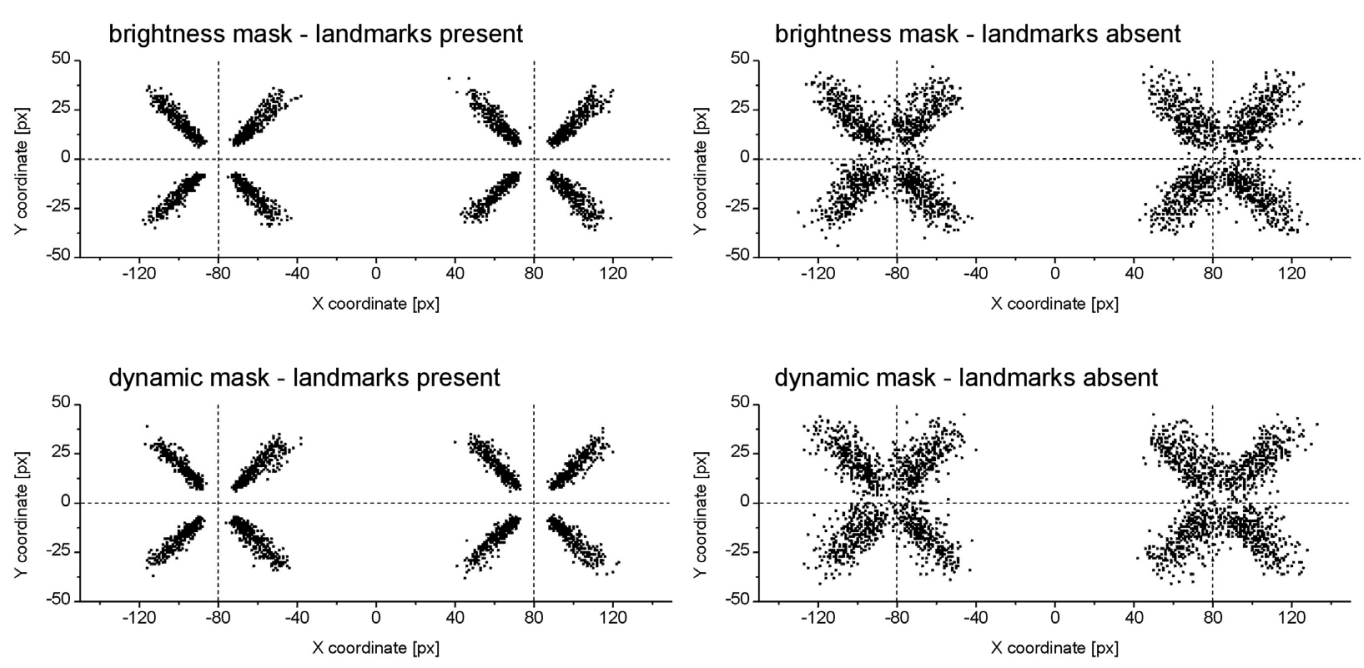

Fig. 6: Scattering of reproduced target positions as a function of landmark presence and mask type in Experiment 2. Results from targets in the left and right halves of the display are combined within each plot.

General effects in the distortional fields. Vectorial deviations of the average reproduced positions from the true target positions were partitioned into an angular and a radial component centered on the true position. Repeated-measures analyses of variance employing a random participant factor as described in Experiment 1 were performed on the (unsigned) radial error, with target-to-landmark distance and presence/absence of landmarks as independent variables. Left and right landmarks were pooled by reflecting all data from the right-landmark condition about the $y$ axis. Fig. 7 shows that radial error is dramatically reduced when a landmark is present, $F(1,5)=17.331, M S E=4235.478, p<.01$. There is also a main effect of target-tolandmark distance, $F(15,75)=7.975, M S E=15.250, p<.001$, suggesting that radial error tends to increase with distance from the landmark. However, this is only the case when the landmark is present, as reflected in a significant interaction, $F(15,75)$ $=6.823, M S E=13.009, p<.001$. Planned contrasts confirm that a linear trend is only detectable when landmarks are present, $p<.001$, but not when they are absent, $p>$ .190. As noted in Experiment 1, the radial (euclidean) error component reflects response variance as well as spatial biases. Thus, smaller radial error in the landmarks-present condition can be regarded as indicating reduced variable error. 


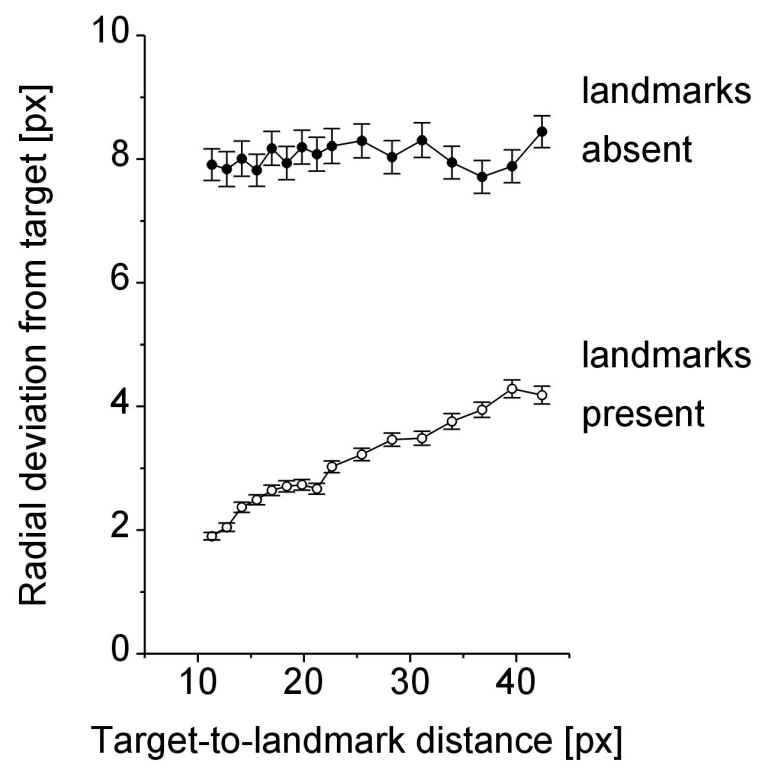

Fig. 7: The average radial component of distortions in Experiment 2 (i.e., unsigned euclidean error) as a function of target-to-landmark distance and landmark presence/absence. Standard errors are between trials.

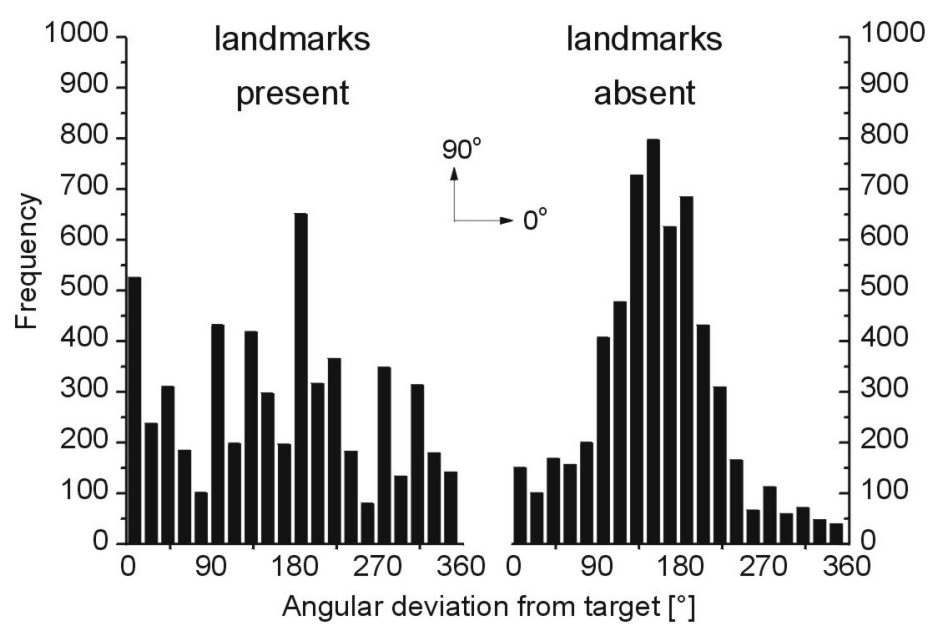

Fig. 8: Frequencies of the angular components of the distortions in Experiment 2. Note that data from the left and right halves of the display are pooled by mirror-reflection of the right half, such that the $0^{\circ}$ direction reflects deviation towards the inside of the display and $180^{\circ}$ reflects deviation towards the outside.

The angular component of response deviations had to be analyzed differently because it is not a straightforward problem to average angular data. Problems occur because the average direction of angles depends on the way the angles are coded. 
For example, numerically averaging two angles of $15^{\circ}$ and $345^{\circ}$ yields $180^{\circ}$, but recoding to $15^{\circ}$ and $-15^{\circ}$ yields an average of $0^{\circ}$. If it is known beforehand where the average direction should be (i.e., when all angles cluster closely around some center), this direction can be used as a reference, but when angles form a more diffuse distribution, the averaging problem must be based on vectorial methods and can be statistically analyzed only by referring to so-called wrapped distributions (see Fisher, 1993, for an introduction to those methods). Unfortunately, factorial approaches do not yet exist.

Therefore, angular effects were tested here by plotting the frequency histograms of angular deviations in all experimental trials separately for the conditions where landmarks were present or absent. Fig. 8 shows that with landmarks absent, the distribution of angles is clearly unimodal with a peak at about $160^{\circ}$, i.e., an outward and slight upward bias. A Kolmogorov-Smirnov test confirms that this distribution is significantly different from a rectangular distribution, $Z=20.198, p<.001$. In contrast, with landmarks present, there is no single peak in the distribution, so there is no clear bias in any particular direction. However, deviations in horizontal and, to a lesser degree, in vertical directions seem more frequent than deviations in other directions; in any event, this distribution also differs from a rectangular distribution, $Z$ = $9.058, p<.001$.

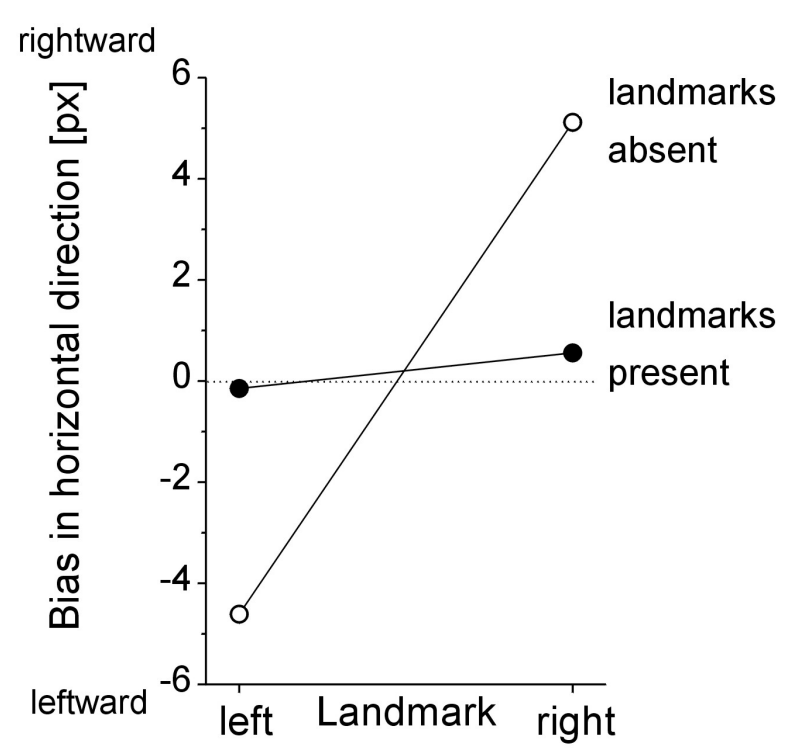

Fig. 9: Drift of the entire response distributions to the left or right of the display is absent when landmarks are presented. Standard errors (between trials) have been dropped because they would be smaller than the symbols. 
Drift of memory representations. The general outward bias in the condition without landmarks was tested in an additional ANOVA with horizontal deviation from the target as dependent variable and landmark side and presence/absence of landmarks as independent variables, the interaction of both factors (Fig. 9) confirming outward bias when landmarks are absent but not when they are present, $F(1,5)=$ 18.911, MSE $=3174.265, p<.01$.

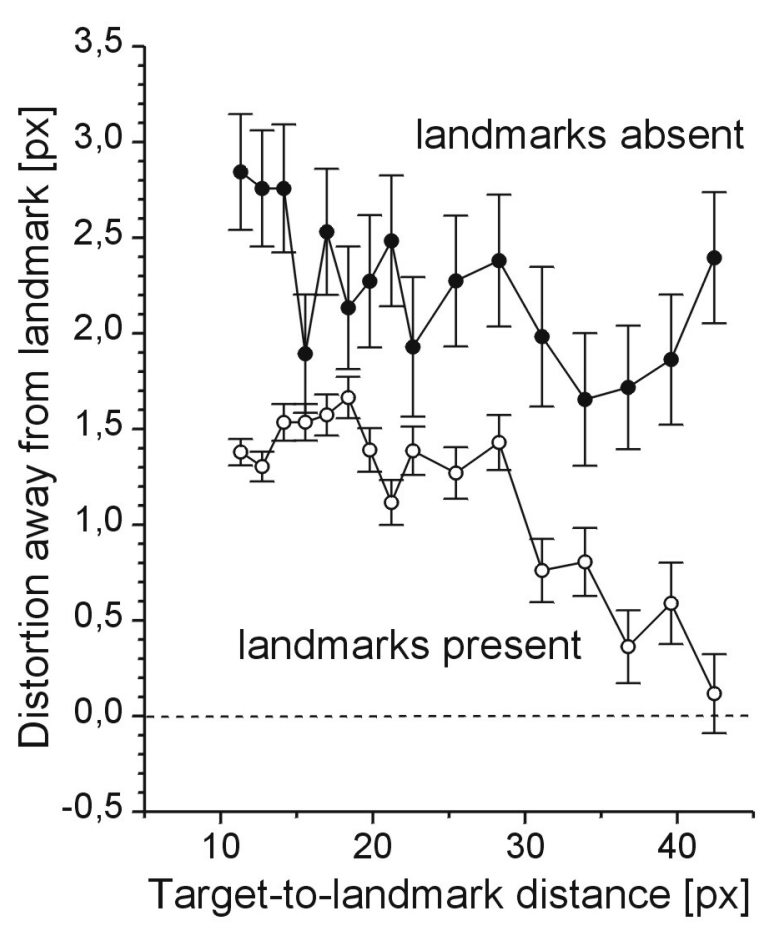

Fig. 10: Distortion away from the landmark as a function of target-to-landmark distance and presence/absence of landmarks. Standard errors are between trials.

Distortions at the landmark. In a final analysis, the effect of distortions near the landmark were analyzed by plotting the amount of distortion away from the landmark against target-to-landmark distance, pooled over left and right fields. Fig. 10 shows that this bias was stronger when landmarks were absent, although not significantly so, $F(1,5)=3.176, M S E=1119.237, p=.135$. Separate analyses for the landmark-present and landmark-absent conditions showed that in the landmark-absent condition, bias was nearly constant and only gradually diminishing with distance from the landmark, albeit with much random variation. Apparently, it was mainly determined by the entire response distribution's bias away from the display center. Accordingly, there was only a marginally significant effect of target-to-landmark 
distance, $F(15,75)=1.634, M S E=31.654, p=0.85$, which was associated with a significant (downward) linear trend $(p<.005)$ but no higher-order trends, all other $p>$ .20. In the landmark-present condition, bias was strongest at the target positions near the landmark and decreased with distance from the landmark until disappearing for those targets farthest away from the landmark. There was a significant effect of target distance, $F(15,75)=2.050, M S E=36.968, p<.05$, which was associated with both linear $(p<.001)$ and quadratic $(p<.001)$, but no higher-order trends.

\subsubsection{Discussion}

Presenting a single landmark in either the left or the right half of the display clearly leads to a change in the pattern of reproduced target locations. Most important, presentation of a landmark leads to markedly reduced scattering of reproduced target locations. This effect replicates findings from Experiment 1 and confirms the prediction that landmarks create regions of high positional certainty where the variance of memory representations is low. As before, the reduced variance also reduces the average reproduction error as measured by euclidean (unsigned) deviations from the target, a reduction by a factor of 4 near the landmark and still a factor of 2 farther away from the landmark (Fig. 7).

Does the reduced variable error of target reproductions come with a cost in terms of bias of remembered locations? This question is more difficult to evaluate, since in the absence of a landmark participants have difficulty remembering the exact locations of all targets, with the whole cluster of responses shifted away from the screen center. When landmarks are presented, this effect is completely abolished. Obviously (and not surprisingly), landmarks can serve as spatial anchorpoints that prevent a target location from drifting in spatial memory. A different issue is whether remembered locations within a cluster of responses are distorted. Fig. 10 shows that when the landmark is absent, targets tend to be remembered too far away from the position where a landmark can be presented, but this effect is hardly modulated by distance to the landmark location and only reflects the general bias away from the display center. With a landmark present, however, distortion away from the landmark is not only smaller but also clearly modulated by target-to-landmark distance: locations near the landmark are biased away from it, while the bias for locations far from the landmark approaches zero. As in Experiment 1, this effect is just the opposite of 
what is predicted by Nelson and Chaiklin (1980), namely that distortion should be smallest near the landmark and increase with distance.

The variation of different visual masks had no effect whatsoever, with employment of a brightness mask leading to exactly the same pattern of spatial distortions as a dynamic pattern mask. This finding is important for two reasons. First, the mask does not have to carry complex pattern and motion signals to be effective; it is not necessary to swamp the visual system with spatiotemporal noise for spatial distortions to develop. Second, it suggests that the relative amount of cortical and precortical processing during the masking interval has little influence on memory performance in the present task. For example, if the amount of spatial distortion had only depended on the disruption of a retinal after-image, a brightness mask might have been expected to compromise this after-image more strongly because it had a higher overall energy than the dynamic mask. On the other hand, if the effect of the dynamic mask had been mainly to interfere with a fine-grained spatiotemporal memory representation, the featureless brightness mask would have been expected to have no effect at all. Instead, equal efficiency of both mask types suggests that purely perceptual interference is not crucial for spatial distortions to occur. Instead, Diedrichsen (1998) has found that the duration of the masking interval is the decisive factor, with stronger distortion at longer masking intervals.

Together, Experiments 1 and 2 show that visual landmarks serve to reduce positional uncertainty in their vicinity. In the absence of landmarks, stimulus representations tend to drift in spatial memory. With landmarks present, spatial memory is distorted away from the landmark position; contrary to Nelson and Chaiklin's (1980) model, this distortion is largest near the landmark and levels off with distance. Huttenlocher et al.'s (1991) model is consistent with these results because it predicts that memory distributions are truncated at a visible landmark, leading to reduced variance as well as spatial bias away from the landmarks. 


\section{Part lla: Two Landmarks -}

\section{Orientational Invariance and the Time-Course of Distortion}

\subsection{Experiment 3}

In Experiments 1 and 2, only one landmark was present while participants encoded the location of the target. In previous experiments by Diedrichsen (1998) and Diedrichsen and Werner (submitted), two-landmark configurations were used with the landmarks located at some distance to the display midpoint. It was found that remembered target locations were biased away from the landmarks and (in some participants) also from the midpoint between the landmarks, forming a distortional field with obvious regularity and symmetry. Thus, with two landmarks instead of one, new geometrical properties are emerging, as landmarks define two axes of symmetry and one midpoint where these axes cross.

However, previous experiments exclusively used displays in horizontal or vertical orientations. This intrinsic stimulus orientation coincides with extrinsic reference systems that might possibly be used by the participants, e.g., the edges of the monitor, the direction of gravity, or the vertical body axis. It is therefore not clear what is responsible for the regularity and symmetry observed in these distortional fields: geometrical features of the stimulus configuration, possible extrinsic reference systems used by the participants, or the alignment of both. Note that theories claiming that distortional effects are exclusively determined by stimulus geometry (Nelson \& Chaiklin, 1980; Watson, 1977) predict strict independence from extrinsic reference systems, while models built upon cognitive strategies of image segmentation (Huttenlocher et al., 1991) at least allow for some effects of extrinsic references.

Experiment 3 was designed to assess whether misalignment of the intrinsic stimulus orientation with extrinsic frames of reference would change the pattern of distortion. A total of 13 target positions between the two landmarks was used where especially salient effects of distortion could be expected due to previous results. In one condition, the two landmarks were horizontally or vertically aligned, whereas in a second condition the whole configuration of landmarks and targets was rotated by plus or minus $45^{\circ}$, resulting in two diagonal landmark configurations. All stimuli were presented within a rectangular frame that was always aligned with the monitor's sides 
(and therefore also with the gravitational axis). As before, the task was to reproduce the exact location of a briefly presented target with respect to the two landmarks. A secondary purpose of this experiment was to measure spatial memory distortions for some critical target points that were to be used for estimation of the time course of memory distortions in Experiment 4.

\subsubsection{Method}

\section{Participants.}

Six undergraduate students (age 23 to 31 , all female, all right-handed) of the Institute of Psychology at the University of Göttingen participated for course credits or for a payment of 15,- DM per hour. Their vision was normal or corrected-to-normal.

a) Possible landmark and target locations.

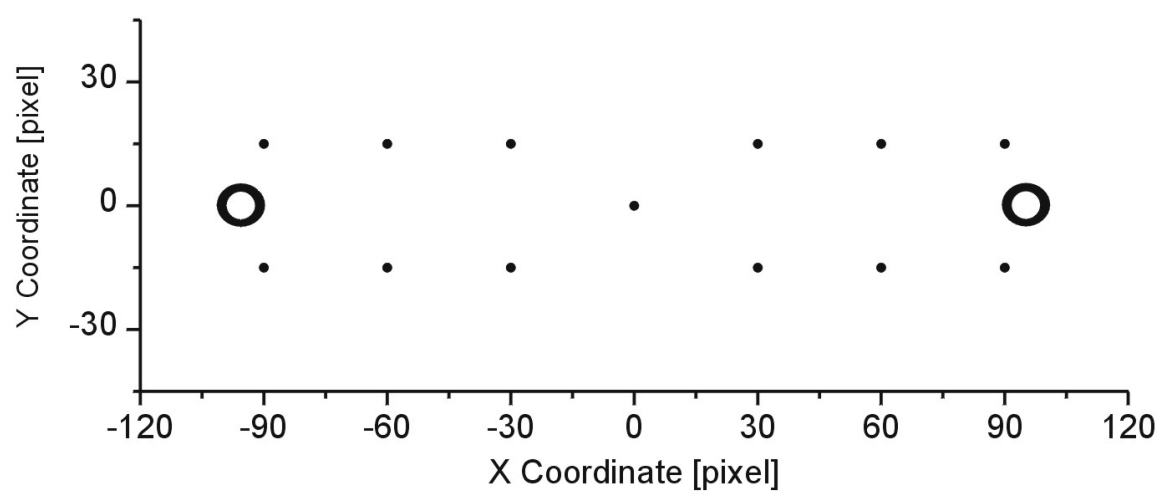

b) Time course of a trial.

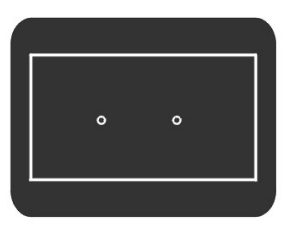

Landmarks: $500 \mathrm{~ms}$

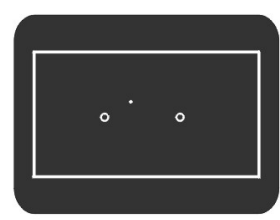

Target: $500 \mathrm{~ms}$

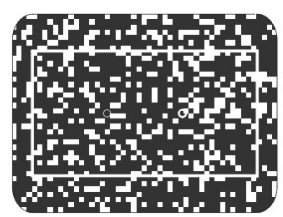

Dynamical mask (landmarks still visible): $400 \mathrm{~ms}$

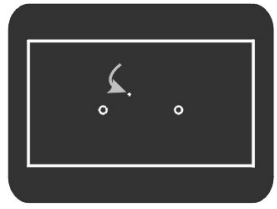

Reproduction of target location with mouse cursor

Fig. 11a: Landmarks and possible target locations in Experiment 3. b: Time course of a trial.

Apparatus and Stimuli.

The apparatus was the same as described in Experiment 1. All stimuli were presented within a white rectangular frame $\left(600 \times 380 \mathrm{px}, 17.16^{\circ} \times 10.86^{\circ}\right)$ at the 
center of the screen against a black background (Fig. 11). This frame was used to enhance possible effects of alignment with horizontal or vertical allocentric axes. Landmarks were two green unfilled circles, $9 \mathrm{px}\left(.26^{\circ}\right)$ in diameter and $100 \mathrm{px}\left(2.86^{\circ}\right)$ away from the center of the screen. The target was a small white dot with a diameter of $3 \mathrm{px}$, presented at one of 13 possible locations. Landmarks and target could appear in any of four orientations. In the situation where the two landmarks were horizontally aligned ( $0^{\circ}$ orientation), one of the possible locations was at screen center, the other 12 at $y$ coordinates of $\pm 15 \mathrm{px}$ and $x$ coordinates of $\pm 90, \pm 60$, and $\pm 30 \mathrm{px}$. For the $90^{\circ}$ orientation, the $x$ and $y$ coordinates were exchanged. The diagonally aligned conditions (plus and minus $45^{\circ}$ orientations) resulted from counterclockwise rotation of the two landmarks and the corresponding targets around the center of the display. The frame and mask remained unrotated. A dynamic mask as described in Experiment 1 was used.

\section{Procedure.}

Each trial began with the presentation of the two landmarks within the white frame. After $500 \mathrm{~ms}$, the target appeared at one of the five possible locations and remained on the screen for $500 \mathrm{~ms}$ before it was replaced by the dynamic mask for $400 \mathrm{~ms}$. The landmarks remained visible until the participant responded and were also visible during the masking interval. The participants' task was to use the mouse cursor (which looked exactly like the target) to reproduce the target's location as exactly as possible and to press the left mouse button when finished. The mouse cursor appeared randomly in the center of one of the landmarks to prevent the use of the initial cursor position as an additional spatial reference. The button press elicited a $1000 \mathrm{~Hz}, 100 \mathrm{~ms}$ tone. After an intertrial interval of $500 \mathrm{~ms}$, a new trial began. The instruction emphasized accuracy rather than speed.

Stimulus conditions were counterbalanced such that each combination of target position and stimulus orientation occured randomly and equiprobably, with each combination appearing once every four blocks. The center target position appeared twice as often as any other target position to yield equal numbers of observations for all $x$ and $y$ coordinates.

Each participant performed one session of 20 blocks with 28 trials each. The session started with an additional practice block of 28 trials. After each block, participants received summary feedback of their average euclidean deviation from the tar- 
get. After the session, participants were debriefed and received an explanation of the purpose of the experiment.

\section{Data treatment.}

For the following analyses, response times shorter than $100 \mathrm{~ms}$ and longer than $6000 \mathrm{~ms}$ were excluded. Also exluded were all trials where responses were more than $30 \mathrm{px}$ away from the original target or where the deviation was more than three standard deviations larger or smaller than the average of this participant. This procedure eliminated $3.24 \%$ of trials.

\subsubsection{Results}

Data were analysed in a repeated-measures MANOVA design with horizontal and vertical components of distortion as depended variables and target position and orientation (straight vs. oblique) as independent variables] Analogous to the ANOVA models described in Experiments 1 and 2, the repeated-measures model was implemented by including participants as an additional random factor (Maxwell \& Delaney, 2000). Wilk's $\Lambda$ was used as a test statistic. Horizontal and vertical orientations were collapsed after proper normalization, as were the plus and minus $45^{\circ}$ orientations, because different orientations within the straight or oblique conditions were not involved in any significant main effects or interactions, all $p>.07$.

Results will be reported as if all vector fields had been rotated to horizontal, referring to the axis of elongation as "horizontal" and to the perpendicular axis as "vertical". Fig. 12 shows that remembered target locations were distorted away from the virtual line connecting the landmarks, the midpoint between the landmarks, and the landmarks themselves. MANOVA confirmed a main effect of target position indicating that the vectors varied with target position, $\Lambda(24,118)<.0014 p<.001$. This effect was very strong in both the horizontal and vertical components of distortion, $F(12,60)=340.475$ and $2792.155, M S E=203.998$ and 335.077 , respectively, both $p$ $<.001$ (Fig. 13). Overall size of the effect did not depend on whether the stimulus orientation was straight or oblique, $\Lambda(2,4)=.491, p>.24$, but there was a significant

\footnotetext{
3 Readers unfamiliar with MANOVA may regard it as a straightforward extension of univariate analysis of variance, only that two-dimensional vectors rather than real numbers serve as the dependent variable (indeed, ANOVA is just a special case of MANOVA where the dependent variable is a one-dimensional vector).

${ }^{4}$ Note that smaller values of $\Lambda$ indicate larger effects.
} 
interaction of target position and stimulus orientation, $\Lambda(24,118)=.469, p<.003$. This was mainly due to the horizontal component of distortion having smaller values in the vicinity of the landmarks for the oblique conditions, $F(12,60)=2.293$, MSE = $38.454, p<.02$, but there was also a somewhat smaller effect in the vertical component of distortion in the oblique conditions, $F(12,60)=2.200, M S E=71.030, p<.03$.
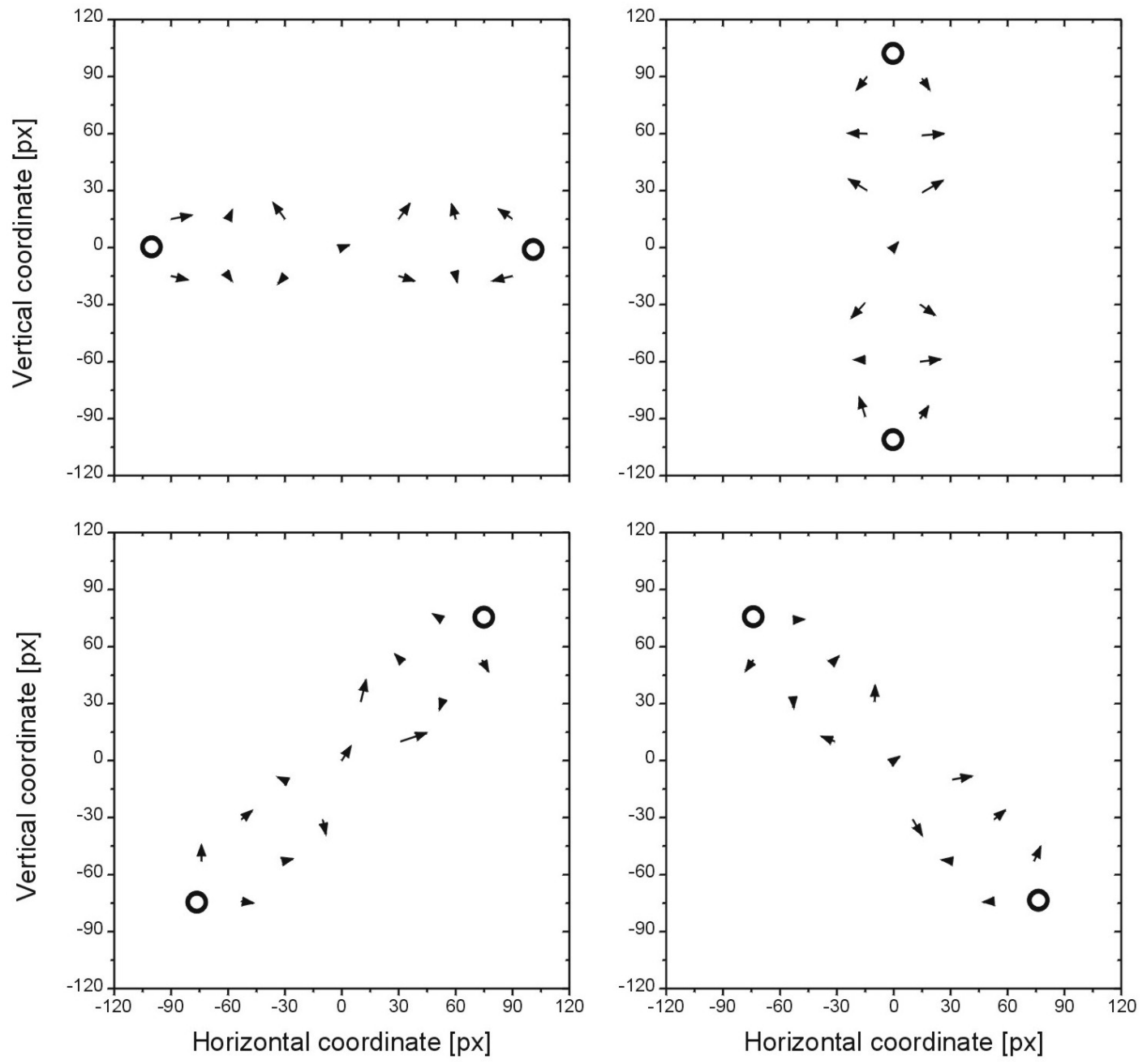

Fig. 12: Distortional fields from Experiment 3 as a function of orientation of the stimulus configuration. Arrows are magnified by a factor of 2 for readability. 

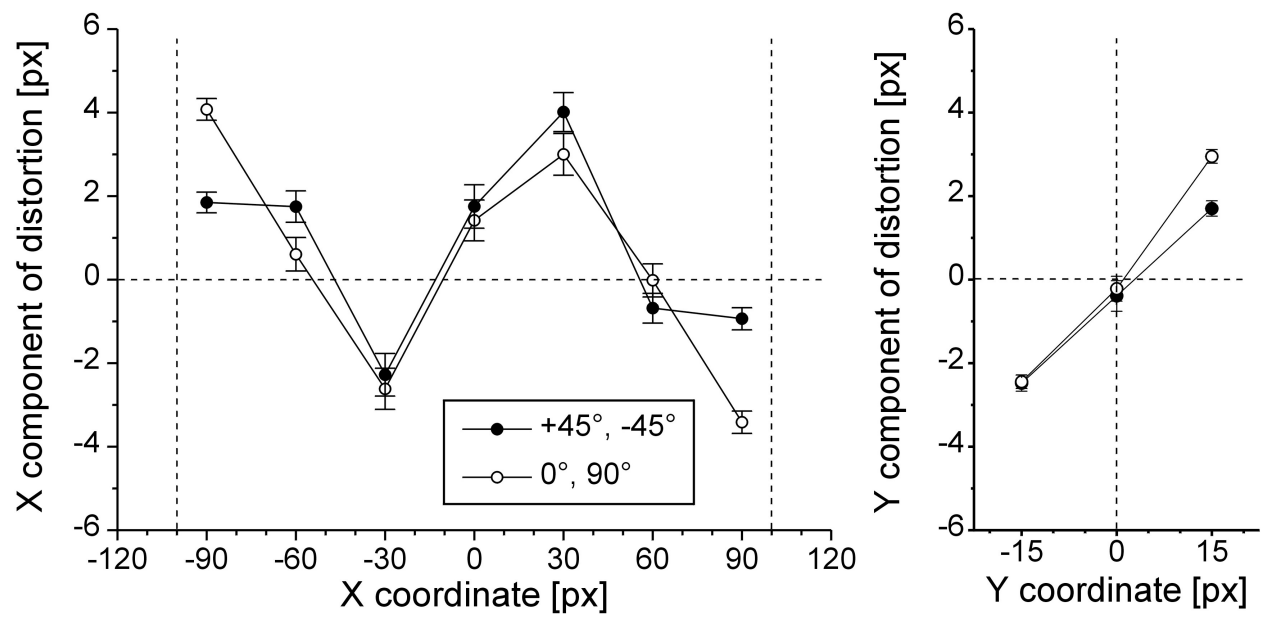

Fig. 13: Systematic error in reproduction as a function of horizontal (left) and vertical (right) target position, averaged across the remaining coordinate. Standard errors are between trials.

\subsubsection{Discussion}

Distortional effects are very similar to those obtained in earlier experiments. Memory for target location is distorted away from the landmarks and away from the midpoint between the landmarks. Obviously, the virtual line connecting the landmarks has a special role as an axis of symmetry in the vector field. The purpendicular axis through the midpoint is also an obvious symmetry axis.

In the horizontal and vertical conditions, intrinsic axes of the stimuli were aligned with the allocentric horizontal and vertical, which might be expected to enhance the strength, regularity, and symmetry of distortional effects. However, comparison of straight and oblique stimulus orientations show no qualitative difference when intrinsic axes form a $45^{\circ}$ angle with the allocentric axes, except for smaller horizontal errors near the landmarks. Variable errors for the four different orientations are also comparable, indicating that positional certainty is not compromised even though intrinsic and extrinsic systems of reference are misaligned.

These results show that distortional fields can be closely tied to intrinsic stimulus axes, even if these axes are misaligned with important environmental reference directions. In this sense, distortional fields might indeed be invariant with respect to rotation of the stimulus configuration, as predicted by Nelson and Chaiklin's (1980) and Watson's (1977) theories. However, there are some caveats. First, Ex- 
periment 6 will show that horizontal and vertical configurations of landmarks do not necessarily lead to identical patterns of distortion when extrinsic spatial cues (e.g., from the monitor eges) are eliminated. Second, Experiments 7 to 9 performed with configurations of three landmarks clearly show effects of stimulus orientation and alignment of salient stimulus axes with allocentric axes. However, in contrast to those experiments, the present experiment used a rectangular frame aligned with the monitor that might have reduced positional uncertainty so that a system of reference anchored to the stimuli could be used more efficiently. Such reduction of uncertainty might be a necessary precondition for rotational invariance to hold.

\subsection{Experiment 4}

The psychophysical method used in Experiments 1 to 3 only implies that a distorted representation is present during reproduction of memorized targets. It does not suffice to determine whether distortion occurs as a dynamical process, gradually building up during the masking interval as required by models assuming distorted representations in topographic neuronal maps. The purpose of this experiment was to investigate the time course of distortion effects in the two-landmark task by using a visual discrimination paradigm. Instead of reproducing a target location, participants now had to tell whether a target had been displaced to the left or to the right during the masking interval. In addition to the 400-ms mask used in Experiment 3, a 100-ms mask was employed to see whether effects of distortion were already present at this early stage.

Nelson and Chaiklin's (1980) model makes to predictions about the timecourse of distortions. In their view, distortions arise from the psychophysical power function for remembered target-to-landmark distance, which is not expected to change during the retention interval. Huttenlocher et al.'s model (1991), in contrast, is potentially dynamic because distortional effects depend on the spatial uncertainty in memory distributions here. Every factor that increases spatial uncertainty should also increase the magnitude of distortions. In fact, the authors could show that employment of a distractor task increased both scatter and bias in reproduced locations. The passing of time might have similar effects if memory representations become increasingly noisy during the retention interval, resulting in a gradual increase in spatial 
memory distortions.

Unlike the method employed by Diedrichsen \& Werner (submitted), where participants had to judge whether or not a dot had been shifted between two presentations, the participants' task in this experiment was to report the direction of target displacement rather than to simply detect its presence. This allows one to sample psychometric functions that can be readily analyzed by statistical standard procedures, yielding separate estimates for strategic bias and displacement sensitivity of individual participants. Discrimination paradigms have additional advantages over detection paradigms in allowing for much better control of response bias arising, e.g., from observers with very liberal or very conservative response criteria (MacMillan \& Creelman, 1991).

The underlying logic of the experiment is as follows. Assume that a target is presented in a region of the display where the landmark induces a rightward bias in the memory representation of the target. Now consider that the target is physically displaced to the right during the masking interval. Because the memory representation has also drifted to the right, the displacement should appear small, and the likelihood of participants reporting a rightward displacement should also be small. Compare this with the situation in another region of the display where a leftward bias of the memory representation is induced. If the target is still physically displaced to the right, the apparent displacement should be large, and the likelihood of reporting a rightward displacement should be large, too, leading to a leftward shift of the psychometric function.

\subsubsection{Method}

Participants.

Six undergraduate students (age 22 to 29, all female, two of them left-handed) of the Institute of Psychology at the University of Göttingen participated for course credits or for a payment of 12,- DM per hour. Their vision was normal or corrected-tonormal.

Apparatus.

The setup was the same as in Experiment 3, only that the viewing distance was $80 \mathrm{~cm}$. 
a) Possible landmark and target locations.

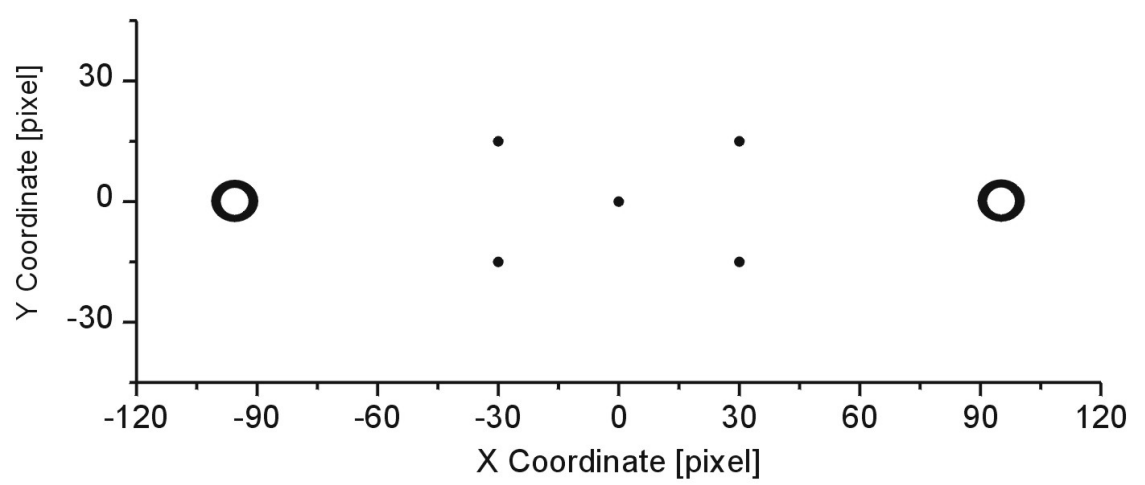

b) Time course of a trial.

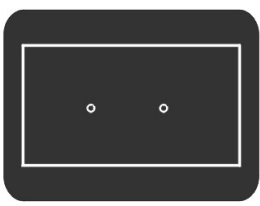

Landmarks: $500 \mathrm{~ms}$

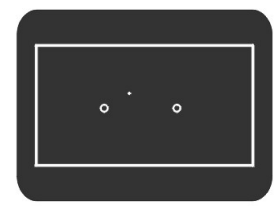

Target: $500 \mathrm{~ms}$

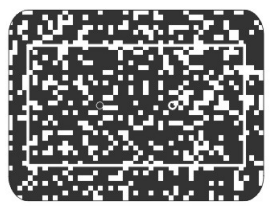

Dynamical mask: 100 or $400 \mathrm{~ms}$

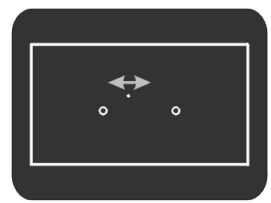

Target displaced to the left or right by $0-3 p x$

Fig. 14a: Landmarks and possible target locations in Experiment 4. b: Time course of a trial.

\section{Stimuli.}

To allow for direct comparisons between experiments, stimuli were the same as in Experiment 3, with the following exceptions (Fig. 14a). Only one stimulus orientation was used, so that the two landmarks were always horizontally aligned. The landmarks were filled rather than open circles in this experiment. There were only five target positions: one at the center of the screen, the other four positions at $y$ coordinates of $\pm 15 \mathrm{px}\left( \pm 0.54^{\circ}\right)$ and $x$ coordinates of $\pm 30 \mathrm{px}\left( \pm 1.07^{\circ}\right)$. These targets corresponded to the five innermost target positions from Experiment 3 . They were chosen because they had shown strong distortions along the horizontal coordinate in Experiment 3 , and this distortion had been independent of display orientation. Furthermore, they were all near the center of the display, which should reduce possible effects of stimulus eccentricity when fixating on the center. Dynamic masks were used as described in Experiment 1, one with a duration of $100 \mathrm{~ms}$ and one with a duration of $400 \mathrm{~ms}$. 


\section{Procedure.}

A trial began with the presentation of the two landmarks within the white rectangular frame as in Experiment 3 (Fig. 14b). After $500 \mathrm{~ms}$, the target appeared at one of the five possible locations and remained on the screen for $500 \mathrm{~ms}$ before it was replaced by a 320 x 200 px dynamic mask for either 100 or 400 ms. Immediately following mask presentation, the target was presented again, but this time with a displacement of 0 to $3 \mathrm{px}$ to the left or right of its original position. Participants were not informed that in some cases no displacement occured. The landmarks remained visible until the participant responded and were also visible during the masking interval. The participants' task was to indicate whether the target had been displaced to the left or right by pressing the appropriate key ("4" for "left", "6" for "right" on the numerical pad of the computer keyboard) with the index or ring finger of their right hand, respectively. The instruction emphasized accuracy rather than speed and encouraged participants to guess when they were not sure about the direction of target displacement. Keypress responses elicited a $2000 \mathrm{~Hz}, 100 \mathrm{~ms}$ tone for feedback that the response had been registered, and a warning tone $(100 \mathrm{~Hz}, 500 \mathrm{~ms})$ when a key other than the two permitted was used. After an intertrial interval of $500 \mathrm{~ms}$, the next trial began. Throughout the experiment, no feedback concerning the level of performance was given.

Stimulus conditions were counterbalanced such that each combination of target position, mask duration, and target displacement occured randomly and equiprobably, with each combination appearing once every two blocks. The center target position appeared twice as often as any other target position to yield equal numbers of observations for horizontal and vertical coordinates.

Each participant took part in four sessions of 16 blocks with 42 trials each. Each session started with an additional practice block of 42 trials. At the beginning of the first session, participants received some training in the discrimination task in a short demonstration program using larger target displacements. After the last session, they were debriefed and received an explanation of the purpose of the experiment.

\section{Data treatment.}

For the following analyses, response times shorter than $100 \mathrm{~ms}$ and longer than 999 ms were excluded (0.92 \%). Additionally, all trials were excluded where a 
participants' response times were more than three standard deviations above or below her average response time (2.37\%). Practice blocks were not analysed.

\subsubsection{Results}

Results are shown in Figure 15. Psychometric functions were analysed by multiple logistic regression (Agresti, 1996). Logistic regression fits models of the type $f\left(x_{1}, x_{2}\right)=\frac{\exp \left(a+b x_{1}+c x_{2}+d x_{1} x_{2}\right)}{1+\exp \left(a+b x_{1}+c x_{2}+d x_{1} x_{2}\right)}$ to a binomial dependent variable, where $x_{1}$ and $x_{2}$ are two independent variables and the $x_{1} \cdot x_{2}$ term describes the interaction of the variables. The logic extends to higher-order factorial designs. All such models yield sigmoid functions with values between 0 and 1 , with the regression weights describing whether the slope increases or decreases with a particular variable. The SPSS ${ }^{\odot}$ algorithm used here works with a Wald statistic, which is constructed by dividing the estimated regression weight by its asymptotic standard error, the resulting variable having an approximate $\chi^{2}$ distribution. The statistic is reported here as $W(\mathrm{df})$, with appropriate degrees of freedom. Because the goal here is to assess the magnitude of theoretically important effects rather than to find the "best" regression model, a saturated model was chosen a priori containing the effects of target displacement, mask duration, and the expected direction of spatial distortion, as well as all interactions among these variables. The intercept term was set to zero.

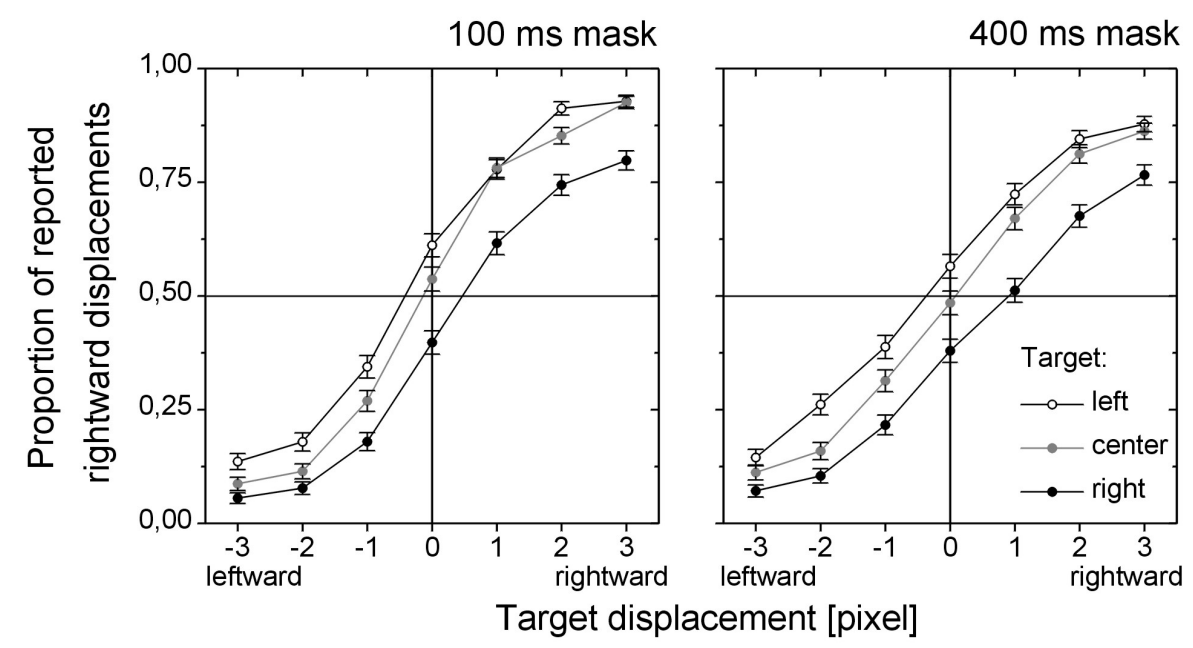

Fig. 15: Psychometric functions from Experiment 4, separately for both mask durations. Standard errors are between trials. 
As expected, there was a main effect of target displacement, indicating that the experiment yielded psychometric functions with a positive slope, $b=1.016, W(1)$ $=648.450, p<.001$. Logistic functions gave an excellent fit to the observed cell means, $R^{2}=.990$. There was also a main effect of mask duration, $b=-0.069, W(1)=$ $30.715, p<.001$, and an interaction of mask duration with target displacement, $b=$ $0.172, W(1)=50.741, p<.001$, reflecting the fact that psychometric function were flatter at the longer masking interval, which indicates that participants could discriminate the direction of displacement less precisely.

Most important, there was a clear main effect of expected direction of distortion, indicating that participants had a bias remembering the displacement in the direction predicted from the distortion found in earlier experiments, $b=-0.017, W(1)=$ 40.853, $p<.001$ : leftward distortions led to leftward shifts of psychometric functions, while rightward distortions led to rightward shifts. None of the other interactions approached significance, all $p>$.140. Subsequent tests at each masking duration confirmed that psychometric functions were shifted in both directions compared to the neutral cases where the target had been presented at display center, all $b>.300 \mathrm{px}$, all $W(1) \geq 16.899$, all $p<.001$.

\subsubsection{Discussion}

Psychometric functions clearly indicate that some distortion takes place in visual memory between one presentation of the target and the next. These effects are in agreement with the results from Experiment 3, where the same targets were used for a reproduction task. For targets at the midpoint of the display, memory is veridical, and there is no discernible bias. For targets to the left or right of the center, however, psychometric functions are shifted in a way that suggests memory distortions away from the display center, as was observed in Experiment 3 as well as in earlier work (Diedrichsen, 1998; Diedrichsen \& Werner, submitted). Psychometric functions shift because a target translation is easier to detect when going against the direction of distortion than when going with it. As predicted, this leads to a tendency of reporting translations to the right for targets on the left side of the center and to report translations to the left for targets on the right side of the center.

The fact that psychometric functions are slightly flatter after a longer masking 
interval indicates increasing uncertainty about the direction of the target translation. This effect can be reconciled with Huttenlocher et al.'s model if it is assumed that memory distributions become increasingly fuzzy during the retention interval, which would result in larger truncation and prototype effects; it is not easily explained by Nelson and Chaiklin's (1980) model unless the psychophysical function for remembered target-to-landmark distance is allowed to change over time.

However, the increase in uncertainty is not accompanied by an increase in memory distortions. Instead, the amount of distortion is the same after a 100-ms mask than after a 400-ms mask; the effect seems to have reached an asymptote as early as $100 \mathrm{~ms}$ post-stimulus. Recent data from our laboratory using the same paradigm but different masking intervals show that most of the distortion occurs within $100 \mathrm{~ms}$, but there is little further increase in distortion between $200 \mathrm{~ms}$ and $400 \mathrm{~ms}$. This is at odds with the results of Diedrichsen and Werner (submitted) suggesting that memory distortions develop over a time of roughly $400 \mathrm{~ms}$, with much less distortion after $100 \mathrm{~ms}$. One reason for this might be possible response biases in Diedrichsen and Werner's procedure. In their task, participants did not have to indicate the direction in which a target had been translated but only reported on whether the target had been moved or not. With such a task, it is difficult to disentangle tendencies to prefer "yes" or "no" responses from the effects of genuine memory distortions. Participants might have different response biases at different masking intervals; e.g., if participants were more lenient towards reporting a target translation at longer masking intervals than at shorter intervals, this would lead to underestimation of memory distortions at short masking intervals. In contrast, having people report the apparent direction of target translation is a discrimination rather than a detection task and is much less affected by such response tendencies (MacMillan \& Creelman, 1991). Of course, one can only speculate about the magnitude and direction of response tendencies in Diedrichsen and Werner's task.

Together, Experiments 3 and 4 show that spatial distortions develop in a regular, symmetric fashion in two-landmark displays. While the distortion away from the midpoint is in line with Nelson and Chaiklin's (1980) model, the distortion away from the landmarks runs counter to the model. Distortions are shown to arise dynamically during the masking interval, which is not easily predicted by Nelson and Chaiklin's model but is a straightforward prediction of models assuming that spatial memory distortions develop in topographical neuronal maps. The pattern and dynamics of 
spatial biases alone is also consistent with Huttenlocher et al.'s (1991) model if it is assumed that observers place category boundaries at the landmarks, at the midpoint, and possibly at the connecting line between the landmarks, which would suffice to predict distortions generated by truncation and prototype effects. However, it is not yet clear whether the Huttenlocher et al. model could also predict the pattern of variances. Discussion of variance effects is deferred to Experiments 5 and 6. 


\section{Part Ilb: Two Landmarks - Nonlinear Organisation of the Visual Field}

Predicting distortional fields of multiple landmarks.

Can the distortional fields of two landmarks be predicted from the distortional fields of the single landmarks presented individually? This is a fundamental problem to be solved if one wants to make predictions for arbitrary configurations of landmarks. Obviously, without a theory of how landmarks combine we would be forced to develop a new model for every new landmark configuration we encounter.

Although some of the models of spatial memory distortions proposed in the past make some suggestions as to how landmarks may combine, neither of them really addresses the issue in a systematic way. Nelson and Chaiklin's (1980) model is the only one that explicitly assumes a weighting function for combining landmarks which relates effects of individual landmarks to the combined distortional field. While this weighting function is assumed to decrease monotonously with landmark distance, the amount of distortion is assumed to increase with distance from the landmark, which would counteract the diminishing effects of the weighting functions. In any case, their model assumes that distortional effects of each landmark have to spread throughout the entire field, at least as far as the radius of the circle used in their displays, to account for the effects.

In contrast, models based on local interactions within topographical memory maps (e.g., Desimone \& Duncan, 1995, Suzuki \& Cavanagh, 1997) predict that distortional effects are locally restricted to the vicinity of the focus of attention. Therefore, the distortional pattern around a landmark should remain unchanged if a second landmark is presented at sufficient distance, provided that the first landmark still has the focus of attention. In a two-landmark situation, the landmark closest to the target would be expected to dominate the size and direction of the distortion. This would lead to a self-organizing process similar to Voronoi partitioning of the visual field into discrete regions.

Finally, although the Huttenlocher et al. (1991) model lacks any explicit statement about how landmarks combine, it allows for qualitative differences to arise when advancing from a one-landmark to a two-landmark configuration. With two landmarks, new spatial categories emerge, and the number of category boundaries and 
category prototypes increases correspondingly, possibly leading to a different organization of the field.

\section{Linear and partition models.}

Instead of proposing a detailed model with very specific assumptions about how individual landmark fields combine, I will focus on the most important formal properties such a model should have. One very important distinction between models is whether they behave linearly or nonlinearly. More specifically, we will contrast several models of special theoretical interest: linear models assuming that the two-landmark field can be found by linear combination of single-landmark fields, and a partition model assuming that distortional fields are dominated by the nearest landmark.

One very important class of linear systems is the one satisfying the superposition principle. This principle states that the system's response to a sum of stimuli is simply the sum of its responses to the individual stimuli, $f(a+b)=f(a)+f(b)$. By the same token, scaling the stimulus in magnitude will also scale the response by the same factor, $f(\tau \cdot a)=\tau \cdot f(a)$. Superposition systems are abundant in natural science because superposition holds for all known physical force fields, e.g., gravity and electromagnetism. The theoretical importance of such systems lies in their generalizability beyond extremely simple input conditions: If one knows how the system responds to a single unit of input, one can infer how it will respond to any arbitrarily complex combination of such units. Superposition models have been very successfully used in vision science as a model of early brightness and color perception (Wandell, 1995). Applied to our problem, the superposition principle states that in order to obtain the distortional field of two landmarks, we only have to vectorially sum the distortional fields of the individual landmarks measured alone. Obviously, this superposition model is a special case of a more general two-parameter linear model allowing each field to be multiplied by some constant before summation, with superposition resulting when both weights are 1.

An entirely different way of combining individual fields is described by partition models: the influence of the left landmark might be larger in the left half of the field while the right landmark might dominate the right half. In this case, the two landmarks would segment the visual field, locally restricting each other's region of influence. In this thesis, the partition model is implemented by multiplying each single-landmark field by a simple step function that is 1 on the side of the landmark, 0 on the opposite 
side, and undefined on the vertical midline (the partition boundary) between the landmarks. These weighted fields are then superimposed, similar to cutting the single-landmark fields in the middle and glueing the landmark ends together again. Effects on the midline can be predicted without further mathematical assumptions as the limiting values found by averaging their nearest neighbours to the left and right of the midline. 1

While superposition is a typical property of simple physical processes spreading independently through a passive medium, partitioning is more indicative of processes competing for space or spatially distributed resources. In a partition model, influences of the landmarks are not independent but are subject to mutual spatial restriction. Attentional models like that of Suzuki and Cavanagh (1997) would be expected to behave like a partition model because the distortional effects are spatially restricted to the vicinity of the landmarks. In contrast, Nelson and Chaiklin's (1980) model might resemble either a partition or a linear model depending on how sharply the weighting functions decrease with distance from the landmark compared to the function describing the amount of distortion. Note that in both the linear and partition models, predictions follow strictly from stimulus geometry, and generalization to more complex configurations of landmarks is straightforward. From a model-fitting point of view, the superposition and partition models are among the most simple models conceivable because they do not have any free parameters.

Experiment 5 investigates whether the distortional fields of single landmarks combine linearly or by partition. As in previous experiments, the participants' task was to reproduce the location of a briefly presented target. In order to measure individual and combined fields, either the left, the right, or both landmarks were presented in random order. Experiment 6 replicates these findings with a refined laboratory setup designed to minimize extraneous spatial references. It also extends the results to vertical landmark configurations to check whether distortional fields are invariant regarding the orientation of the landmarks.

\footnotetext{
${ }^{1}$ Several other models are possible. For example, one could use weights of 0.5 for points on the midline, but this would imply a linear combination rule.
} 


\subsection{Experiment 5}

\subsubsection{Method}

Participants.

Six students (age 21 to 23, all female, all right-handed) of the Institute of Psychology at the University of Göttingen participated for course credits or for a payment of 12,- DM per hour. Their vision was normal or corrected-to-normal.

a) Possible landmark and target locations.

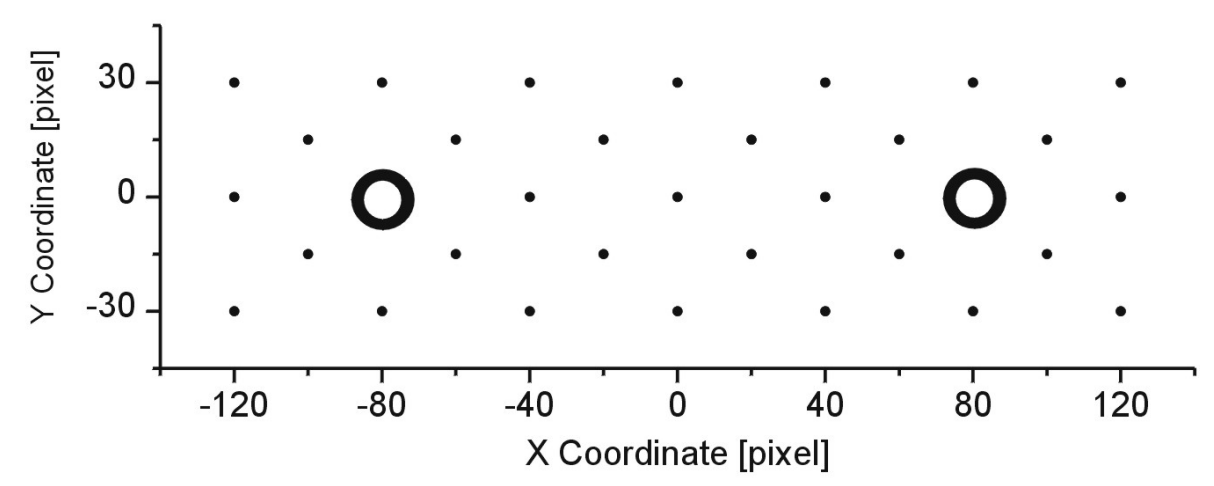

b) Time course of a trial.

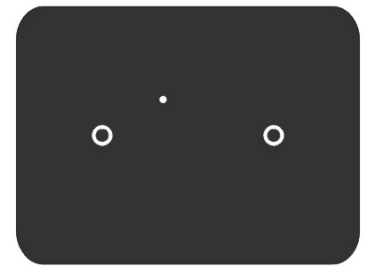

Target with one or two landmarks: $1500 \mathrm{~ms}$

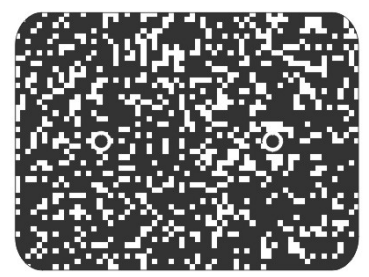

Dynamical mask (landmarks still visible): $500 \mathrm{~ms}$

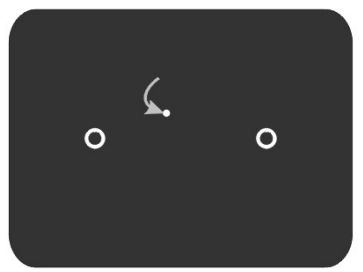

Reproduction of target location with mouse cursor

Fig. 16a: Possible landmark and target locations in Experiment 5. b: Time course of a trial.

\section{Stimuli.}

The electronic equipment was the same as in Experiment 1. All stimuli were presented against a black background $\left(0.01 \mathrm{~cd} / \mathrm{m}^{2}\right)$. Landmarks were green unfilled circles $\left(28.7 \mathrm{~cd} / \mathrm{m}^{2}\right), 11 \mathrm{px}\left(0.31^{\circ}\right)$ in diameter and $80 \mathrm{px}\left(2.27^{\circ}\right)$ to the left or right of the center of the screen. The target was a small white dot $\left(43.0 \mathrm{~cd} / \mathrm{m}^{2}\right)$ with a diameter of 3 px, presented at one of the 33 possible locations shown in Fig. 16a, with the 
restriction that no target was presented at a position currently occupied by a landmark. The whole grid spanned a visual area of about $6.80^{\circ} \times 1.70^{\circ}$. A dynamical mask was used as described in Experiment 1.

\section{Procedure.}

A trial began with the appearance of the target together with either the left, right, or both landmarks (Fig. 16b). Participants were allowed to inspect this stimulus for 1500 ms under free viewing conditions, without any restriction of eye movements. The target was then replaced by the dynamic mask for 500 ms while the landmarks remained visible. Immediately after the masking interval, a mouse cursor looking exactly like the target appeared. In order to avoid the inititial cursor position to act as a confounding spatial reference, the cursor always appeared in the center of a landmark (if both landmarks were present, it appeared randomly in one or the other). The participants' task was to use the mouse cursor to reproduce the target's location as exactly as possible and to press the left mouse button when finished. The button press elicited a $1000 \mathrm{~Hz}, 100 \mathrm{~ms}$ tone for feedback that the response had been registered. After an intertrial interval of 500 ms, a new trial began. Participants were instructed to work quickly, but it was emphasized that accuracy rather than speed was important in this task.

Stimulus conditions were counterbalanced such that each combination of landmark configuration (left, right, both), starting position of the mouse cursor (left, right), and target position occured quasi-randomly and equiprobably, with each combination appearing once every five blocks. After each block, participants received summary feedback about their average euclidean deviation from the true target position, rounded to the nearest pixel.

Participants performed three sessions of 15 blocks with 38 trials each. Each session started with an additional practice block of 38 trials with stimuli drawn randomly from the experimental blocks. Practice trials were not analyzed. After the final session, participants were debriefed and received an explanation of the purpose of the experiment.

\section{Data treatment.}

Trials with reponse times faster than 100 ms or slower than 5000 ms were excluded because these trials likely reflected anticipatory or guessing behavior. Trials 
with a target deviation larger than $30 \mathrm{px}$ in either the horizontal or vertical component were also excluded. From the remaining data, trials with horizontal or vertical error more than three standard deviations above or below the average deviation of a given participant were discarded. The whole procedure eliminated $5.76 \%$ of the raw data. Because participants differed considerably in the absolute magnitude of the distortional effects they produced, the z-standardized response distributions computed separately for each participant entered the analysis.

\subsubsection{Results}
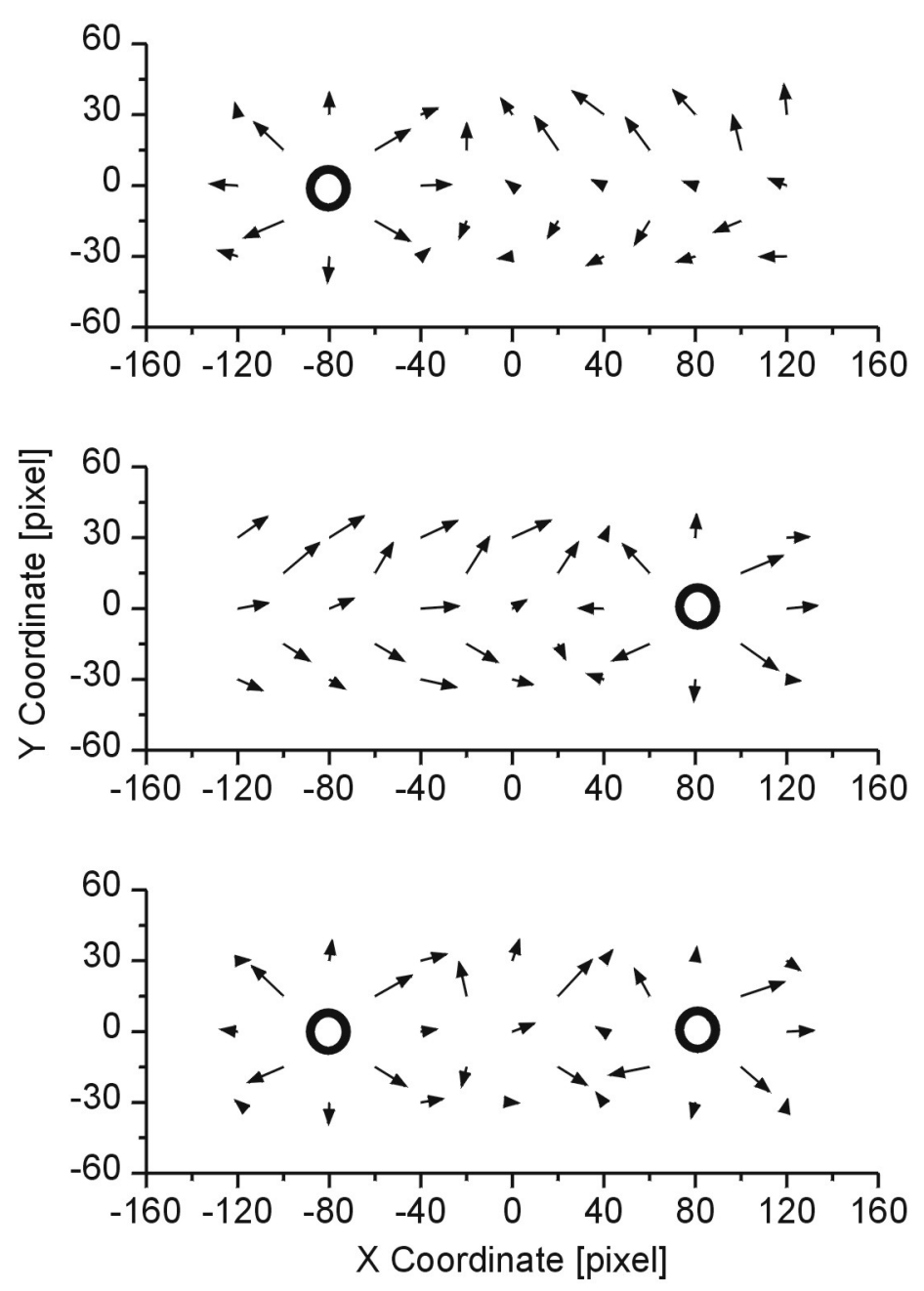

Fig. 17: Distortional fields in Experiment 5. Arrows are magnified by a factor of 3 for readability. 


\section{Basic patterns.}

Fig. 17 shows the distortional fields in the different landmark conditions. Reproduced target positions were clearly distorted away from landmark stimuli. Distortion occured in a characteristic pattern depending on where the landmarks were located and whether there were one or two of them, displaying obvious regularity and symmetry.

In all conditions, the largest distortions occured in the vicinity of the landmarks, where reproductions were biased away from the landmark in a radial fashion. In the display half where no landmark was presented, distortion was towards the landmark and away from the connecting line. Importantly, with both landmarks present, there was some additional distortion away from the midpoint between the landmarks.

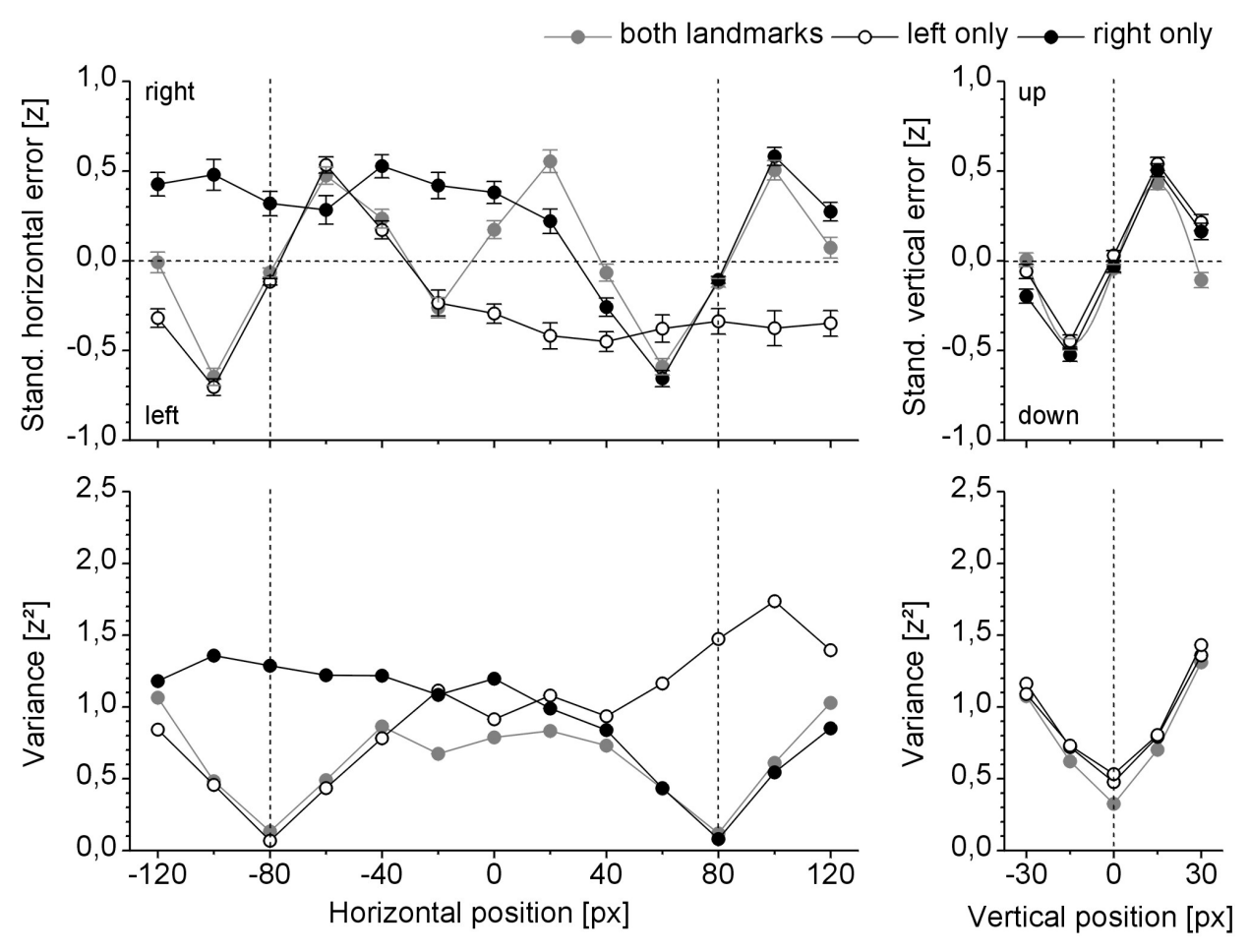

Fig. 18: Average systematic and variable error (upper vs. lower panel) in the horizontal and vertical direction (right vs. left panel). Standard errors are between trials.

It is striking that the distortional fields in the vicinity of single landmarks are very similar to the corresponding regions in the both-landmark condition. Fig. 18 shows the patterns of constant and variable error separately for the horizontal and vertical components of distortion. It can be seen that the local pattern of biases and 
variances around a landmark is the same irrespective of whether the other landmark is present. These results give important boundary conditions for modelling, strongly suggesting some kind of partitioning model.

MANOVA confirmed that the vectors of memory distortions depended on target position, $\Lambda(60,298)=.393, p<.001$. There was a main effect of landmark condition, $\Lambda(4,18)=.217, p<.01$, mainly in the horizontal coordinate; horizontal: $F(2,10)$ $=6.174, p<.05$; vertical: $F(2,10)=2.724, p>.10$. This effect was mainly due to the fact that the average standardized horizontal distortion was to the left in the left landmark condition, to the right in the right landmark condition, and neutral in the twolandmark condition. Importantly, there was an interaction of target position and landmark condition, $\Lambda(120,598)=.395, p<.001$, confirming that the patterns of distortion differed across landmark conditions.

Models of systematic error.

Each model's fit to the data was evaluated by linear regression of the values observed in the two-landmark condition against the values predicted by each model, separately for each participant. With perfect model fit, the slope and intercept parameters should be 1 and 0 , respectively. Horizontal and vertical errors entered the process simultaneously. The two-parameter linear model was fitted to data by leastsquares multiple regression.

Comparisons of observed and predicted values of distortion are shown in Fig. 19. The best-fitting linear model adds the left and right-landmark fields with weights of 0.344 and 0.312 , respectively, thereby approximating some kind of averaging of distortional fields. It grossly underestimates the amount of horizontal bias, and it fails to predict the distortion away from the midpoint.

Although it is not the best-fitting model in terms of least squares, the superposition model makes slightly more reasonable estimates than the two-parameter linear model. However, there are still substantial prediction errors, including a general overestimation of distortions for most horizontal positions, a predicted distortion towards the midpoint rather than away from it, and distortions directly above and below the landmarks which are not present in the data. 


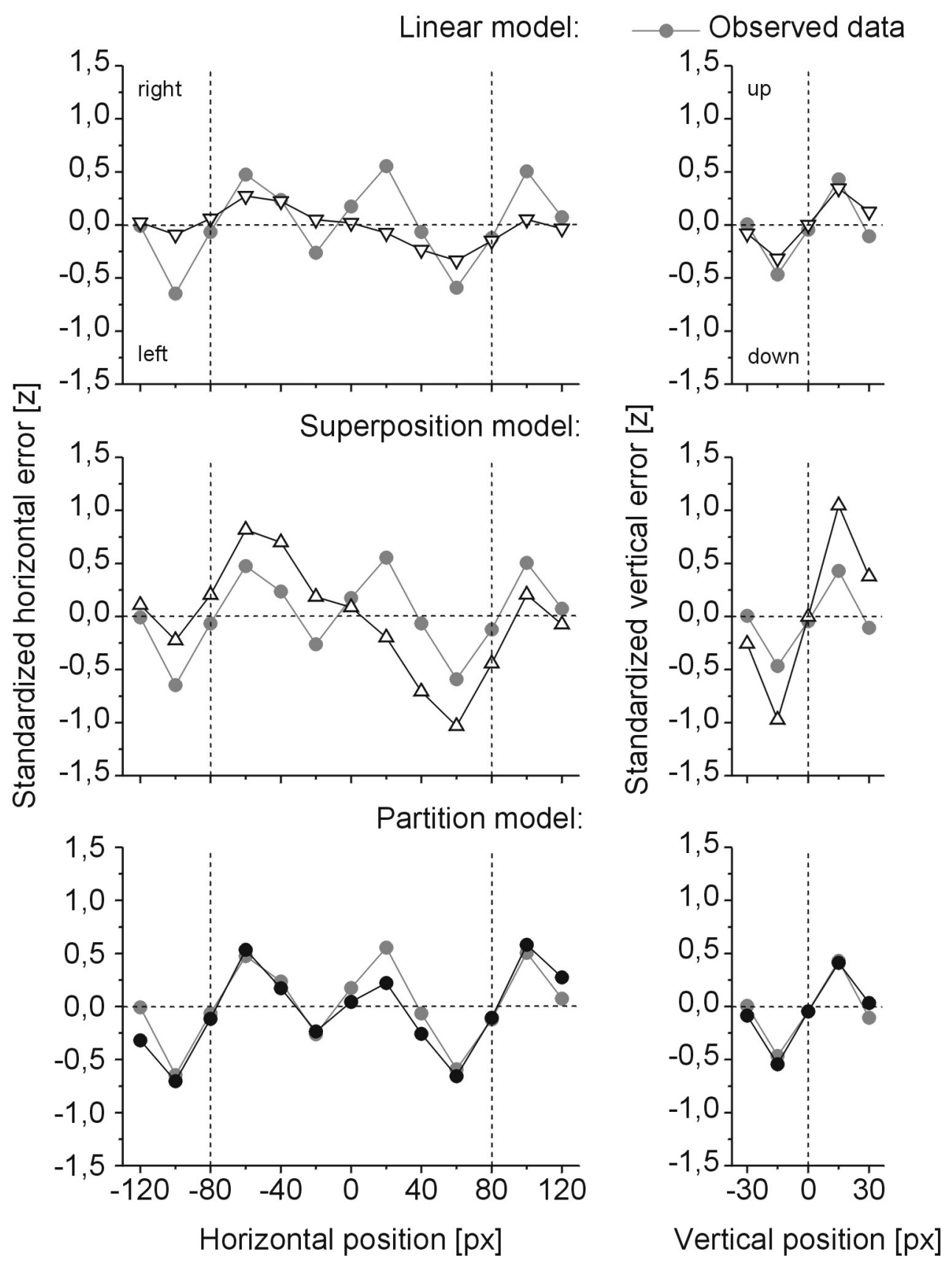

Fig. 19: Data and predictions of systematic error for the three models. Horizontal error is in the left, vertical error in the right panel.

Predictions for the partition model are straightforward except for the points on the vertical midline where the weighting function is undefined. These predictions were derived by finding a limiting value, averaging the rightmost points in the left-landmark field and the leftmost points in the right-landmark field to arrive at a "good guess" for the points in between without further mathematical assumptions. In contrast to both linear models, the partition model fits the data closely except for a slight underesti- 
mation of distortion away from the midpoint. It also fits the pattern of vertical distortions.

Table 1: Goodness-of-Fit of the models in Experiment 5

\begin{tabular}{|c|c|c|c|c|}
\hline Effect: & slope & & intercept & $\underline{\mathrm{R}^{2}}$ \\
\hline \multicolumn{5}{|c|}{ General Linear Model } \\
\hline Constant Error: $\mathrm{x}$ & 1.331 & & 0.043 & .342 \\
\hline Constant Error: y & 1.183 & & -0.052 & .823 \\
\hline \multicolumn{5}{|l|}{ Superposition Model } \\
\hline Constant Error: $\mathrm{x}$ & 0.432 & * & 0.032 & .339 \\
\hline Constant Error: $\mathrm{y}$ & 0.387 & * & -0.051 & .822 \\
\hline \multicolumn{5}{|l|}{ Partition Model } \\
\hline 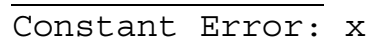 & 0.877 & & 0.061 & .854 \\
\hline Constant Error: y & 0.902 & & 0.006 & .963 \\
\hline Variable Error: $\mathbf{x}$ & 0.737 & & 0.153 & .723 \\
\hline Variable Error: y & 0.875 & & 0.034 & .985 \\
\hline
\end{tabular}

Table 1 shows that the partition model fits the data more closely than the other models, with all slope parameters near 1 , all intercept parameters near 0 , and high $R^{2}$ values. In contrast, the slope parameters of the two-parameter linear model are slightly too large, indicating underestimation of effects, while those of the superposition model are significantly too small, indicating overestimation of effects. For the pattern of horizontal biases, both models have very unsatisfactory $R^{2}$ values.

\section{Models of variable error.}

Despite the simplicity of the linear models, predictions of variances from them are not straightforward unless it could be assumed that the left and right landmark fields independently create their own pattern of variances, i.e., that landmarks were the only sources of variance in the visual field. If this were the case, variance fields would superimpose, summing with the squares of their weights. However, this assumption is quite unreasonable because it predicts that there would be no variance in reproductions if no landmarks were presented. This is at odds with the finding from Experiment 2 that response variance near the landmarks is reduced compared to a control condition where no landmarks are presented. 


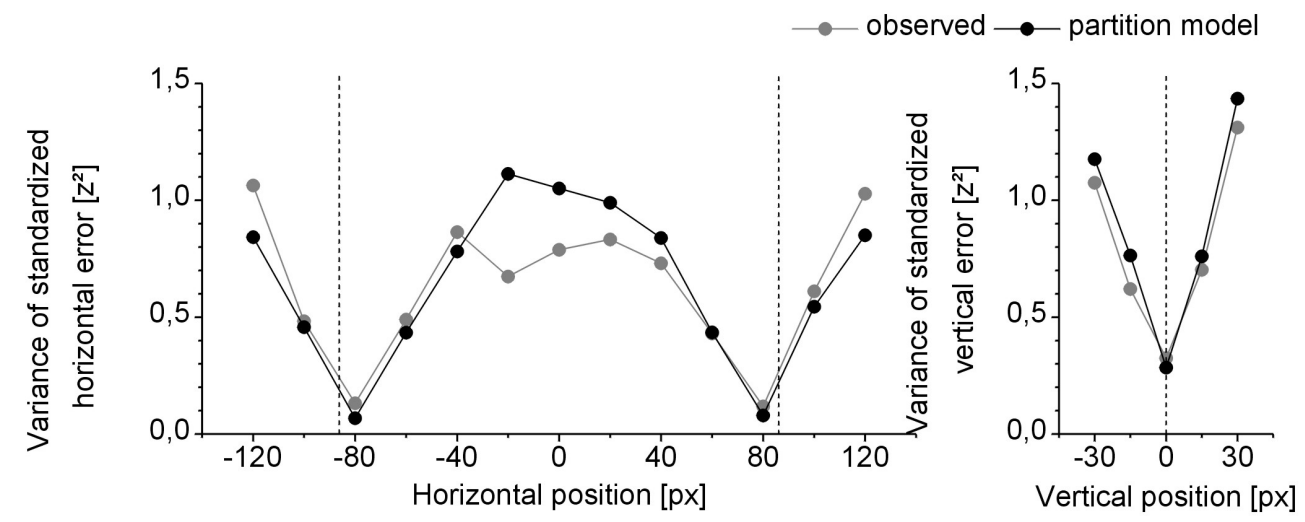

Fig. 20: Data and predictions of variable error for the partition model. Horizontal error is in the left, vertical error in the right panel.

In contrast, variance predictions for the partition model follow readily from the formulation of the model: variances in the left half of the two-landmark field are estimated by variances in the corresponding part of the left-landmark field, and the right half is treated analogously. Again, variance predictions for points located on the vertical midline were derived by taking a limiting value, averaging the left and right neighboring points. Fig. 20 shows that the partition model makes reasonable predictions about the empirical pattern of variances for both horizontal and vertical coordinates, as is also reflected in the regression parameters in Table 1. However, the model overestimates the amount of variable error at the vertical midline.

\subsubsection{Discussion}

Consistent with Experiments 3 and 4, spatial visual memory for briefly presented targets is distorted away from landmark stimuli, following a complex but regular and symmetrical pattern. The major features of this pattern are locally restricted radial distortions away from the landmarks, distortions away from the connecting line and towards the landmarks in the rest of the display, and additional distortion away from the midpoint between two landmarks. The pattern of spatial biases around the connecting line might indicate a special role for the allocentric horizontal or an influence of the imaginary line connecting the landmarks when both landmarks are presented. Alternatively, it might be an artifact of the visible monitor frame or some other spatial cues visible during the experiment. 
The two-parameter linear model predicts that the single-landmark distortional fields add after appropriate weighting with some constants. However, the weights giving the best fit to the data in terms of least squares lead to dramatic underestimation of systematic error. As a special case of this model when both weights are set to 1 , the superposition model assumes that the distortional field in the two-landmark condition can be predicted by simply adding the single-landmark fields, a procedure appropriate for physical force fields. When this is done, this model also deviates from the data in several important respects. First, it overestimates the amount of distortion in most regions of the display. Second, it predicts that positions directly above or below the landmark are distorted towards the center although such distortions are not found empirically. Third, it predicts distortion towards the center of the display rather than away from it. These deviations are severe both quantitatively and qualitatively, and on these grounds both linear models must be rejected.

In contrast, the predictions of the partition model are quite accurate. It closely fits the pattern of distortion both in the horizontal and vertical components, correctly predicting points of zero distortion. It even predicts some distortion away from the midpoint between the landmarks, although the magnitude of this effect is somewhat underestimated. Importantly, it can be successfully used to estimate the pattern of variable error: predicted variances have the correct order of magnitude and closely follow the observed pattern near the landmarks. Systematic deviations are present, however, at the center of the display where variances are overestimated. These deviations suggest that a partition principle may hold in most areas of the display, but an additional process might be needed to explain distortions near the display center. Further discussion is deferred to the end this section.

\subsection{Experiment 6}

In Experiment 5, landmarks and targets were presented on an ordinary computer monitor, with the monitor edges clearly visible. Thus, participants might have used spatial reference stimuli other than the landmarks to encode the location of the target dot. The laboratory setup was therefore modified to reduce these possible artefacts as effectively as possible. Participants were seated at the wider end of a large funnel, with the monitor situated at the narrow end such that the laboratory environment was blocked from view and the monitor edge was circular rather than rectan- 
gular. Because the experiment was performed in darkness and the visual stimulation was chosen to keep the participants light-adapted throughout the session, the circular monitor edges were clearly visible only during the masking interval and almost invisible during the presentation of landmarks and target.

Apart from improving the laboratory conditions, vertical in addition to horizontal configurations of landmarks were used to check whether distortional fields are invariant regarding the orientation of the display or differ when stimuli are aligned with the allocentric vertical instead of the horizontal. Most important, this was also a critical test to see whether the conclusions drawn from Experiment 5 about the superposition and partition models generalized to a different landmark configuration.

\subsubsection{Method}

\section{Participants.}

Eight students (age 21 to 26,7 of them female, all of them right-handed) participated for course credits or for a payment of 15,- DM per hour. Their vision was normal or corrected-to-normal.

Apparatus.

The electronic equipment was the same as in Experiment 1. The funnel was made of a homogenously textured fabric and left only a circular central portion (17 $\mathrm{cm}, 8.84^{\circ}$ ) of the screen where stimuli were presented at a viewing distance of 110 $\mathrm{cm}$. The laboratory environment was completely blocked from view. The experiment was performed in darkness, with the lighting switch within reaching distance of the participant.

\section{Stimuli.}

As before, all stimuli were presented against a black background $\left(0.01 \mathrm{~cd} / \mathrm{m}^{2}\right)$. Landmarks were green unfilled circles $\left(2.95 \mathrm{~cd} / \mathrm{m}^{2}\right), 9 \mathrm{px}\left(0.26^{\circ}\right)$ in diameter and 64 px $\left(1.82^{\circ}\right)$ to the left or right of the center of the screen. The target was a small gray $\operatorname{dot}\left(10.8 \mathrm{~cd} / \mathrm{m}^{2}\right)$ with a diameter of $3 \mathrm{px}\left(0.09^{\circ}\right)$. The grid of possible targets was similar to that used in Experiment 1, but the overall size of the configuration was scaled to approximately $73 \%$ to accomodate the restricted display size, spanning a visual angle of about $6.36^{\circ} \times 1.36^{\circ}$. In addition to the horizontal configuration of landmarks and targets, there was a vertical configuration where the landmarks and the grid of 
possible target locations were rotated by $90^{\circ}$. Instead of the dynamical noise mask, a simple brightness mask was used where the whole display was set to maximum intensity $\left(43.0 \mathrm{~cd} / \mathrm{m}^{2}\right)$. Brightness rather than pattern masking was used to keep the participants light-adapted so that the circular monitor edges were barely visible during presentation and reproduction (remember that Experiment 2 had established that the brightness mask was as effective as the dynamic mask). The brightness mask also excluded the potential strategy of using the black and white mask elements as local spatial cues.

\section{Procedure.}

The time-course of events within a trial was identical to that of Experiment 5. The only exception was that the mouse cursor no longer started from one of the two landmark positions but randomly from one of eight positions orderly arranged around the center of the display, at a radius of $120 \mathrm{px}\left(3.10^{\circ}\right)$. The cursor was not visible unless moved by at least $15 \mathrm{px}\left(0.39^{\circ}\right)$. This manipulation assured that the initial cursor position was unpredictable, which had not been the case in Experiment 5 in those conditions where only a single landmark had been presented.

Apart from these changes in stimulus conditions, the procedure was the same as in Experiment 5. Participants performed four sessions of 15 blocks with 38 trials each. Each session started with an additional practice block of 38 trials. After the final session, participants were debriefed and received an explanation of the purpose of the experiment.

\subsubsection{Results}

Data treatment proceeded as described in Experiment 5. The trimming procedure eliminated $3.05 \%$ of the raw data.

\section{Basic patterns.}

Fig. 21 and 22 show the distortional fields in the horizontal and vertical conditions, the latter normalized to horizontal orientation by a $90^{\circ}$ rotation counterclockwise. In both conditions, reproduced target locations in the vicinity of landmarks were biased away from the landmarks. When both landmarks were present, there was some additional distortion away from the midpoint between the landmarks. Elimination of monitor edges as a spatial reference frame seems to have led to additional 
biases that span the entire distortional field. In the horizontal condition, a pronounced downward bias can be seen in the lower half of the distortional field. In the vertical condition, this bias is even more pronounced, especially when only the lower landmark is presented. While in Experiment 5 distortions arranged symmetrically around the connecting line between the landmarks, this line seems to have little influence on the vector fields in the single-landmark horizontal conditions. In contrast, the orthogonal (vertical) midline in vertical configurations is a line of symmetry comparable to the horizontal midline in Experiment 5.
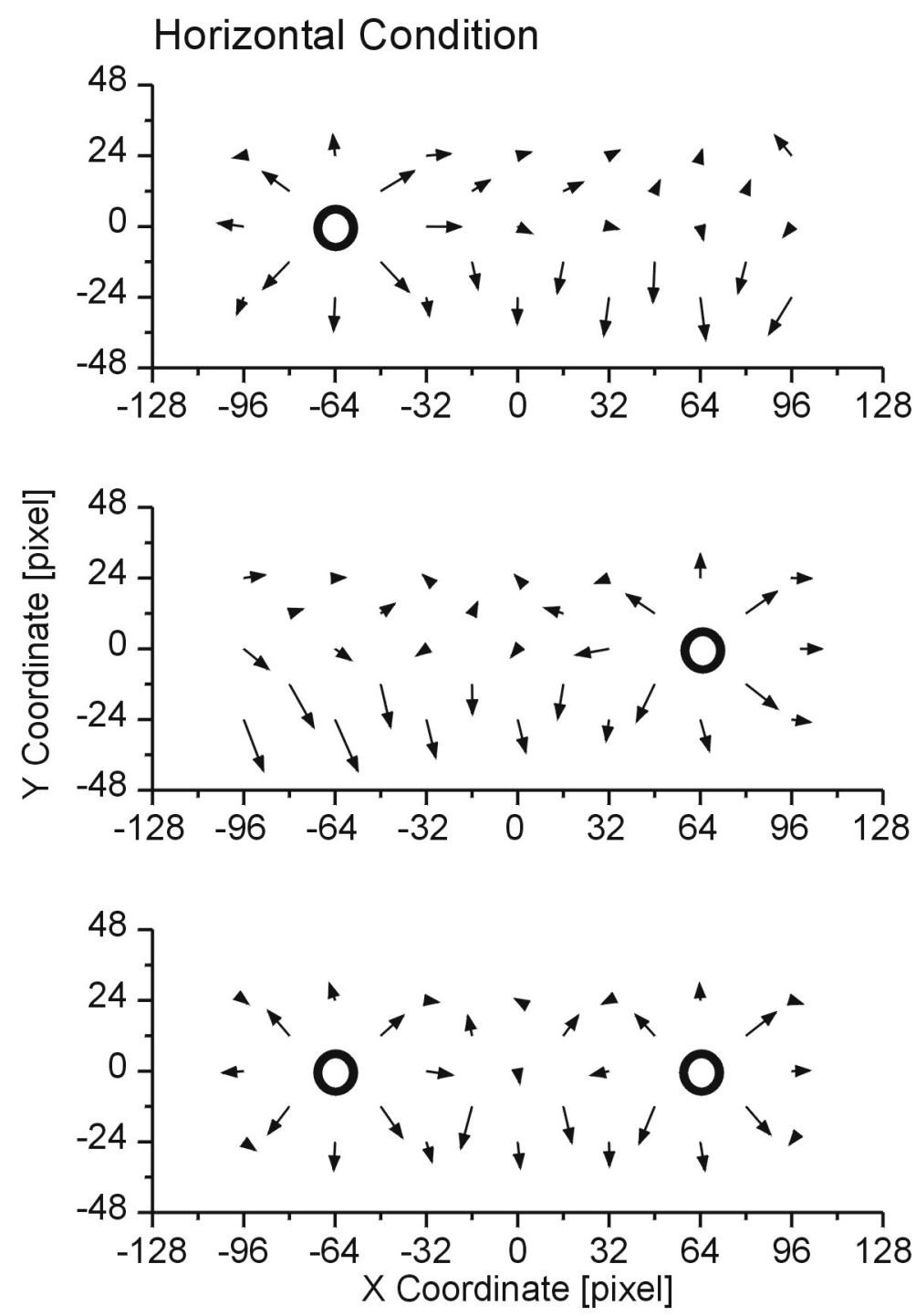

Fig. 21: Distortional fields in the horizontal condition of Experiment 6. Arrows have been magnified by a factor of 2.5 for readability. 

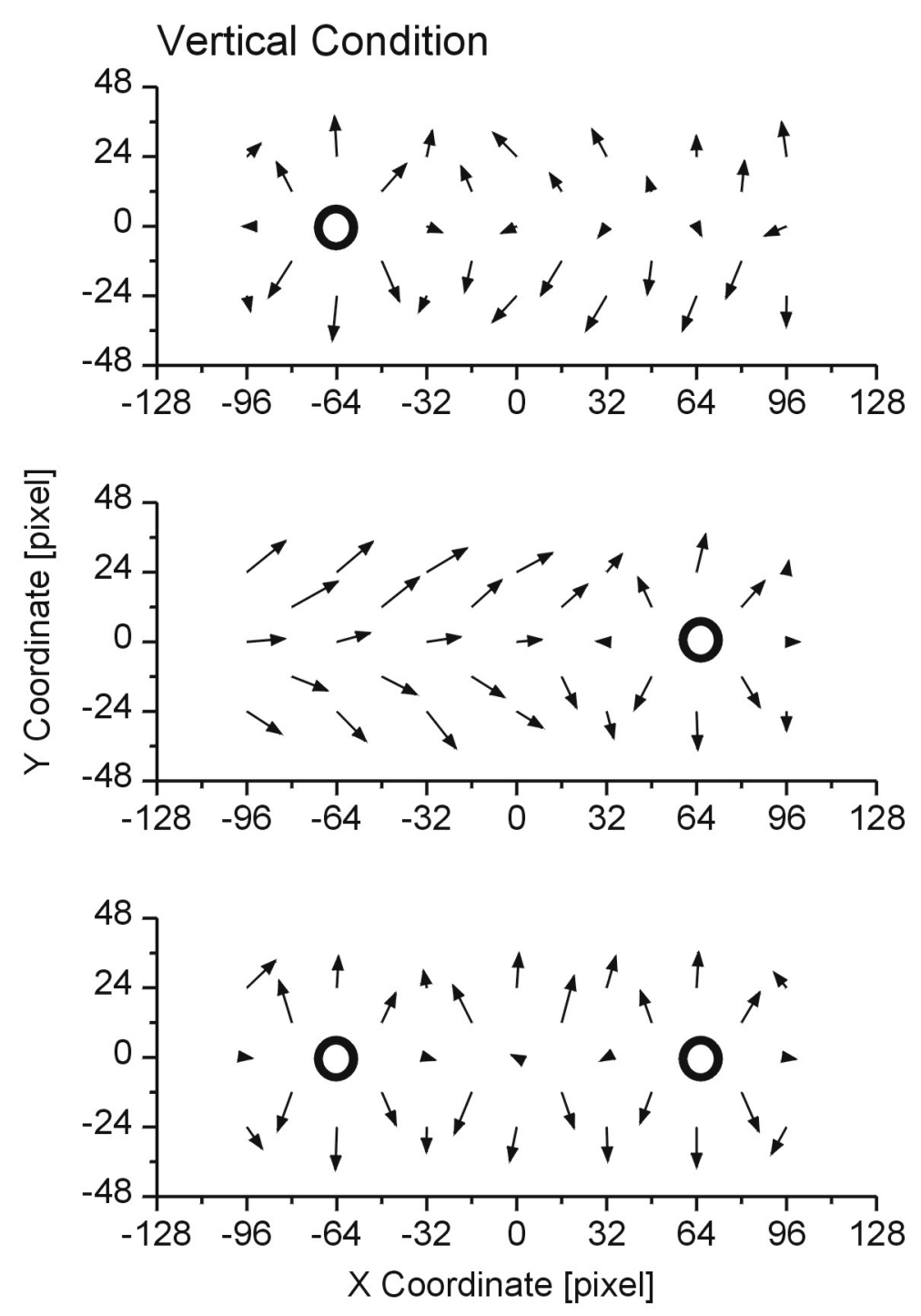

Fig. 22: Distortional fields in the vertical condition of Experiment 6 after a counterclockwise rotation by $90^{\circ}$. Arrows have been magnified by a factor of 2.5 for readability.

As observed in Experiment 5, distortional fields in the vicinity of single landmarks were very similar to the corresponding regions in the two-landmark conditions (Figs. 23, 24): biases and variances around a given landmark were the same whether or not the other landmark was present. Again, this holds for both constant and variable error and strongly suggests some partitioning model. 

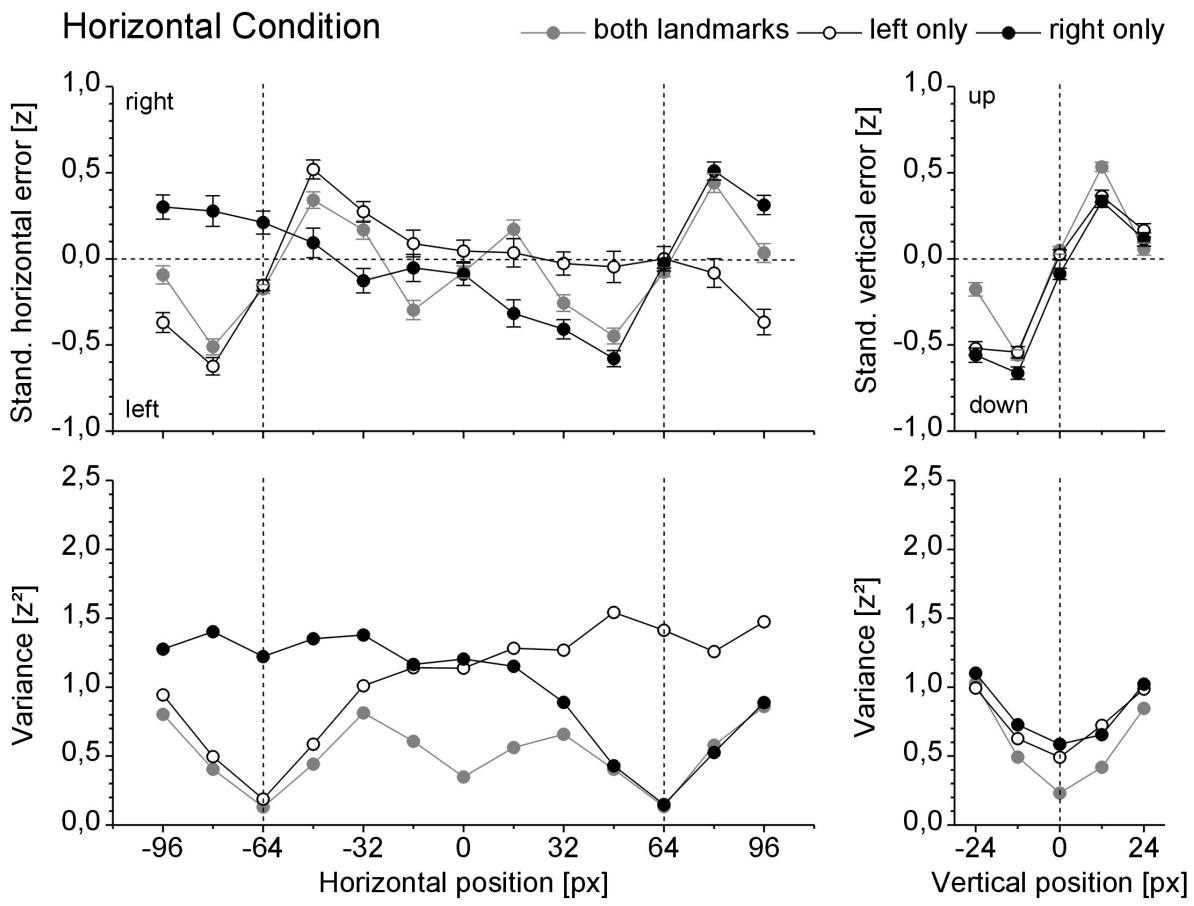

Fig. 23: Average systematic and variable error (upper vs. lower panel) in the horizontal and vertical direction (right vs. left panel) for the horizontal condition of Experiment 6 . Standard errors are between trials.

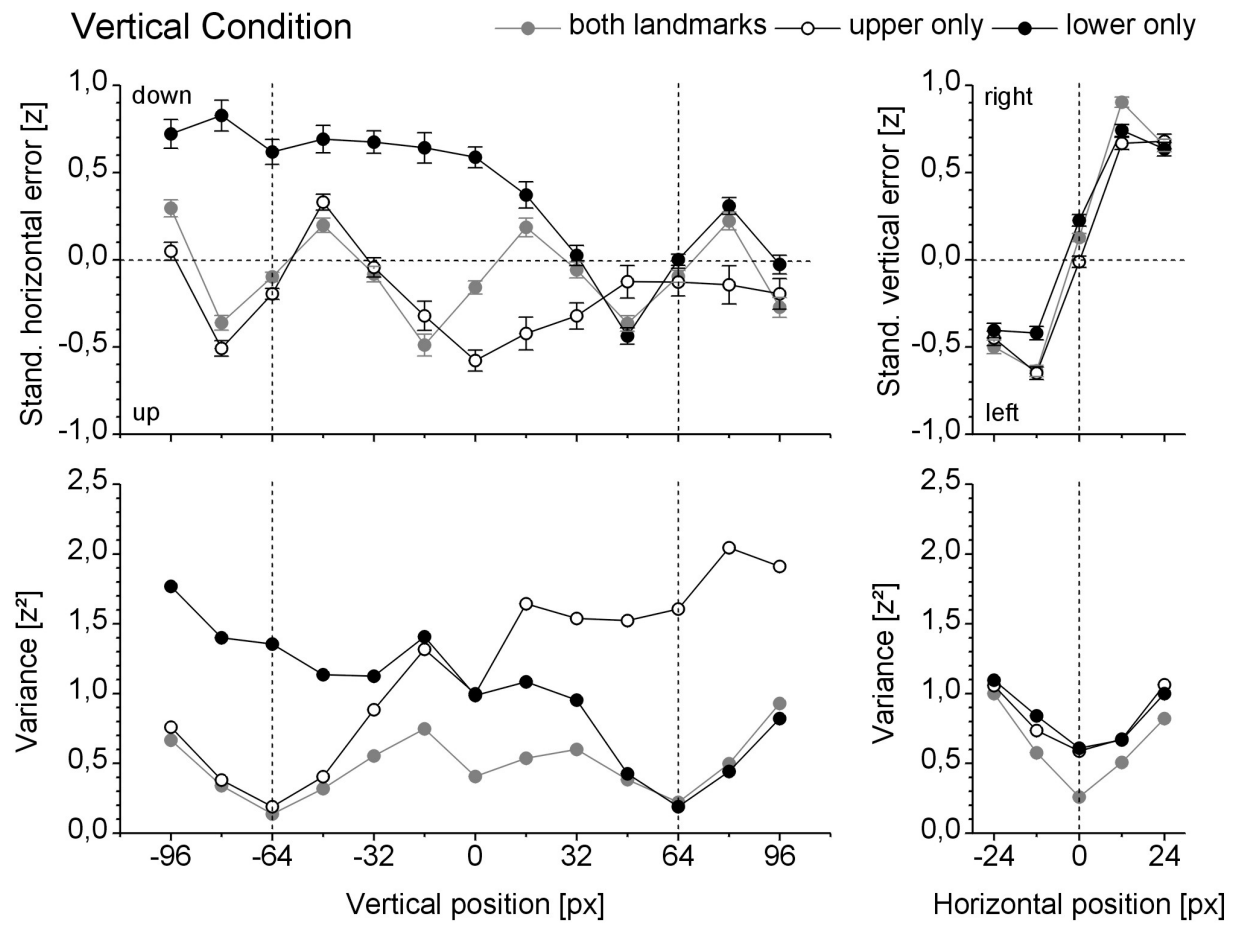

Fig. 24: Average systematic and variable error (upper vs. lower panel) in the horizontal and vertical direction (right vs. left panel) for the vertical condition of Experiment 6. Standard errors are between trials. 
For the horizontal condition, there was a main effect of target position on memory distortion, $\Lambda(60,418)=.329, p<.001$, but not of landmark condition, $\Lambda(4$, $26)=.662, p>.20$. Importantly, patterns differed across landmark conditions, as shown by a significant interaction of target position by landmark condition, $\Lambda(120$, $838)=.431, p<.001$.

In the vertical condition, there was also a main effect of target position, $\Lambda(60$, $418)=.190, p<.001$, as well as an interaction of target position by landmark condition, $\Lambda(120,838)=.565, p<.001$. However, there was also a main effect of landmark condition, $\Lambda(4,26)=.352, p<.01$. Similar to Experiment 5 , this effect was significant only in the vertical component of distortion (vertical: $F(2,14)=10.462, p<.005$; horizontal: $F(2,14)=1.224, p>.30)$ and was mainly due to the fact that the average normalized distortion was upward in the upper-landmark condition, downward in the lower-landmark condition, and neutral in the two-landmark condition.

\section{Model tests for horizontal configurations.}

As before, the two-parameter linear model was fitted to data by least-squares regression, separately for the horizontal and vertical conditions. Comparisons of observed and predicted values for the horizontal condition are shown in Fig. 25. The best-fitting linear model adds left- and right-landmark fields with weights of 0.507 and 0.427, again approximating some averaging model. This model severely underestimates the amount of horizontal error in most regions of the display and fails to predict distortion away from the midpoint.

The superposition model makes much more reasonable predictions about the pattern of biases, only slightly overestimating the amount of horizontal error in the display. However, it grossly overestimates the amount of downward error in the lower half of the display. It does not predict any distortion away from the midpoint.

Finally, the partition model predicts the amount of horizontal error about as well as does the superposition model, both making almost identical predictions. This time, it fails to account for the bias away from the midpoint. However, the superposition model allows for accurate predictions of variable error (Fig. 26). In contrast to the linear models which can make variance predictions only under strong assumptions, the partition model makes reasonable predictions about the pattern of variable error. As before, it overestimates the amount of variance near the partition line. Regression 
analysis reported in Table 2 shows that the partition model fits the data not substantially better than do the linear models.
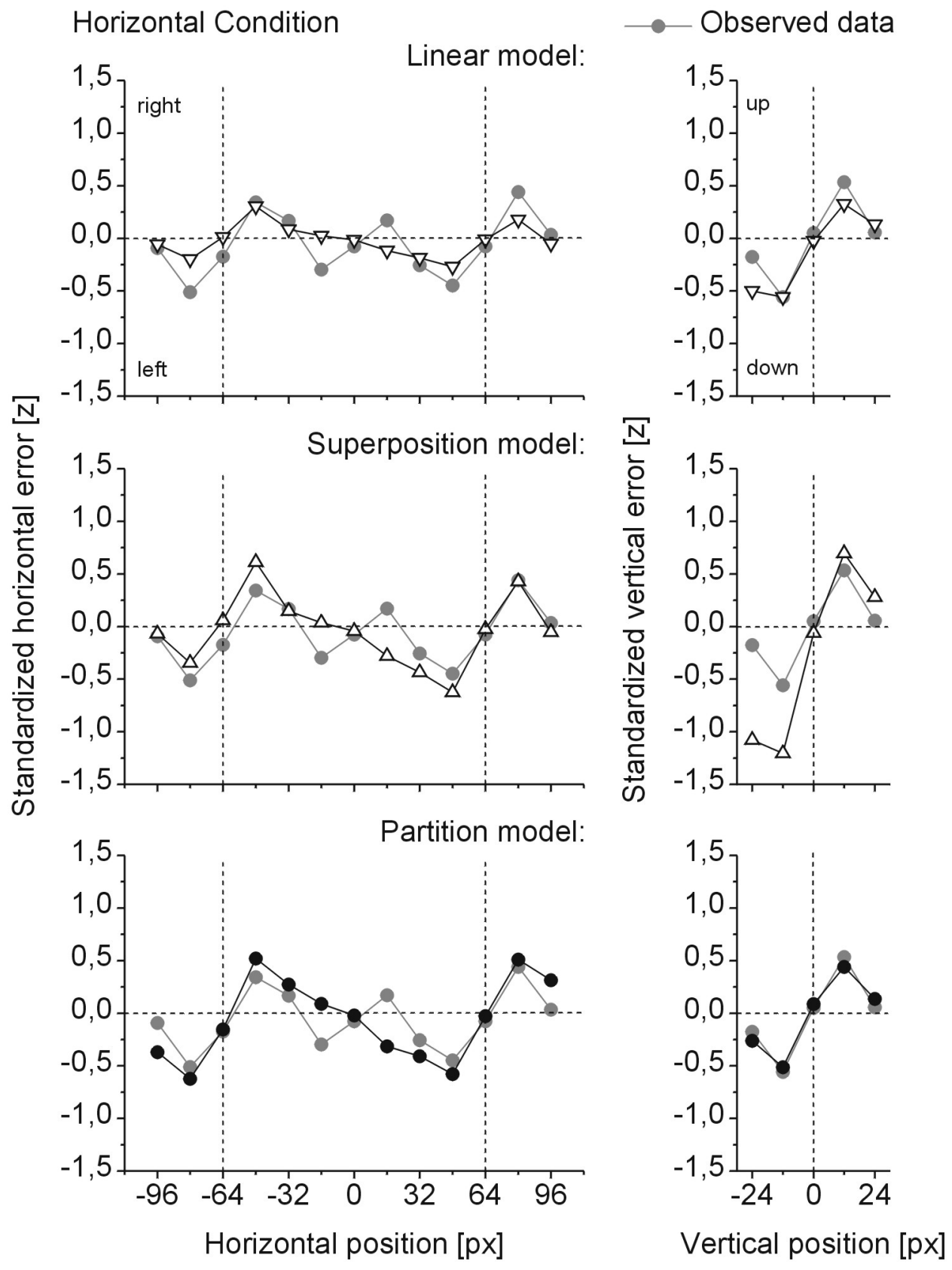

Fig. 25: Data and predictions of systematic error for the three models in the horizontal condition of Experiment 6. Horizontal error is in the left, vertical error in the right panel. 
Horizontal Condition

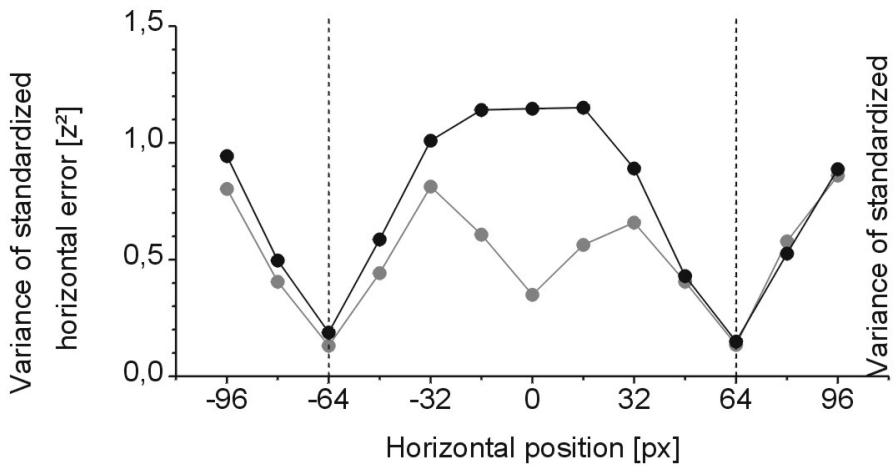

observed $\longrightarrow$ - partition model

Fig. 26: Data and predictions of variable error for the partition model in the horizontal condition of Experiment 6. Horizontal error is in the left, vertical error in the right panel.

Table 2: Goodness-of-Fit of the models in Experiment 6, horizontal condition

\begin{tabular}{|c|c|c|c|}
\hline Effect: & slope & intercept & $\mathrm{R}^{2}$ \\
\hline \multicolumn{4}{|c|}{ General Linear Model } \\
\hline Constant Error: $\mathrm{x}$ & 1.456 & -0.025 & .623 \\
\hline Constant Error: Y & 0.933 & 0.098 & .840 \\
\hline \multicolumn{4}{|l|}{ Superposition Model } \\
\hline Constant Error: $\mathrm{x}$ & 0.666 & -0.029 & .602 \\
\hline Constant Error: Y & 0.435 & 0.100 & .842 \\
\hline \multicolumn{4}{|l|}{ Partition Model } \\
\hline$\overline{\text { Constant Error: } \mathrm{x}}$ & 0.595 & -0.019 & .649 \\
\hline Constant Error: y & 1.050 & 0.005 & .961 \\
\hline Variable Error: $\mathbf{x}$ & 0.454 & 0.186 & .469 \\
\hline Variable Error: y & 1.103 & -0.085 & .815 \\
\hline
\end{tabular}

Model tests for vertical configurations.

Comparisons of observed and predicted values in the vertical condition are shown in Fig. 27. The best-fitting linear model adds upper- and lower-landmark fields with weights of 0.316 and 0.212 , respectively, again approximating some averaging model. This model severely underestimates the amount of vertical error and fails to predict distortion away from the midpoint. It also underestimates the amount of horizontal error. 

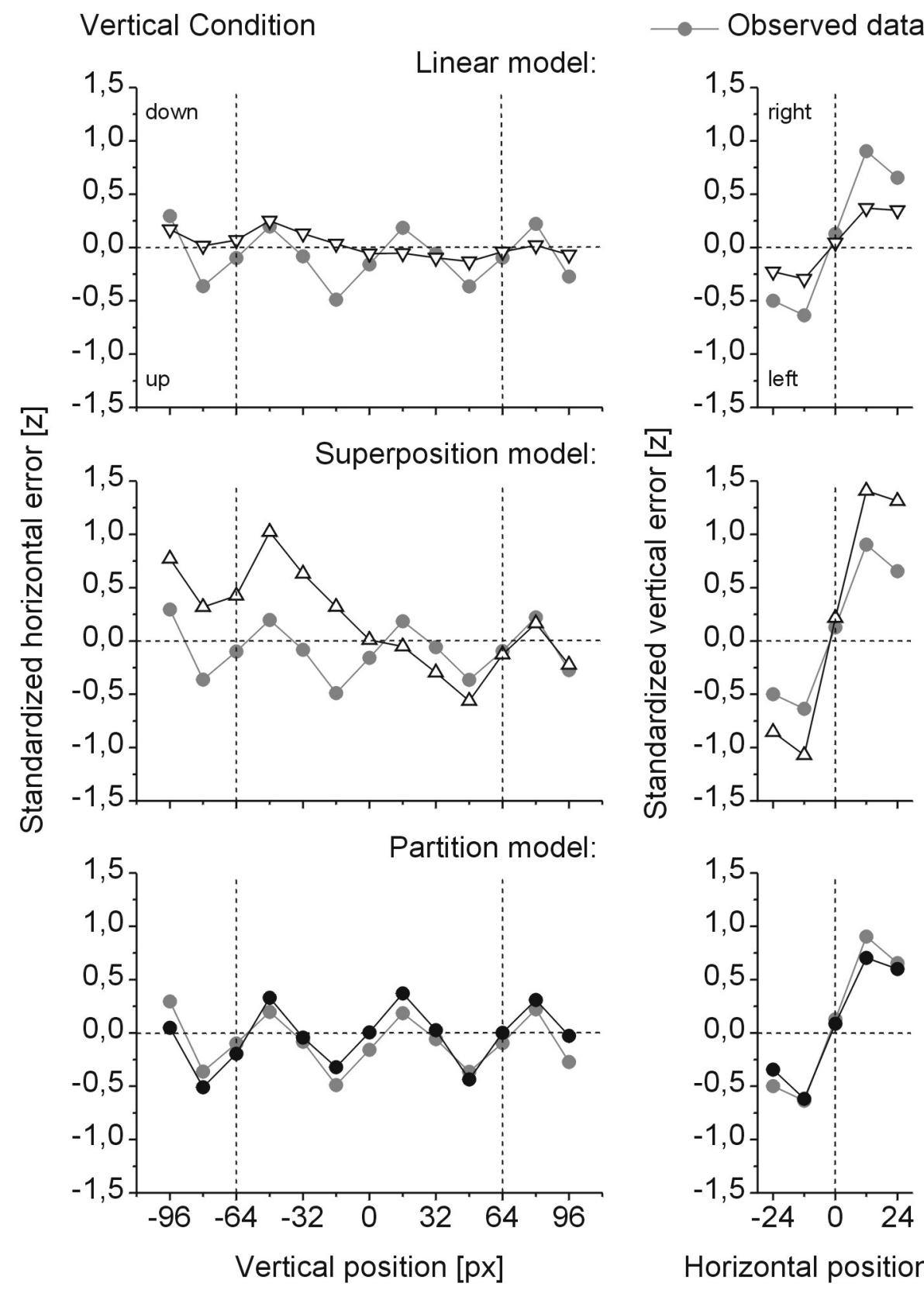

Horizontal position [px]

Fig. 27: Data and predictions of systematic error for the three models in the vertical condition of Experiment 6. Vertical error (along the longer axis of the configuration) is in the left, horizontal error in the right panel.

In contrast to its more reasonable predictions of horizontal error in the horizontal condition, the superposition model now grossly departs from the actual pattern observed in the vertical condition. This happens mainly because the large amount of vertical error in the lower-landmark condition enters the estimate, leading to overes- 
timation of vertical error in the lower half of the display. The model also overestimates the amount of vertical error and fails to account for the bias away from the midpoint.

Finally, the partition model fits the data very well. It closely predicts horizontal and vertical error, even capturing the distortion away from the display midpoint. It also makes reasonable predictions about the pattern of variable error near the landmarks (Fig. 28). Again, it overestimates variances near the partition boundary.

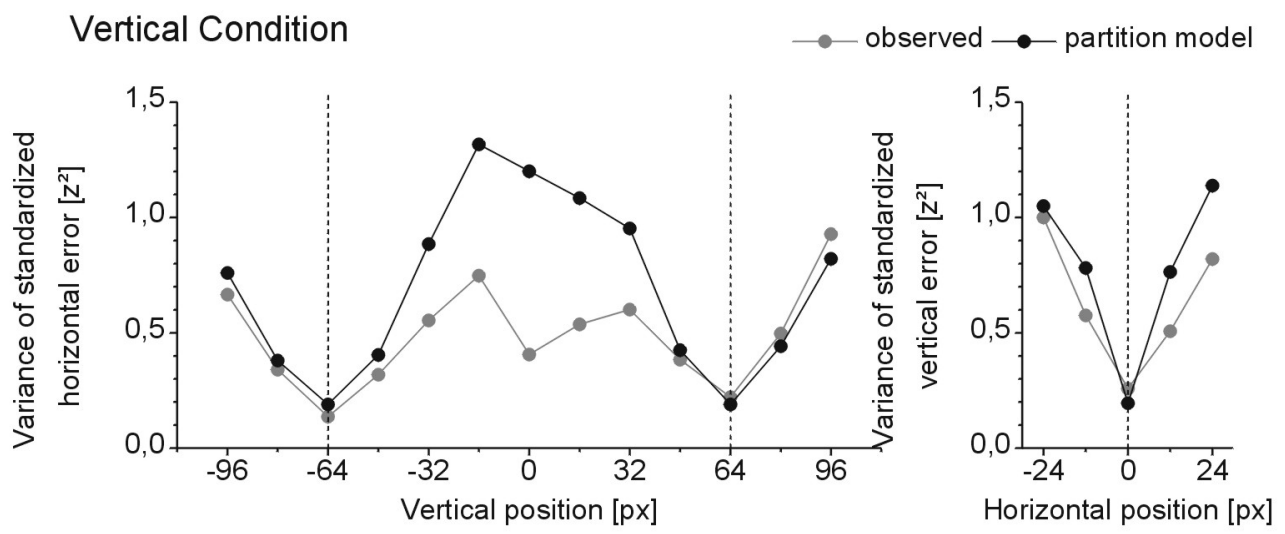

Fig. 28: Data and predictions of vertical error for the partition model in the vertical condition of Experiment 6.

Table 3: Goodness-of-Fit of the models in Experiment 6, vertical condition. Note that $\mathrm{x}$ and $\mathrm{y}$ still refer to horizontal and vertical coordinates, respectively.

\begin{tabular}{|c|c|c|c|c|}
\hline Effect: & slope & & intercept & $\underline{\mathrm{R}^{2}}$ \\
\hline \multicolumn{5}{|l|}{ General Linear Model } \\
\hline Constant Error: y & 1.063 & & -0.103 & .225 \\
\hline Constant Error: $\mathrm{x}$ & 2.178 & * & -0.006 & .988 \\
\hline \multicolumn{5}{|l|}{ Superposition Model } \\
\hline Constant Error: y & 0.236 & * & -0.127 & .184 \\
\hline Constant Error: $\mathrm{x}$ & 0.582 & * & -0.008 & .990 \\
\hline \multicolumn{5}{|l|}{ Partition Model } \\
\hline$\overline{\text { Constant Error: }} \mathrm{y}$ & 0.764 & & -0.058 & .719 \\
\hline Constant Error: $\mathrm{x}$ & 1.174 & & 0.009 & .987 \\
\hline Variable Error: y & 0.390 & * & 0.216 & .413 \\
\hline Variable Error: $\mathrm{x}$ & 0.713 & & 0.072 & .838 \\
\hline
\end{tabular}

Regression analysis reported in Table 3 shows that the partition model fits the data more closely than the other models do. Slope parameters of the two-parameter 
linear model tend to be too large while those of the superposition model tend to be too small. The most important differences can be seen in the amount of explained variance of vertical error, yielding $R^{2}$ values of .724 for the partition model but only very unsatisfactory values of .225 and .184 for the two-parameter linear and superposition models, respectively.

\subsubsection{Discussion}

Both horizontal and vertical configurations of landmarks induce a systematic pattern of distortion where reproduced target positions in the vicinity of the landmarks are biased away from the landmarks in a radial fashion. In the two-landmark conditions, there is some additional distortion away from the center between the landmarks, replicating the results from Experiment 5. However, some details of the pattern obviously depend on display orientation, at least in the single-landmark conditions. Symmetry is generally better in the vertical condition, including symmetry of the distortional field about the elongated axis of the display, resembling the fishbone-like pattern observed in Experiment 5. Symmetry is worse in the horizontal condition, obviously due to the fact that there is a general downward bias in reproductions that is especially pronounced in the lower part of the display. Such asymmetries are not discernible between the left and right halves of the field. Global downward bias is also present in the vertical condition where it leads to asymmetries between the two single-landmark patterns. This might be a result of the changed viewing conditions: in Experiment 5, the presence of an extrinsic rectangular reference system defined by the monitor frame might have helped neutralizing the downward bias and at the same time induced encoding of single-landmark fields in monitor-aligned coordinates, thereby enhancing symmetry of distortional fields. Such an interpretation is also suggested by the fact that the downward bias is eliminated when the second landmark is provided as an additional spatial reference. This point requires further investigation since these conditions can be compared only across experiments and different groups of participants here. However, remember that the inclusion of a rectangular frame in Experiment 3 seems to have helped establish rotational invariance by enhancing symmetry.

Despite its simplicity, the partition model fits the data more closely than either the two-parameter linear or the superposition model. The two-parameter linear model, though the best in terms of least-squares residuals, tends to perform some 
vector averaging operation that grossly underestimates the amount of systematic error in reproductions. In contrast, the superposition model makes reasonable predictions of constant error in the horizontal condition but inaccurate predictions in the vertical condition, mostly by adding completely different patterns of vertical error in the upper- and lower-landmark conditions.

In contrast, the data do well support the partition model. A model such as this is clearly suggested by the observed patterns in the single-landmark conditions, which are closely approximating the corresponding local patterns in the two-landmark conditions, both for constant and variable error. The only problematic region even for the partition model is the midpoint between the landmarks. The model does not consistently predict memory distortions away from this point, and it tends to overestimate variances in its vicinity.

\subsubsection{General Discussion of Experiments 5 and 6}

Experiments 5 and 6 clearly show that the distortional fields of single landmarks do not combine linearly. To show this, two linear models were tested, one superposition model stating that distortional fields of two landmarks simply add, and a more general two-parameter linear model that allows for weighting of single-landmark fields with some constants. The major weaknesses of the superposition model are that it overestimates the amount of distortion in most areas of the display, that it predicts distortion where none occurs, and that it predicts a bias towards the center position between the landmarks rather than away from it. Weighting the individual fields before summing cannot fix any of these problems because the distortions near a landmark are the same irrespective of whether or not a second landmark is present, while distortions farther away from single landmarks follow different patterns. Therefore, any single-landmark field needs a weight close to 1 in the half containing the landmark but a weight close to 0 in the opposite half. This makes plausible why fitting a linear model with both weights as free parameters leads to a very unsatisfactory averaging model which largely underestimates the distortional effects everywhere in the display. Therefore, it can not only be concluded that distortional fields fail to combine as a superposition system - they do not form any other kind of linear system either.

In marked contrast to the linear models, a simple partition model without any free parameters fits the data surprisingly well. An additional advantage of the partition 
model over the linear models is that variance predictions follow very naturally from it while the linear models yield variance predictions only under a set of strong assumptions. One of these assumption is that landmarks are the only sources of variance in the distortional field, which is proved wrong by Experiments 1 and 2 of this thesis: landmarks serve to diminish positional uncertainty, not to generate it.

However, the partition model does not consistently predict distortion away from the midpoint; in addition, it overestimates variable error at the partition boundary. Indeed, reproductions of targets located directly on the midpoint show very little scatter, seemingly forming a region of very low positional uncertainty. This strongly suggests that the distortion near the midpoint might arise from strategies of encoding the mid-position as an additional spatial reference or anchor point. The midpoint would then function like a virtual landmark, creating its own pattern of distortion. This concept of virtual landmarks is in line with a psychophysical model by Hollands and Dyre (2000) showing that when observers strategically code stimuli relative to (virtual) anchoring points on a psychophysical continuum, this creates a cyclical pattern of biases similar to the one observed here. Moreover, Bryant and Subbiah (1993) have shown that strategic differences have large effects on spatial memory distortions.

It is therefore important that adding a landmark to an existing configuration gives rise to geometrical properties that were not present before. When a second landmark is provided, not only the landmark itself can be used as a spatial reference, but also the virtual midpoint between the landmarks, the virtual line connecting them, and perhaps additional geometrical cues constructed in short-term memory. The potential relevance of such geometrical auxiliaries is nicely illustrated by Psotka (1978). Participants had the simple task of arbitrarily placing a single dot anywhere in an outline figure presented on a sheet of paper. Surprisingly, with large groups of subjects, very systematic patterns emerged depending on the geometry of the outline figure: people tended to place their dots on imaginary lines, e.g., connecting corners, lines connecting midpoints, and the perpendiculars to these lines. These findings are in general agreement with our data showing minimal departures from the virtual line connecting two landmarks and from the perpendicular one halving this line. It is also in agreement with Experiments 7 to 9 of this thesis using configurations of three landmarks. 
The results reported here are consistent with attentional models like Suzuki and Cavanagh's (1997) which assume that distortional effects are strictly local (see General Discussion for a more detailed treatment). They are not predicted by either the Nelson and Chaiklin (1980) model nor the Huttenlocher et al. (1991) model. Although Nelson and Chaiklin's model correctly predicts that distortional fields combine after being weighted with a nonlinear function that declines with increasing distance from the landmark, it does not recognize that this weighting function must be a symmetrical step function, leading to a partitioning of visual space. Worse, the model incorrectly assumes that spatial memory is biased towards a landmark, not away from it, and that the distortional effect becomes stronger with increasing distance from the landmark. With the weighting function approximating a simple step function, the decrease in the weighting function would not be able to counteract this effect near the landmarks, so that the predictions of the model are clearly falsified by the data.

Although the Huttenlocher et al. (1991) model does not make any straightforward predictions about how two landmarks combine, its prediction of spatial biases jointly depends on the geometry of the stimulus and the coordinate system used by the participants, which usually cannot be determined beforehand. Although partitioning of visual space is not predicted by this model, it makes plausible why additional distortional effects are present near the midpoint between landmarks: adding a second landmark leads to new category boundary along the vertical midline and along the line connecting the landmarks, so that the model could easily account for distortion away from the midpoint. However, some of the main assumptions of the model are inconsistent with the data. The model would explain repulsion from the midpoint by assuming a) that category membership of this point is unclear and b) that the location of the point itself is uncertain. Because a target might randomly be assigned to one or the other spatial category, memory would be unbiased in the long run but should be associated with high spatial uncertainty. The same reasoning holds for regions near the landmarks. In contrast, we find that the midpoint is a region of very low spatial uncertainty, which is just the opposite of what Huttenlocher et al.'s (1991) model predicts. 


\section{Part III: Three Landmarks}

The final part of this thesis deals with the influence of extrinsic reference systems on spatial memory distortions. Effects of extrinsic reference systems on distortional fields allow for a separation of theories supposing that distortions are exclusively determined by intrinsic properties of the landmark configuration (e.g., Nelson \& Chaiklin, 1980; Watson, 1977) and theories that allow for strategic influences in encoding spatial location (e.g., Huttenlocher et al., 1991; see also Bryant \& Subbiah, 1993).

All experiments on spatial memory distortions reported so far have worked with either one or two landmarks. Displays of this sort have special symmetry properties. For example, a space containing a single landmark is radially symmetric in the vicinity of the landmark, at least when additional spatial references like the monitor edges are ignored. Therefore, there is an infinity of possible symmetry axes. In contrast, a space containing two landmarks cannot have more than two symmetry axes, the one connecting the landmarks and the perpendicular one going through the midpoint between the landmarks.

However, the intrinsic reference systems defined by the geometry of the stimuli are probably not the only ones having some influence on distortional fields. For example, Experiment 6 suggests that spatial memory has a downward bias which gives the allocentrically vertical axis special prominence and enhances symmetry about that axis. In general, distortional fields seem to depend jointly on intrinsic and extrinsic reference systems. Although intrinsic reference systems seem to dominate in most datasets we have discussed so far, interactions between misaligned reference systems are possible. As a caveat, note that the misalignment of intrinsic and extrinsic reference systems in Experiment 3 had little or no effect on distortional fields.

In a system with only one landmark (as used in Experiments 1 and 2), there is no prominent intrinsic axis that could interact with an extrinsic frame of reference. In a system with two landmarks (as used in Experiments 3 to 6), both possible intrinsic axes are perpendicular to each other. Because most extrinsic reference systems (like monitor frames, room edges, gravity) also consist of perpendicular axes, alignment or misalignment with the extrinsic system holds for both axes simultaneously. Therefore, it is not possible to compare an aligned intrinsic axis with a misaligned intrinsic axis in the same vector field. 
In order to make such a comparison, one needs a system with an uneven number of landmarks. For example, in an equilateral configuration of three landmarks, there are three axes of symmetry forming angles of $120^{\circ}$ with each other. However, only one of these axes can be aligned with any extrinsic axis; the other two are necessarily misaligned. In the final set of experiments reported here, triangular configurations of landmarks are used to study spatial interactions of intrinsic and extrinsic reference systems. The first two experiments investigate the case where only one axis is aligned with the allocentric vertical (Experiment 7 ) or where all three axes are misaligned (Experiment 8). The third experiment investigates possible interactions of extrinsic and intrinsic reference axes by using elongated triangles where the axis of elongation does or does not coincide with the allocentric vertical (Experiment 9).

\subsection{Experiment 7}

In this experiment, two equilateral configurations of three landmarks were used. Because equilateral triangles have three axes of symmetry, distortions in visual memory might develop around any of these axes. If only intrinsic properties of the stimulus were determining distortions, the distortional field would have three equally strong axes of symmetry. In contrast, if extrinsic reference systems also influenced the distortional pattern, an intrinsic axis aligned with an extrinsic axis should display better symmetry properties. In the present experiment, triangular configurations were displayed in either top-up $\left(0^{\circ}\right)$ or top-down $\left(180^{\circ}\right)$ orientation so that one of their symmetry axes was always aligned with the allocentric vertical. It was expected that symmetry about this axis should be stronger if extrinsic references contributed to the distortional effect.

\subsubsection{Method}

\section{Participants.}

Eight students (age 15 to 40, one male, all right-handed) of the Institute of Psychology at the University of Göttingen participated for course credits or for a payment of 15,- DM per hour. Their vision was normal or corrected-to-normal. 


\section{Stimuli.}

The apparatus was as described in Experiment 6. All stimuli were presented against a black background $\left(0.01 \mathrm{~cd} / \mathrm{m}^{2}\right)$. Landmarks were three green unfilled circles $\left(28.7 \mathrm{~cd} / \mathrm{m}^{2}\right), 9 \mathrm{px}\left(0.26^{\circ}\right)$ in diameter, that were arranged in an equilateral triangle with a side length of $180 \mathrm{px}\left(5.20^{\circ}\right)$, its center of mass located at the screen center. This configuration could appear either top-up $\left(0^{\circ}\right.$ orientation) or top-down $\left(180^{\circ}\right.$ orientation). The target was a small white dot $\left(43.0 \mathrm{~cd} / \mathrm{m}^{2}\right)$ with a diameter of $3 \mathrm{px}$ $\left(0.09^{\circ}\right)$, presented at one of the 41 locations shown in Fig. 29. A dynamical mask was used as described in Experiment 1.

a) Possible landmark and target locations.
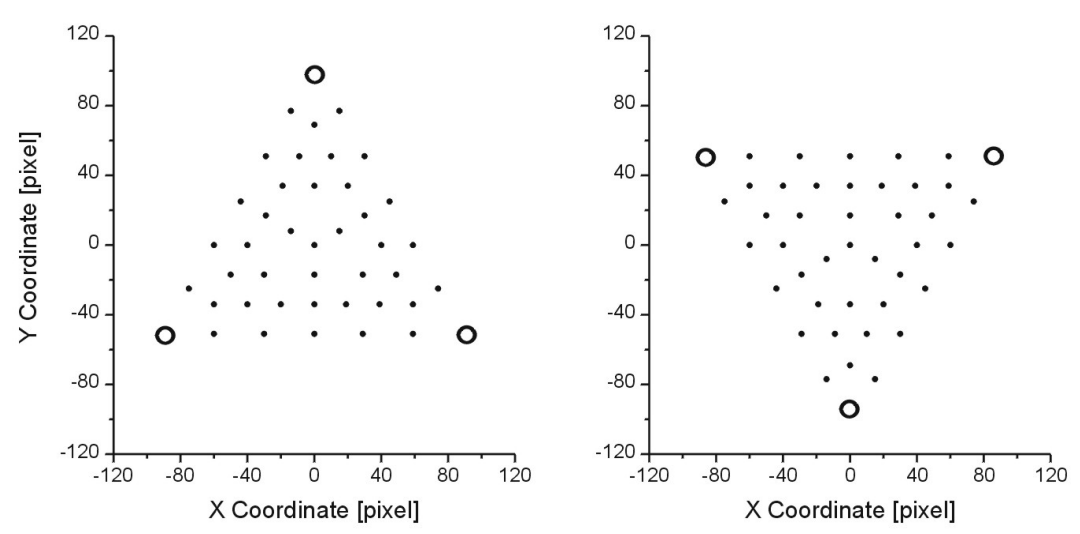

b) Time course of a trial.

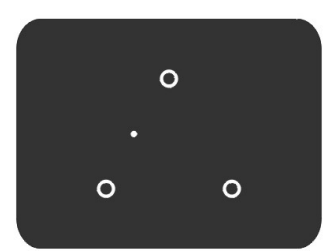

Target with three landmarks: $500 \mathrm{~ms}$

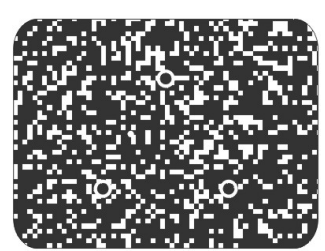

Dynamical mask (landmarks still visible): $500 \mathrm{~ms}$

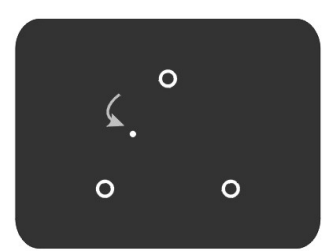

Reproduction of target location with mouse cursor

Fig. 29a: Landmarks and possible target locations in the two conditions of Experiment 7. b: Time course of a trial. 


\section{Procedure.}

A trial began with the presentation of the target together with the three landmarks for $500 \mathrm{~ms}$. The target was then replaced by the dynamic mask for $500 \mathrm{~ms}$ while the landmarks remained visible. Immediately after the masking interval, a mouse cursor looking exactly like the target appeared randomly in one of the landmark locations. After the participant had reproduced the target position, a $1000 \mathrm{~Hz}$, $100 \mathrm{~ms}$ tone was sounded for feedback that the response had been registered. After an intertrial interval of $500 \mathrm{~ms}$, a new trial began. Participants were instructed to work quickly, but it was emphasized that accuracy rather than speed was important in this task.

Stimulus conditions were counterbalanced such that each combination of orientation $\left(0^{\circ}, 180^{\circ}\right)$, starting position of the mouse cursor, and target position occured quasi-randomly and equiprobably, with each combination appearing once every 12 blocks. After each block, participants received summary feedback about their average euclidean deviation from the true target position, rounded to the nearest pixel. Participants performed one session of 20 blocks with 24 trials each. Each session started with an additional practice block of 24 trials with stimuli drawn randomly from the experimental blocks. Practice trials were not analyzed. After the final session, participants were debriefed and received an explanation of the purpose of the experiment.

\section{Data treatment.}

Trials with reponse times faster than $100 \mathrm{~ms}$ or slower than $10.000 \mathrm{~ms}$ were excluded because these trials likely reflected anticipatory or guessing behavior. Trials with a euclidean target deviation larger than $30 \mathrm{px}$ were also excluded. The whole procedure eliminated $1.35 \%$ of the raw data.

\subsubsection{Results}

Overall effects.

Vector fields of memory distortions were first normalized by rotating them into a $0^{\circ}$ orientation, and then analyzed by a MANOVA design with target position and triangle orientation as fixed factors and participants as a random factor, using the $x$ (horizontal) and $y$ components of distortion as dependent variables. Landmarks indu- 
ced a complex but regular pattern of distortions, which was qualitatively similar for both display orientations (Fig. 30). Distortional effects along the imaginary sides of the triangles were qualitatively similar to those observed along the horizontal axes connecting two landmarks in Experiments 3, 5, and 6, with distortion away from the landmarks, away from the midpoints of the triangle sides, and away from the midpoints. Again, there was an effect of repulsion near the landmark that turned into attraction at some distance from it. Finally, there was also some distortion away from the center of gravity of the entire figure, which was more pronounced in the $0^{\circ}$ condition. Importantly, the pattern of distortions seems to respect major lines and axes in the three-landmark figure: there is little departure from the virtual sides of the triangle or from the vertical mid-axis.
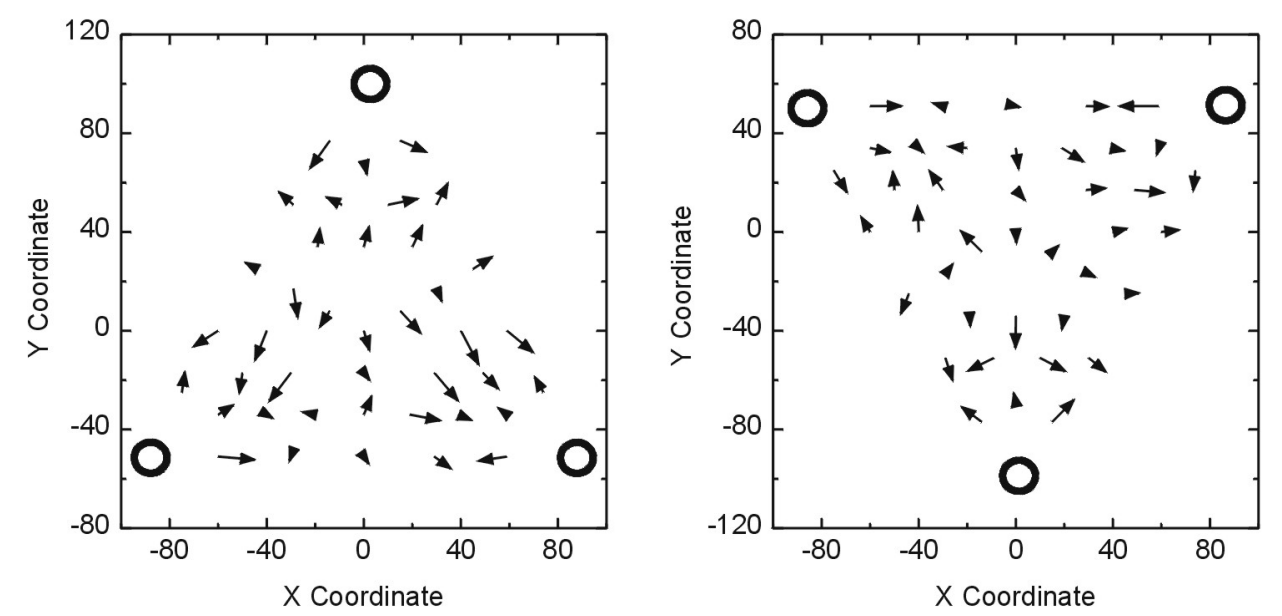

Fig. 30: Distortional fields in Experiment 7. Arrows are magnified by a factor of 2.5 for readability.

MANOVA confirmed that the vectors of distortion depended on target position, $\Lambda(78,544)=0.307, p<.001$. This effect was significant for both the $x$ and $y$ components of distortion, $F(39,273)=6.708$ and $4.662, M S E=58.753$ and 74.286 , both $p$ $<.001$. There was no main effect of landmark orientation, $\Lambda(2,6)=0.723, p>.37$, indicating that the net vectors of distortion (averaged over all target positions) were comparable in the 0 and $180^{\circ}$ conditions. Importantly, there was no interaction of both factors, $\Lambda(78,544)=0.753, p>.34$, showing that both vector fields did not differ systematically. 
Two measures of symmetry.

Symmetry in vector patterns was evaluated in a two-fold strategy: first, by using correlations between opposite halves of the vector fields, and second, by deriving a descriptive measure of symmetry.

For computation of correlations, the vector field was rotated so that the symmetry axis of current interest was in an upright position. First, targets lying along the symmetry axis were excluded. Then, coordinates of corresponding targets from both halves of the vector field were correlated separately for horizontal and vertical coordinates, yielding one correlation coefficient for each coordinate. Symmetry about other axes was evaluated in the same way after appropriate rotation of the pattern. With this measure, perfect symmetry would result in correlations of $r_{x x^{\prime}}=-1$ (for $x$ coordinates) and $r_{y y^{\prime}}=1$ (for $y$ coordinates). Note that this measure is invariant with respect to changes in scale between the two halves: if all vectors, say, in the right half were twice as long but had the same direction, correlations would not change. For statistical tests, correlations were compared using Fisher's $Z$ transformation with appropriate Bonferroni adjustment for planned comparisons.

A second measure of symmetry was derived by calculating the average euclidean departure from symmetry. Again, vectors lying along the symmetry axis were discarded. Next, vectors on one side of the symmetry axis were reflected upon corresponding vectors on the other side of the axis, and the difference between the two vectors was computed by vector subtraction, yielding symmetry residual vectors. The length of these vectors, averaged across all vector pairs, is an index of symmetry that is 0 if symmetry is perfect and has some finite value if asymmetry is present. Note that this symmetry index serves for purely descriptive purposes here because it is used only to quantify symmetry within vector fields that are already averaged across participants, so that confidence limits for this measure cannot be derived. 1

The results from the correlational approach are shown in Fig. 31. For both orientations, there was clear symmetry about all possible axes, all $r>.498$, all $p<.05$. In the $0^{\circ}$ condition, symmetry was significantly better for the vertical symmetry axis than about the $120^{\circ}$ axis $(z=-2.239, p<.05$ for the horizontal, $z=1.019, p>.15$ for the vertical component), and also significantly better than about the $240^{\circ}$ axis $(z=$

\footnotetext{
${ }^{1}$ Although it is possible to compute a symmetry index for each participant, the increase in statistical noise would cause the index to shift away from zero, so that values from different conditions would tend to become very similar. In particular, note that the average of symmetry indices computed for individual participants would not
} 
2.295, $p<.05$ for the vertical, $z=-.931, p>.15$ for the horizontal component). The two oblique axes did not differ significantly. In the $180^{\circ}$ condition, although symmetry about the vertical axis seems slightly better than along the oblique axes, there were no significant differences, $-0.727 \leq z \leq 0.787$, all $p>$.20. Symmetry indices give a similar picture: in both landmark configurations, the vertical axis has a lower index of symmetry than the remaining axes (Fig. 32), even though this difference is more pronounced for the $0^{\circ}$ configuration.
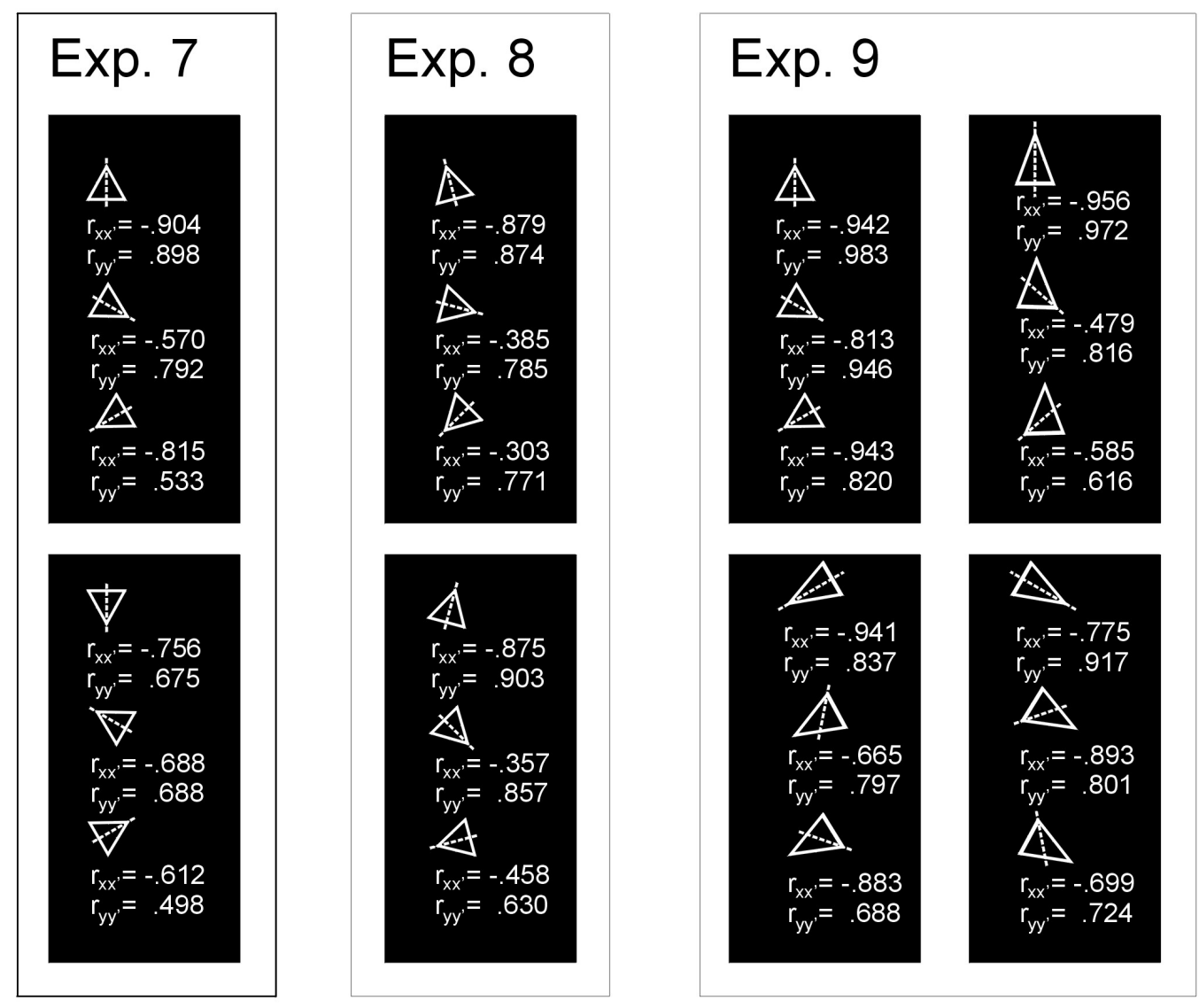

Fig. 31: Correlational symmetry tests for Experiments 7-9. Inlays depict the configuration with the symmetry axis of interest, and correlations are between corresponding vectors of distortion to the sides of the symmetry axis. Note that because of mirror symmetry, $\mathrm{r}_{\mathrm{xx}}$ (error coordinates perpendicular to the symmetry axis) should be negative while $r_{y y}$ (error coordinates parallel to the symmetry axis) should be positive. See Results section of Experiment 9 for details on symmetry computation in that dataset. 

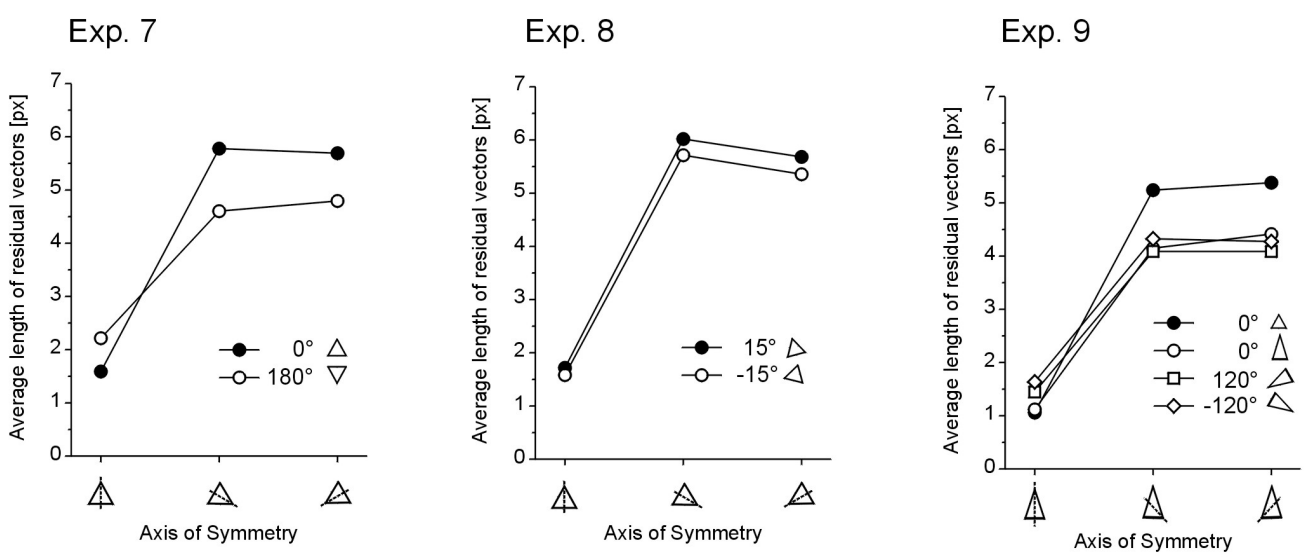

Fig. 32: Symmetry indices for Experiments 7-9. The abscissa describes the orientation of the symmetry axis of interest for a vector field normalized by rotation so that all configurations are oriented top-up. The legend captions then describe the original orientation of the configuration. See Results section of Experiment 9 for special details of symmetry computation in that dataset.

\subsubsection{Discussion}

Three landmarks arranged in an equilateral triangle induce a complex but systematic pattern of distortions in short-term visual memory that is consistent with the distortional effects observed in the more simple one- or two-landmark situations. In the vicinity of landmarks, there is some repulsion away from them, turning into attraction at some distance; there is also repulsion from the center of gravity of the triangle. All this suggests a staightforward generalization of the patterns reported before. The pattern of distortions seems strictly dependent on intrinsic stimulus properties, respecting major lines and axes in the figure and showing little dependence on its orientation. However, the superior symmetry properties of the vertical axes clearly show that extrinsic reference systems also contribute to spatial memory distortions. Based on intrinsic stimulus geometry alone, there would be no reason to expect the vertical symmetry axis to display stronger symmetry than others. These findings run counter to theories assuming that spatial distortions are exclusively stimulus-driven (Nelson \& Chaiklin, 1980; Watson, 1977).

It would be tempting to conclude that the vertical axis induces stronger symmetry because it coincides with a major axis of any of several possible extrinsic reference systems, like the body axis or gravity. In this view, alignment with a major extrinsic reference system would be sufficient for an intrinsic axis to become a strong axis of symmetry. However, it is possible that this axis becomes special only by virtue 
of the attentional state of the observer cognitively coding it as especially salient because of its alignment with an extrinsic frame of reference. In this view, alignment of the axis with an extrinsic reference system would not enhance symmetry if observers were not explicitly noticing this relationship. Support for this attentional view comes from experiments by Palmer and Bucher (1981, 1982; Sekuler, 1996) who asked participants to indicate the perceived pointing direction of equilateral triangles. Because these stimuli are inherently tri-stable, participants reported attentional switching between percepts. However, aligning one of the triangle's axes with a rectangular frame induced a strong bias to perceive the triangle pointing into the direction induced by the extrinsic reference frame.

Experiment 8 was designed as a test whether the results obtained here would generalize to an equilateral triangle of landmarks where neither axis coincided with any extrinsic reference axis. It also investigated the idea that symmetry properties of the vertical axis reflected perceptual and attentional salience rather than an automatic effect of vertical alignment.

\subsection{Experiment 8}

In Experiment 7, one intrinsic axis of the triangular configuration of landmarks was always aligned with the allocentric vertical. It was found that this axis had better symmetry properties than the two oblique axes, illustrating an interaction of intrinsic and extrinsic reference systems. In Experiment 8, stimulus configurations were used where neither of the intrinsic axes were aligned with any allocentric axis. This accomplished by rotating the stimulus configuration from Experiment 7 by $15^{\circ}$ to the left or right. If alignment with the allocentric vertical automatically induced stronger symmetry properties of this axis, this manipulation should make the symmetry properties of the three intrinsic axes more comparable. In contrast, if any one axis still displayed stronger symmetry, this would argue against a purely automatic account and would endorse an attentional explanation (Palmer \& Bucher, 1981, 1982). 


\subsubsection{Method}

\section{Participants.}

Eight students (age 18 to 31, three of them male, all right-handed or ambidextrous) of the Institute of Psychology at the University of Göttingen participated for course credit or for a payment of 15,- DM per hour. Their vision was normal or corrected-to-normal.

\section{Stimuli and Procedure.}

Methods were identical to those used in Experiment 7, with the one exception that the triangular configuration of landmarks was presented in orientations of $+15^{\circ}$ or $-15^{\circ}$ (counterclockwise) instead of $0^{\circ}$ and $180^{\circ}$ (Fig. 33a).

a) Possible landmark and target locations.
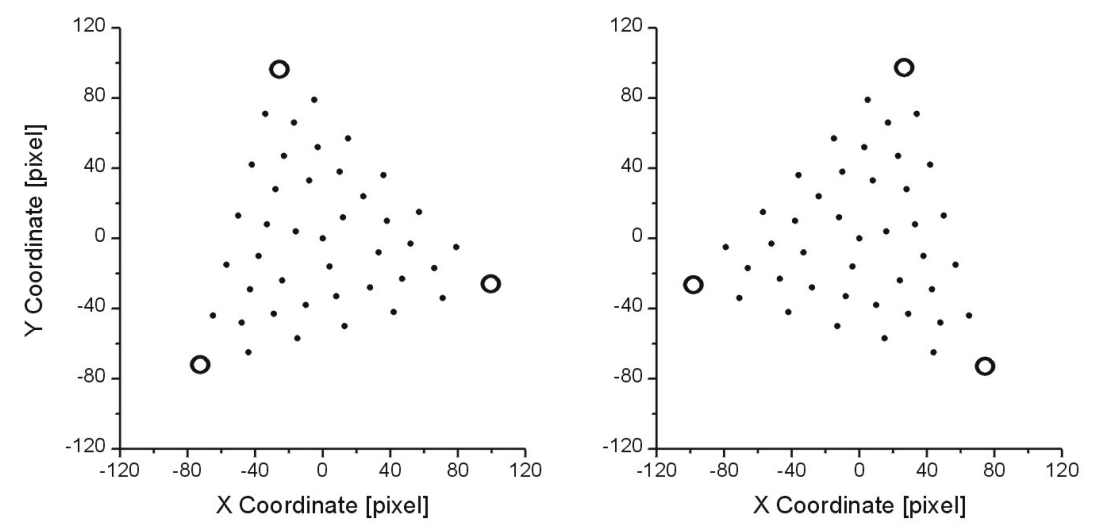

b) Time course of a trial.

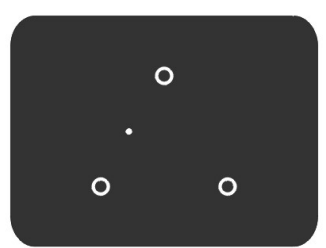

Target with three landmarks: $500 \mathrm{~ms}$

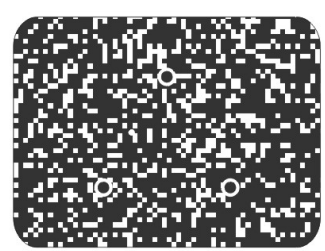

Dynamical mask (landmarks still visible): $500 \mathrm{~ms}$

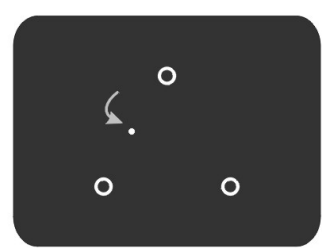

Reproduction of target location with mouse cursor

Fig. 33a: Landmarks and possible target locations in the two conditions of Experiment 8. $b$ : Time course of a trial. 
Data treatment and statistical methods.

Criteria for outlier elimination were as described in Experiment 7. The procedure eliminated $0.68 \%$ of the raw data. Statistical analysis proceeded as described in Experiment 7, after normalizing the vector fields in both orientations by rotating them into an upright $\left(0^{\circ}\right)$ position.

\subsubsection{Results}

Overall effects.

Vector fields of memory distortions were qualitatively similar to those observed in Experiment 7 (Fig. 34). In both the $+15^{\circ}$ and $-15^{\circ}$ orientation, there was distortion away from the landmarks turning to attraction towards the landmark at some distance. Again, there was distortion away from the center of gravity of the figure.

MANOVA confirmed that the vectors of distortion depended on target position, $\Lambda(78,544)=0.388, \mathrm{p}<.001$. There was no main effect of landmark orientation, $\Lambda(2$, $6)=0.852, p>.61$, indicating that the net vectors of distortion (averaged over all target positions) were comparable across conditions. However, there was a significant interaction of target position and landmark orientation, $\Lambda(78,544)=0.669, p>.005$, implying some difference in vector fields after normalization, although it is hard to tell the differences by inspecting the figures.
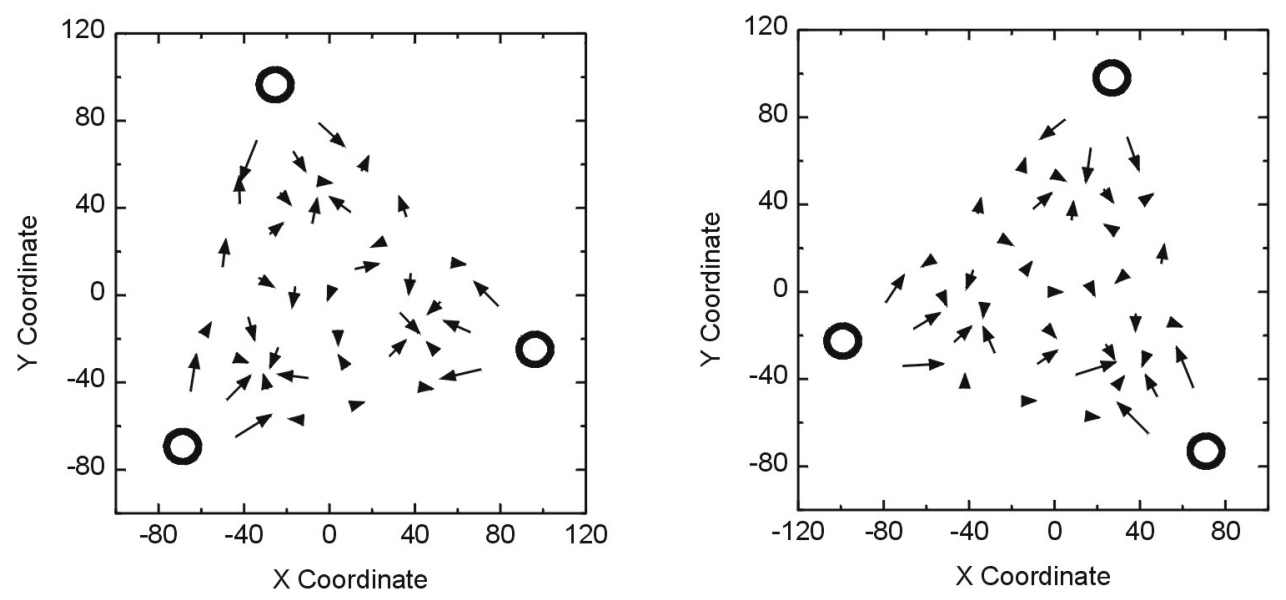

Fig. 34: Distortional fields in Experiment 8. Arrows are magnified by a factor of 2.5 for readability. 


\section{Symmetry properties.}

Symmetry in vector fields was evaluated using the methods described in Experiment 7. The results from the correlational approach are shown in Fig. 31. Symmetry was somewhat weaker than in Experiment 7. Although there were significant correlations about all axes of symmetry for the vertical coordinates, all $r \geq .630$, all $p$ $<.005$, symmetry for the horizontal coordinates was significant only about the nearvertical axes (both $p<.001$ ), but less reliable about the other axes, $-.303 \leq r \leq-.458$, $.032 \leq p \leq .119$. In the $15^{\circ}$ condition, symmetry was significantly better for the nearvertical $15^{\circ}$ symmetry axis than about the $135^{\circ}$ axis $(z=-2.554, p<.01$ for the horizontal component, $z=0.771, p>.20$ for the vertical component), and also significantly better than about the $255^{\circ}$ axis $(z=2.801, p<.005$ for the horizontal component, $z=-0.865, p>.19$ for the vertical component). The two oblique axes did not differ significantly. In the $-15^{\circ}$ condition, there was a similar pattern, with better symmetry about the near-vertical $-15^{\circ}$ axis than about the $105^{\circ}$ axis $(z=-2.594, p<.005$ for the horizontal component, $z=0.546, p>.29$ for the vertical component), and also better symmetry than about the $225^{\circ}$ axis $(z=-2.273, p<.05$ for the horizontal component, $z=1.976, p<.05$ for the vertical component). Again, the two oblique axes did not differ significantly. Symmetry indices agreed with the correlational approach: symmetry residuals were small for the near-vertical axes; residuals for the two remaing axes were about equally large and much larger than for the near-vertical; and there was no dependence on the orientation of the triangle (Fig. 32).

\subsubsection{Discussion}

The pattern of spatial memory distortions obtained in this experiment is qualitatively very similar to the one reported in Experiment 7. As before, the pattern of distortions respected major lines in the figure, with little deviations from the virtual sides of the triangles. There was a clear effect of distortion away from the landmarks as well as from the center of gravity The overall pattern of distortion seems grossly independent of the orientation of the landmark configuration.

If alignment with an extrinsic system of reference automatically strengthened the symmetry properties of an intrinsic stimulus axis, symmetry should be similar about all possible axes when they are all misaligned with the extrinsic reference. 
However, symmetry about the near-vertical axis was clearly much stronger than about the remaining two axes, suggesting that this line was used as a spatial reference even though it was not aligned with the allocentric vertical. In contrast, the nearhorizontal axis did not display enhanced symmetry properties despite its being misaligned from a major allocentric axis by the same angle. In principle, there are two possible explanations for that. First, the visual system might automatically treat the near-vertical axis as aligned with the allocentric vertical, which would imply an area of tolerance that would encompass at least $30^{\circ}$. Although there is no direct evidence against this possibilty, this degree of indifference to misalignments seems very large compared to the high precision of coordinate frame transformations that occur elsewhere in the visual and motor systems (Flanders, Helms Tillery, \& Soechting, 1992).

Second, the importance given to the near-vertical axis of symmetry may come about not by mere indifference to misalignment but by active attentional processes selecting one axis and giving it special prominence as a spatial reference. This explanation is in line with the results of Palmer and Bucher $(1981,1982)$ showing that observers can be induced to perceive any symmetry axis in an equilateral triangle as the direction of pointing of the triangle when appropriate attentional set is established. In this view, participants would attentionally select the near-vertical axis of symmetry and deliberately use it to determine, e.g., the left and right of the figure. That people might adopt object-based frames of spatial references even when the object is oriented obliquely is demonstrated by object-based neglect shown by some patients with damage to their right posterior-parietal lobule (Halligan \& Marshall, 1994), a condition that almost certainly involves disorders of spatial attention.

\subsection{Experiment 9}

In Experiments 7 and 8, only equilateral triangular configurations of landmarks were used, and the effect of alignment or misalignment with the allocentric vertical was studied. Such stimuli have three possible axes of symmetry, and are therefore ambiguous with regard to their apparent orientation. In contrast, more general configurations of three landmarks have a definite form and orientation. In Experiment 9, elongated equilateral triangles were used where the axis of elongation was also the only axis of symmetry, thereby defining a cardinal axis in an unambiguous object- 
centered, intrinsic frame of reference. Of course, this frame could be either aligned or misaligned with an extrinsic system of reference, like the one defined by gravity.

An optimal experimental strategy would be to put different orientations of intrinsic and extrinsic reference systems into a factorial design where both variables could be manipulated independently. However, many potential sources of external reference, gravity for instance, are difficult to manipulate. The direction of gravitational forces applying to an observer's body can be altered only with great difficulty by placing the observer in an accelerating device that will somehow counteract the effects of gravity, like a spacecraft in orbit (note, however, that even in a space shuttle astronauts are "weightless" only within a shuttle-centered system of reference, as gravity continuous to propel both the shuttle and the astronauts around the planet). Even if neutralization of gravitational forces is accomplished, however, additional problems can accrue because other extrinsic reference systems may now be misaligned with gravity, which also has an influence on spatial memory (Finkelmeyer, 2001).

It was therefore attempted to establish appropriate controls for an experiment where allocentric reference systems remained unchanged. The control condition was an equilateral configuration of landmarks oriented top-up on a computer display. Elongated equiscleral triangles were created by shifting the location of only one of these landmarks along a line perpendicular to the line connecting the remaining two landmarks. This construction led to four stimulus configurations whose symmetry properties could be compared, depicted in Fig. 36.

\subsubsection{Method}

\section{Participants.}

Nine students (age 19 to 37 , four of them male, one left-handed) of the Institute of Psychology at the University of Göttingen participated for course credits or for a payment of 15,- DM per hour. Their vision was normal or corrected-to-normal. 
a) Possible landmark and target locations.
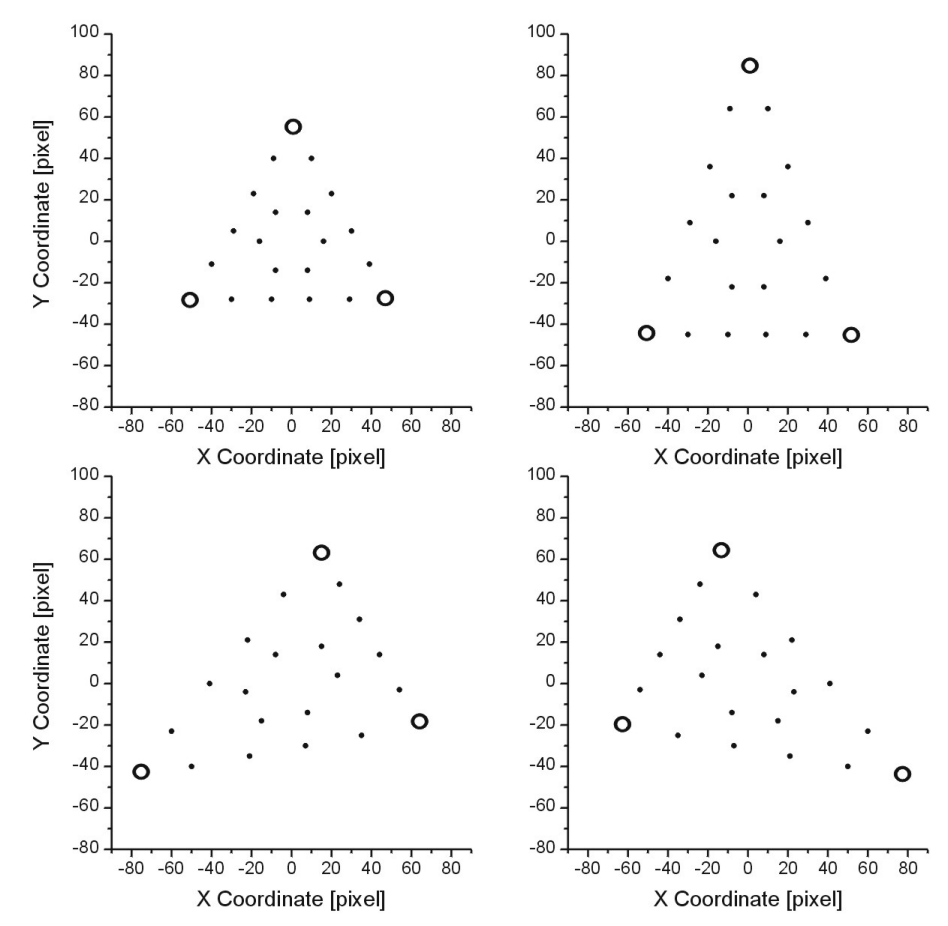

b) Time course of a trial.

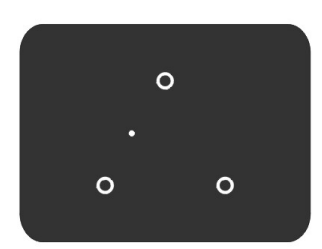

Target with three landmarks: $500 \mathrm{~ms}$

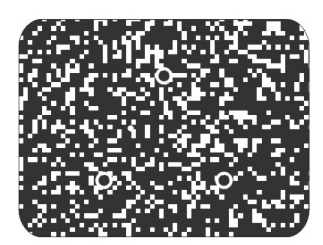

Dynamical mask (landmarks still visible): $500 \mathrm{~ms}$

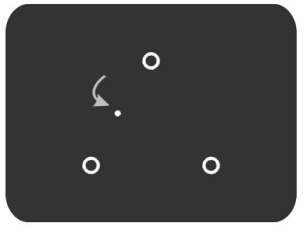

Reproduction of target location with mouse cursor

Fig. 35a: Landmarks and possible target locations in the four conditions of Experiment 9. b: Time course of a trial.

\section{Stimuli.}

The apparatus was as described in Experiment 6. The stimuli were identical to those used in the previous two experiments but appeared in new arrangements. All displays consisted of configurations of three landmarks. In one quarter of the trials, landmarks were arranged in an equilateral triangle with a side length of $2.89^{\circ}$, always in upright $\left(0^{\circ}\right)$ orientation, with 18 possible target locations. In the remaining trials, equiscleral configurations were generated by stretching the triangles along one of the three lines of symmetry while leaving the two remaining landmarks at their location (Fig. 35a). Resulting configurations had a narrow angle of $40^{\circ}$; their axis of elonga- 
tion had an orientation of either $0^{\circ}, 120^{\circ}$, or $240^{\circ}$ (counterclockwise). The grid of possible target locations was stretched accordingly. A dynamical mask was used as described in Experiment 1.

\section{Procedure.}

A trial began with the appearance of the target together with the three landmarks for $750 \mathrm{~ms}$. The target was then replaced by the dynamic mask for $500 \mathrm{~ms}$ while the landmarks remained visible. Immediately after the masking interval, a mouse cursor looking exactly like the target appeared randomly in one of eight possible locations beyond the visible edges of the monitor (see Experiment 6 for details). After the participant had reproduced the target position, a $1000 \mathrm{~Hz}, 100 \mathrm{~ms}$ tone was sounded for feedback that the response had been registered. After an intertrial interval of $500 \mathrm{~ms}$, a new trial began. Participants were instructed to work quickly, but it was emphasized that accuracy rather than speed was important.

Stimulus conditions were counterbalanced such that each combination of landmark configuration (equiscleral $0^{\circ}, 120^{\circ}, 240^{\circ}$, and equilateral), and target position occured quasi-randomly and equiprobably, with each combination appearing once every three blocks. The starting position of the mouse cursor was randomly determined independently in each trial. After each block, participants received summary feedback about their average euclidean deviation from the true target position, rounded to the nearest pixel. Participants performed one session of 30 blocks with 24 trials each. Each session started with an additional practice block of 24 trials with stimuli drawn randomly from the experimental blocks. Practice trials were not analyzed. After the final session, participants were debriefed and received an explanation of the purpose of the experiment.

\section{Data treatment.}

Criteria for outlier elimination were as described in Experiment 7. The procedure eliminated $1.93 \%$ of the raw data.

\subsubsection{Results}

The vector fields from the equilateral condition were analyzed using MANOVA with the horizontal and vertical components of distortion as dependent variables and 
target position as independent variable. Participants were included as a random factor.
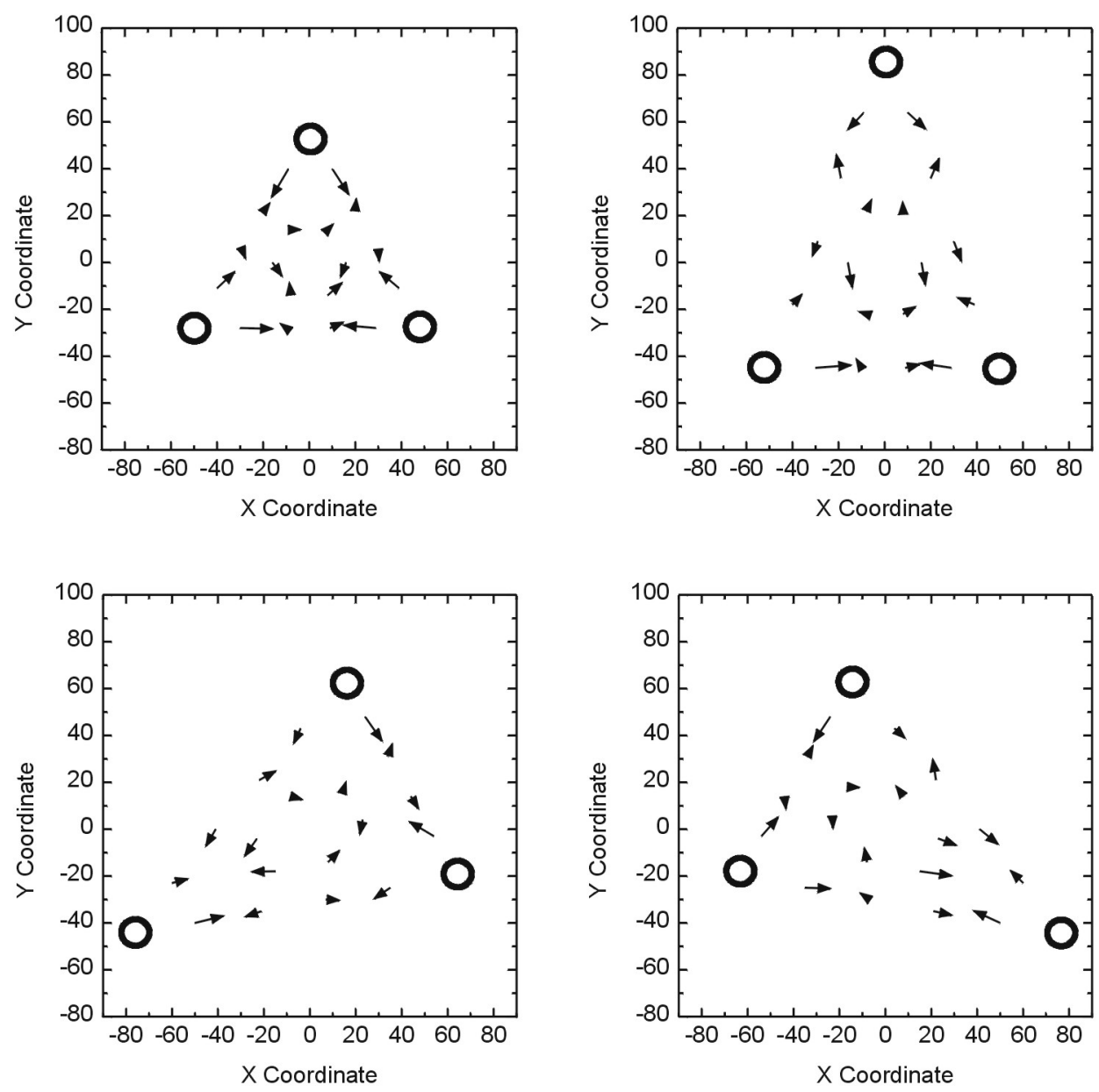

Fig. 36: Distortional fields in Experiment 9. Arrows are magnified by a factor of 2.5 for readability.

\section{Equilateral condition.}

In the equilateral condition, there was striking symmetry of spatial distortions about all three possible axes (Fig. 36). In line with the results from Experiments 7 and 8, reproductions were biased away from the landmarks and also slightly away from the midpoints of the triangle's sides, with these vectors hardly departing from the virtual lines comprising the triangle. MANOVA confirmed that vectors of distortion depended on the location of the target, $\Lambda(34,304)=0.357, p<.001$, this effect being significant for both the horizontal and vertical components, $F(17,153)=5.961$ and 6.122, MSE $=91.304$ and 69.658, respectively, both $p<.001$. Correlational analyses 
of symmetry (Fig. 31) showed that symmetry was significant about all possible axes, all $r_{x x^{\prime}} \leq-.813$, all $r_{y y^{\prime}} \geq .820$, all $p<.005$. Symmetry about the vertical axis was better than symmetry about the $240^{\circ}$ axis, $z=3.235, p<.001$ for the vertical component, $z$ $=0.024, p>.50$ for the horizontal component. It was also marginally better than symmetry about the $120^{\circ}$ axis, $z=1.554, p=.060$ for the vertical component, $z=-$ 1.640, $p=0.505$ for the horizontal component. The oblique axes differed slightly from each other, $z=1.681, p<.05$ for the vertical component, $z=1.663, p>.90$ for the horizontal component. Computation of the symmetry index described in Experiment 7 revealed that symmetry residuals were about five times larger for the oblique than for the vertical axes (Fig. 32).

\section{Equiscleral conditions.}

The three remaining conditions with equiscleral triangles were subjected to MANOVA after appropriate normalisation by rotating the axis of elongation into a vertical position. MANOVA confirmed that the vectors of distortion depended on target position, $\Lambda(34,304)=0.342, p<.001$. This effect was significant for the $x$ as well as for the $y$ components, $F(17,153)=6.290$ and $6.489, M S E=163.390$ and 228.590 , respectively, both $p<.001$. There was no main effect of orientation, $\Lambda(4,34)=0.811$, $p>.40$, but a significant interaction of both factors indicating that vector fields differed across orientations, $\Lambda(68,610)=0.683, p<.001$, which was significant for both the horizontal and vertical components, $F(34,306)=1.789$ and $2.005, M S E=39.745$ and 44.375, respectively, both $p<.01$.

Analyses of symmetry proceeded by transforming the vector fields in such a way that three axes of symmetry could be evaluated. After normalising the vector fields so that the axis of elongation was vertical, they were compressed along the vertical axes until they were equilateral again (from here on, this transformation will be referred to as the "congruence transformation"). Analysis then proceeded as described in Experiment 7.

For the vector field in $0^{\circ}$ orientation with its axis of elongation coinciding with the allocentric vertical, correlational analyses shown in Fig. 31 revealed significant symmetry about the $0^{\circ}$ axis $\left(r_{x x^{\prime}}=-.956, r_{y y^{\prime}}=.972\right.$, both $\left.p<.001\right)$, but weaker symmetry about the $120^{\circ}$ axis $\left(r_{x x^{\prime}}=-.479, p>.05, r_{y y^{\prime}}=.816, p<.005\right)$ and the $240^{\circ}$ axis $\left(r_{x x^{\prime}}=-.585, r_{y y^{\prime}}=.616\right.$, both $\left.p<.05\right)$. Symmetry was better about the axis of elongation than about the $120^{\circ}$ axis, $z=-3.639, p<.001$ for the horizontal component, $z=$ 
$2.600, p<.005$ for the vertical component. It was also better than about the $240^{\circ}$ axis, $z=-3.247, p<.001$ for the horizontal component, $z=3.727, p<.001$ for the vertical component. The oblique axes did not differ in symmetry, both $p>.10$.

For those vector fields with their axes of elongation departing from allocentric vertical, symmetry differences across the three axes were less pronounced. In both orientations, there was significant symmetry about all possible axes, all $r_{x x^{\prime}} \leq-.665$, all $r_{y y^{\prime}} \geq .688$, all $p<.05$. For the triangle in $120^{\circ}$ orientation, symmetry was better about the axis of elongation than about the axis running through the upper landmark, $z=-$ 2.500, $p<.001$ for the horizontal component, $z=0.319, p>.35$ for the vertical component, but not different from the axis running through the lower right landmark, both $p>$.15. Also, these two axes did not differ significantly, both $p>.25$. For the triangle in $240^{\circ}$ orientation, symmetry was somewhat better about the axis of elongation than about the axis running through the upper landmark, $z=1.730, p<.05$ for the vertical component, $z=-0.443, p>.30$ for the horizontal component, but not different from the axis running through the lower left landmark, both $p>.10$. Again, these two axes did not differ, both $p>.05$.

Results from the symmetry index agreed with the correlational approach but revealed interesting quantitative relationships among the conditions (Fig. 32). In the equilateral configuration, the vertical axis of symmetry had much smaller symmetry residuals than the two oblique axes. These residuals were about the same size as those observed for the axes of elongation in the equiscleral conditions, all indices being of similar size. Thus, after appropriate congruence transformations, the amount of symmetry about the vertical axes in the equilateral condition was the same when the triangle was oblonged along that axis, and even when this oblonged figure's orientation was changed. Second, in oblonged triangles, the axis of elongation displayed stronger symmetry than the other axes after a congruence transformation, regardless of stimulus orientation.

Third and most surprising, symmetry about the oblique axes in equiscleral triangles was not worse (and even slightly better) than symmetry about the corresponding axes in the equilateral condition. This is striking because symmetry is only implicit here and can be derived only after the congruence transformation has taken place. It should be noted that this is not an artifact of the congruence transformation, because the results for the vertically oblonged triangle are consistent with those in the control condition. 


\subsubsection{Discussion}

Using elongated equiscleral triangles does not qualitatively change the pattern of distortions observed in visual short-term memory. Distortions take place along the virtual sides of the triangles, with distortion away from the landmarks and away from the midpoints of the triangles' sides. Patterns of distortion are strikingly symmetric and seem invariant with respect to the orientation of the landmark configuration, obviously taking place in a stimulus-centered system of reference.

However, the data also clearly establish an interaction of intrinsic and extrinsic systems of reference in spatial memory distortions. First, as observed in Experiment 7 , the allocentric vertical is the best axis of symmetry in an equilateral triangle, suggesting that alignment with an extrinsic reference system enhances symmetry. In contrast, for elongated triangles, the amount of symmetry about the axis of elongation does not depend at all on its alignment with the allocentric vertical. It seems that the axis of elongation is able to impose such a strong reference system that all additional effects of alignment are overruled. There might also be a floor effect at work here; because the symmetry index is a euclidean measure, it is sensitive to both the mean and variance of vector differences, reaching zero only if the variance is zero. Because some variable error in reproductions is indispensable, weak effects of alignment might be too small to reduce the index even further.

Inspection of Fig. 36 suggests that distortional fields for elongated triangles of landmarks are only a stretched version of the equilateral condition. Results from the symmetry index give some striking corroboration for this idea because they show that implicit symmetry properties can be retrieved if a congruence transformation is performed such that symmetry about all three axes can be assessed again. When this is done, symmetry along all three lines is just as strong or even stronger than that observed in a configuration of triangles that was equilateral from the beginning. This suggests some kind of "rubber-sheet geometry" where new distortional fields can be predicted by just performing the geometrical transformation appropriate for the intended configuration of landmarks. This should be possible at least for simple transformations like rescaling and stretching the landmark configuration, provided that intrinsic stimulus properties impose a strong enough system of reference. 
Together, Experiments 7 to 9 clearly show that alignment or misalignment with extrinsic reference systems plays a role for determining the geometric properties of spatial memory distortions, most notably their symmetry properties. This finding is inconsistent with all theoretical approaches assuming that distortional fields are determined in a strictly bottom-up, stimulus-driven manner, like the ones by Nelson and Chaiklin (1980) and Watson (1977). Although Huttenlocher et al.'s (1991) model principally allows for strategic effects, it depends on the observer's setting up a system of spatial categories in short-term memory. It is at least not obvious which kind of category system would lead to the observed effects, and it seems impossible to determine this reference system from the observed pattern of reproductions. 


\section{General Discussion}

The experiments reported in this thesis were conducted to yield insight into some basic issues of the spatial organisation of visual short-term memory. In this final section, theoretical implications of the data will be discussed with respect to the following problems: how landmarks reduce spatial uncertainty associated with target position, how they spatially distort visual memory, how the effects of multiple landmarks combine, and which spatial reference systems are at work. It is argued that none of the two major models of spatial memory distortions can account for the whole range of effects, and that both models include important assumptions that are proved wrong by some aspects of the data. Finally, a tentative neural network model of spatial memory distortions (Trommershäuser, 2001) is shown to be able to explain most of the phenomena here by assuming that spatial distortions take place by attentional preactivation in topographic maps stored in prefrontal cortex.

\subsection{Reduction of spatial uncertainty and the origin of spatial distortions}

All experiments reported here consistently show that landmarks alter the spatial structure of short-term memory representations. In particular, landmarks affect both the variable and systematic error of targets reproduced after some short retention interval. In this section, I will try to systematize these effects and show that variances and spatial biases are crucial components of landmark effects that must be discussed in conjunction.

In the vicinity of a visual landmark, the variance of reproduced target locations is markedly reduced. This effect is well illustrated in the scatterplots from Experiment 2 comparing one-landmark and no-landmark displays, where it can be seen that the landmark not only reduces variable error but also serves as a spatial anchor that prevents targets from drifting around in spatial memory. The reduced variability in target reproductions is accompanied, however, by systematic spatial biases. These biases are also clearly resulting from the presence of visual landmarks, since their pattern is strongly dependent on stimulus geometry. In particular, the pattern of biases follows several types of geometrical transformations in the layout of landmarks, like rotation (Experiments 3, 6, and 7 - 9), expansion/compression, and translation (Diedrichsen, 1998). Experiment 9 suggests that even stretching an equilateral triangular configu- 
ration of landmarks to form an elongated triangle simply leads to a stretching of the distortional fields, as if occuring in some kind of "rubber-sheet geometry" implicitly preserving symmetry about all three axes of the previously equilateral configuration.

The simple patterns of distortion observed for single landmarks seem to generalize quite naturally to more complex configurations of two or three landmarks. Presenting a landmark in the form of a large circle leads to an outward distortion away from the center of the circle as well as from the circular line itself (Experiment 1), although this effect is not consistent across participants. When a single dot is presented as a landmark (Experiment 2), there is always some tendency to reproduce the target too far away from the landmark, but this effect is much larger near the landmark. Without any landmark, however, distortion might be even larger albeit less systematic, due to the fact that the whole distribution of target representations might drift in visual memory. At the same time, euclidean error can be smaller near the landmark because of the reduction in variance. Advancing from a one-landmark to a two-landmark configuration leads to distortion away from the midpoint in addition to distortion away from the landmarks themselves (Experiments. $3-6$ ). This pattern is largely invariant regarding the orientation of the two-landmark configuration, except for a global downward bias in the lower part of the visual field which becoming especially important when extrinsic spatial reference cues are eliminated (Experiment 6). Finally, when three landmarks are presented, distortion is away from the center of gravity of the configuration, from the center of the virtual lines connecting the landmarks, and from the landmarks themselves (Experiments $7-9$ ).

In general, every time a new landmark is introduced, predictable patterns of distortion seem to be generated, with biases directed away from the center of gravity, from the landmarks themselves, from the midpoints of virtual lines connecting the landmarks, and from the virtual lines themselves. Further research is needed to confirm this hypothesis with more complex and more general landmark configurations, which might lead to a small set of simple rules able to predict distortional fields of arbitrary configurations.

Several authors have hypothesized that a reduction of spatial variance near visual landmarks inevitably leads to introduction of spatial bias. For example, Huttenlocher, Hedges and Duncan (1991) propose that the distribution of target positions in visual memory is restricted not to overlap conspicuous spatial boundaries, leading to truncation of those parts of the distribution that overlap the boundary, and this in 
turn leading to a skewed distribution with expectancy shifted away from the boundary. Thus, the closer the distribution is to the boundary, the larger the part truncated, the reduction in variance, and the amount of spatial bias generated. Taking a different approach, Suzuki and Cavanagh (1997) propose that spatial reference stimuli gather attentional resources, which might affect receptive field properties of cells encoding the space near the stimuli. In particular, receptive fields might shift towards the attended stimulus, might narrow their tuning curves in the vicinity of that stimulus, or inhibit cells with receptive fields farther away from the stimulus. All these mechanisms would lead to a reduction in variable error but would also induce a systematic shift in target representation away from the focus of attention. I will discuss the role of attention in more detail in the final part of this discussion. The results presented here are consistent with Suzuki and Cavanagh's explanation but not the Huttenlocher et al. model. This model predicts that spatial representations are biased away from category boundaries because of the uncertainty of category membership for targets located near the boundary, which would imply high locational uncertainty and large variable error for the midpoint between two landmarks. In contrast, all datasets reported here consistently show that this region has markedly reduced variable error, which provides direct evidence against Huttenlocher et al.'s (1991) theory.

Results are also at odds with Nelson and Chaiklin's (1980) weighted distortion theory. Their model assumes that distortions are directed towards landmark stimuli (in their experiments, points comprising a circle or ellipse), not away from them. They further assume that the distortion is stronger for locations farther away from the landmark, leading to the erroneous prediction that bias should be small near the landmark and become large only at some distance from it. This is probably based on a misinterpretation of the distortions in their circular displays: distortion is not towards the circumference but rather away from the midpoint of the circle, which is easily explained by assuming that participants use the midpoint strategically as a spatial reference. More importantly, the nonmonotonous spatial course of distortions around single landmarks (Experiments 5 and 6 ) provides strong evidence against this model. Interestingly, this also means that spatial memory distortions cannot simply be inferred from the standard psychophysical function for remembered distance which was the prime source of Nelson and Chaiklin's assumptions. Modelled as a power function with exponent less than 1 (Gescheider, 1997), such a function would make 
predictions similar to Nelson and Chaiklin's model but could not account for the local distortion away from the landmark.

Diedrichsen and Werner (submitted) have shown that distortional effects develop over time, being detectable after a retention interval of only $50 \mathrm{~ms}$ and becoming asymptotic within $400 \mathrm{~ms}$. However, Experiment 4 suggests that distortional effects might reach an asymptote as early as $100 \mathrm{~ms}$ after target offset, thus showing a much more rapid time-course. Recent data from our laboratory also show that the distortions arise mainly during the first $100 \mathrm{~ms}$, with some additional distortion between 100 and 200 ms, which also suggests a somewhat faster build-up of distortions than estimated by Diedrichsen and Werner. One reason for this discrepancy might lie in the different psychophysical techniques used. In the task employed by Diedrichsen and Werner, the target was shifted during the masking interval in a majority of trials, and participants had to detect whether or not the translation had occured. In Experiment 4 reported here, they had to discriminate the direction of the translation. It is known from signal detection theory (Macmillan \& Creelman, 1991) that detection tasks are often subject to response biases that are difficult to assess in Diedrichsen and Werner's experiment and that might distort the estimates. In comparison, response biases in discrimination tasks are usually not confounded with sensitivity effects because they tend to cancel out as long as participants choose each response alternative about equally often. Therefore, the discrimination paradigm of Experiment 6 might provide the more accurate estimate of the time-course of spatial memory distortions.

Whatever the exact time-course of the effect might be, it is clear that distortions are dynamical and gradually build up over time. This implies that the distortions measured here do not just comprise a bias present during the decision where to place the reproduced target or perhaps even during the corresponding motor response, but that the spatial representation itself is subject to gradual change. This is not consistent with Nelson and Chaiklin's (1980) model unless it is assumed that psychophysical functions of remembered target-to-landmark distance change their shape during the masking interval (but note that even then, the model would not be able to account for distortional effects near the landmarks). In contrast, Huttenlocher et al.'s (1991) model can account for dynamical effects quite naturally if it is assumed that representations become increasingly noisy during the retention interval, which would lead to stronger truncation as well as prototype effects (however, remember 
that the actual pattern of variances across the visual field is grossly mispredicted by the model). Finally, a model based on attentional effects within a topographical neuronal map could account for the observed effects in a neurobiologically plausible way without invoking higher-level cognitive functions. Discussion of such a model is deferred until the final section of this discussion.

\subsection{Nonlinear combination of local distortional fields}

Although patterns of spatial memory distortions often remind observers of physical force fields, their formal properties are entirely different. In stark contrast to the fields encountered in gravity or electromagnetism, distortional fields of single landmarks do not combine linearly. Instead, single landmarks combine by partitioning the visual field so that the compound pattern of distortion is determined by the local patterns of distortion around each landmark.

This is shown in Experiments 5 and 6. When single landmarks are presented either in the left or right halves of the visual field, it is not possible to form a linear combination of their distortional fields that can account for the joint effect resulting when both landmarks are presented simultaneously. For example, simply superimposing single-landmark fields leads to poor predictions of the actual data. Among other things, such a model wrongly predicts horizontal distortions directly above or below the landmarks, fails to account for distortions away from the midpoint between the landmarks, and overestimates the amount of distortion in many regions of the display. Variance predictions follow from it only under the assumption that landmarks are the only sources of variance in the system, an assumption that Experiments 1 and 2 show to be wrong. Even with this assumption granted, successive superposition of single-landmark fields would lead to an accumulation of variable error which is incompatible with the variance-reducing effects of visual landmarks demonstrated in Experiments 1 and 2 .

While simple superposition fails to account for the data, allowing the singlelandmark patterns to add linearly but with free weighting parameters yields a potentially more powerful model. However, even this model fits the data very poorly because it tends to produce some averaging of the single-landmark fields, which leads to severe underestimation of spatial biases in all regions of the display. Variable error (subject to the same assumptions as in the superposition model) is underestimated 
even more, since variances add with the squares of their weights. Both linear models therefore yield a very unsatisfactory fit to the data.

Surprisingly, a very simple partition model without any free parameters accounts very nicely for the patterns of both systematic and variable error. This is possible because the distortional fields near the landmarks are almost identical in the one-landmark and two-landmark displays: the local distortional field around a landmark is the same regardless of whether a second landmark is placed somewhere else in the field. In contrast to the linear models, the partition model very straightforwardly allows for variance predictions, preserving local variance patterns around single landmarks because it doesn't use any information from the field half opposite to the landmark.

Experiment 1 also suggests that the influence of a landmark is spatially restricted. Its pattern of euclidean error (Fig. 4) clearly shows that error is small near the landmark but quickly increases with distance. Importantly, however, the amount of error finally reaches the same value that would have been observed in a control condition without a landmark near the target, implying tight spatial restrictions of the variance-reducing effect of the landmark. This finding suggests that landmarks attract locally restricted attentional resources, perhaps similar to the well-established notion of a "spotlight of attention" (Eriksen \& Eriksen, 1974; LaBerge, 1995), where only target positions within the spotlight profit from the deployment of attention.

Whereas the Huttenlocher et al. (1991) model does not make any predictions for combinations of single-landmark fields, the model proposed by Nelson and Chaiklin (1980) assumes that such fields superimpose, but with a nonlinear weighting function decreasing with distance from the landmark. Of course, this is correct in principle, because the step function used in the partition model is an extreme example of such a weighting function. However, the Nelson-Chaiklin model fails to recognize that landmarks are partitioning visual space, a property that is only true of weighting functions having a steep increase exactly at the midpoint between the landmarks. In their model, the weighting functions do not depend on stimulus geometry at all.

The partition principle leads quite naturally to predictions valid for natural visual scenes we encounter in everyday life. Most stimuli that fill our visual fields are crowded with spatial cues, all of which might induce a local pattern of spatial distortions. According to the partition model, all these local effects should mutually restrict each other, forming a fine-grained partition of the field where distortions can never 
become very large - indeed, the distortions observed with three-landmark displays are substantially smaller than those observed with two-landmark displays. In contrast, linear models would predict all these distortional effects to spread throughout the entire display. In the case of a superposition model, this would likely lead to an accumulation of variable error.

The partition principle might therefore play an important role in other problems of visual perception. For example, partition of a complex configuration of landmarks might help reveal the intrinsic structure of the visual object, like axes of symmetry and elongation. In Experiments 7 to 9, the partition principle leads to partition boundaries that run along the symmetry axes of triangular landmark configurations. In Experiment 9 , the partition boundary might help retain "implicit symmetry", the ability to maintain symmetry about a line when a triangle is stretched out of shape. Note that if a formerly equilateral triangle is elongated, symmetry about all but one of the possible axes becomes undefined whereas partition boundaries do not. The partition principle might thus help to establish organizational and grouping principles (Wertheimer, 1923). Recently, Palmer and Rock (1994) have suggested that one of the first steps in extracting information form a two-dimensional image is partitioning it into segregated regions following a principle of uniform connectedness, which states that contiguous regions of uniform stimulus features will be perceived as unitary objects.

While distortions at the landmarks are readily explained by attentional models, distortions from the midpoint between two landmarks can only be explained under the assumption that the midpoint is strategically used as an additional spatial reference a "virtual landmark". Thus, the midpoint has a similar status as other "virtual" lines of symmetry and partition. Bisection points have been shown to play an important role in psychophysical tasks involving judgment of proportions (Hollands \& Dyre, 2000). Such virtual lines might be similar to illusory contours, which have been shown to drive about $40 \%$ of cells in V2 in much the same way that real contours do (von der Heydt \& Peterhans, 1989). It is possible that some cells in dIPFC are similarly unable to distinguish real lines from virtual constructions. Again, further discussion of such virtual representations in the context of a computational model is postponed until the final part of this discussion. 


\subsection{Interactions of intrinsic and extrinsic reference systems}

It is clear from most experiments reported in this thesis that the pattern of spatial memory distortions closely follows the geometrical properties of the landmark configuration. Landmarks obviously form an intrinsic reference system with properties depending strictly on the spatial layout of the landmarks.

If intrinsic reference systems were the only reference systems determining the distortional field, distortions would exclusively depend on the geometrical properties of the landmark configuration. Indeed, there is a remarkable range of geometrical transformations that can be performed on a landmark configuration with distortional patterns closely following, including translation, rotation, scaling, and stretching. However, some qualifications are also necessary.

First, the pattern of distortions is not always strictly regular. Especially when spatial cues from the laboratory environment are eliminated, some distortional effects become apparent that seem not at all dependent on the landmark configuration but instead homogenously affect the entire visual field. Most notably, in Experiments 1 and 6 there is a marked overall tendency to report targets too far below the point where they were actually presented. This downward bias is especially pronounced in the lower half of the visual field and seems to disappear when some spatial reference is placed there, like the lower edge of the monitor in Experiment 5, or the lower landmark in the vertical configuration of Experiment 6 . These findings suggest a role for spatial distortions that are organized within an extrinsic frame of reference, for example gravity (Hubbard, 1995; Finkelmeyer, 2000).

Second, Experiments 7 to 9 examine symmetry properties of distortional fields when axes of symmetry are aligned or misaligned with extrinsic axes. These experiments show that symmetry about a particular axis is stronger when that axis is aligned with the allocentric vertical; however, when the symmetry axis is also the axis of elongation, additional alignment with the allocentric vertical does not enhance symmetry any further. One might therefore conclude that extrinsic reference systems markedly alter the pattern of spatial memory distortions only if some important boundary conditions are met. These conditions include displays where all except a very few spatial reference cues are eliminated or where cardinal axes of a landmark configuration are ambiguous, as they are for equilateral configurations. As soon as configurations have clearly defined cardinal axes, their intrinsic geometrical properties seem able to overrule extrinsic biases. 


\subsection{Selective attention}

None of the two major models of spatial memory distortions gives a fully satisfactory account of the experimental data. In particular, they both fail to explain some apparent inconsistencies in the data that strongly hint at an attentional explanation.

One problem is that distortional fields may markedly vary between participants even under identical stimulus conditions. Although most participants show a repulsion effect from landmark stimuli, only some of them do also show distortion away from the midpoint (e.g, there was no evidence for such an effect in the horizontal condition of Experiment 6). Moreover, repulsion from the midpoint seems totally absent when participants are pointing with a stylus to reproduce target location (Diedrichsen, 1998). One tempting explanation for the latter effect would be that reproduction with the mouse cursor and reproduction by pointing are controlled by different spatial maps, perhaps belonging to a ventral, "cognitive" system and a dorsal, "visuomotor" system, respectively (Milner \& Goodale, 1995; Bridgeman, Peery, \& Anand, 1997). However, this account would only beg the question how these different maps could have so different properties. For example, if spatial distortions stem from lateral interactions of adjacent receptive fields, it is difficult to conceive of two maps with identical properties in some parts of the visual field but contrary properties in another part (namely, around the midpoint).

Instead, the seemingly opposing results can be reconciled with strategic effects within an attentional framework. In particular, participants might use different encoding strategies to memorize a target point with respect to different parts of the landmark configuration, which might lead to different patterns of distortion near the midpoint. Direct evidence for such processes comes from a psychophysical model by Hollands \& Dyre (2000) concerning the estimation of proportions, e.g., in line bisection. In their model, they assume that proportions along a psychophysical continuum are strategically coded with respect to virtual anchoring points that might differ between observers. This results in a sectioning of the continuum into parts, each of which is distorted according to a standard psychophysical function for length estimation. In this way, a cyclical pattern of misestimations occurs, with the number of cycles depending on the number of anchoring points an observer will use. The authors show that their model gives an excellent fit to the data reported by Huttenlocher, Hedges, \& Duncan (1991). Thus, strategical factors can explain the somewhat vari- 
able effect of distortions around the midpoint, which might act like a "virtual landmark" in some participants but not in others. Such strategic factors might also account for the nonhomogenous properties of symmetry axes in equilateral triangles, which could be readily explained by assuming that participants attentionally select the vertical or near-vertical axes as a cardinal axis of the triangle.

Yet, this does not explain why there is no repulsion from the landmarks when the target location is reproduced by pointing with a stylus (Diedrichsen, 1998). However, one crucial difference between the pointing and mouse cursor conditions in Diedrichsen's experiments is that the initial position of the mouse cursor was unpredictable, whereas the pointing responses always had to start from the same point in the lower middle part of the display. This might cause attention to be distributed differently in the two conditions: specifically, participants might encode the target with respect to the starting point of the movement. Although this argument might seem far-fetched, recent data from our laboratory show that the predictability of effector locations is crucial (Trommershäuser, 2001; Trommershäuser \& Schmidt, in prep.). In one of these experiments, we varied the predictability of the initial mouse cursor position: in one condition, the cursor always started from the midpoint between the landmarks; in the other condition, it started unpredictably from one of eight locations arranged circularly around the display. In either condition, the cursor was not visible unless moved by a few millimeters. With unpredictable starting positions, we found a distortional pattern similar to those in Experiments 3, 5, and 6, with repulsion from the landmarks as well as from the midpoint, thus replicating Diedrichsen's mouse cursor results. In contrast, with the cursor starting predictably from the midpoint, we found repulsion only from the midpoint but not from the landmarks, thus replicating Diedrichsen's pointing results. It seems that the cursor's starting position created a point of very high positional certainty, inviting strategies of encoding the target relative to this point rather than relative to the landmarks.

In a subsequent experiment, we could produce the same dissociation by manipulating whether participants attended or ignored the landmarks, leaving both visual stimuli and motor responses identical. Landmarks were not visible during the masking interval but reappeared either at the same position as before (in $50 \%$ of the trials) or at a displaced position. Participants had to reproduce the target either rela

\footnotetext{
2 Thanks to Jochen Müsseler for making the important suggestion that the cursor's starting position might have an influence.
} 
tive to the landmarks (thus forced to attend to the landmarks) or with respect to the absolute screen position (thus trying to ignore the landmarks). The mouse cursor always started randomly from outside the display. We compared only those trials where landmarks were not displaced, which were identical with respect to stimuli and responses and differed only in attentional set. When the target was reproduced with respect to the landmarks, we found repulsion from the landmarks as well as from the midpoint, again reproducing Diedrichsen's mouse cursor results. In contrast, when landmarks could not be used as a spatial reference at encoding, we found repulsion only from the midpoint but not from the landmarks, thus reproducing Diedrichsen's pointing results.

In sum, whether landmarks act as repulsors or not seems to depend strongly on whether they are attentionally selected as a spatial reference. If this is the case, attentional mechanisms like those described by Suzuki and Cavanagh (1997) might bias the target representation away from the focus of attention centered at the landmark. When the landmarks cannot be used as a spatial reference, other points of high positional certainty will attract attention, such as the starting position of a pointing movement or the center of the screen.

\subsection{An attentional preactivation model of spatial memory distortions}

In this final section, I will advance an attentional theory of distortional effects that can explain many of the phenomena we have observed so far. Based on earlier neural network models of prefrontal cortex (e.g., Camperi \& Wang, 1998), this attentional account was developed in our laboratory by Julia Trommershäuser (2001).

Her model assumes that when landmarks and targets are presented at the beginning of a trial, a visual representation of these stimuli is formed that will have reached working memory by the beginning of the masking interval. Because successive transformations of this representation proceed through neuronal maps with everincreasing visual fields (i.e., from early visual high-resolution areas to low-resolution population-coded maps in the frontal lobe), random noise is added to the signal, introducing some spatial uncertainty. Finally, however, visual stimuli are represented in topographical fashion in dorsolateral prefrontal cortex. During the course of a typical trial, the memory representation of the landmarks will continue to receive visual input whereas input from the target is disrupted at the beginning of the masking interval. 
The crucial assumption of the model is that neuronal maps in visual short-term memory are structured by attentional preactivation of certain points and lines. The pattern of preactivation defines the spatial reference system currently active in memory and depends mainly on the visual stimulation. Note that this is in line with Psotka's (1978) results showing that participants might preferentially select virtual lines when asked to arbitrarily place a single dot into a surrounding shape, giving some independent validation to our concept of attentional preactivation. Note also that Psotka's patterns are generally consistent with the distortional fields observed in our stimulus configurations. For a two-landmark display, attentional preactivation might involve the landmarks, the connecting line, the midpoint, and the perpendicular line through the midpoint. Thus, spatial reference systems are assumed to be represented in spatial memory in much the same way that illusory contours are represented in early visual representations. We do not assume that all these lines are being attended all of the time, which would imply very demanding attention-splitting abilities of the memory system. Rather, it suffices to assume that attention will visit virtual lines with higher probability than other regions.

In Trommershäuser's model, the pattern of preactivation is jointly determined by intrinsic reference systems (stimulus centered), extrinsic reference systems (possibly independent of stimulus properties), and strategical employment of attention. During the masking interval, the memory trace of the target is superimposed on the pattern of preactivation, which continues to receive visual input from the still visible landmarks. Target location is retrieved from the map by localizing the maximum increase in activation beyond preactivation, i.e., the point where the difference distribution of the final and preactivation patterns is maximal.

If the visual signal and the pattern of preactivation were strictly additive, the difference distribution would be spatially unbiased, and the target could be reproduced veridically, albeit with some spatial uncertainty. However, the model assumes that the firing rates are not strictly additive but subject to saturation because there is an upper limit to the overall firing rate. If preactivation is already high, additional activation from the target stimulus is very restricted, whereas with low preactivation the additional activation has a larger impact (Byrne, 1999). It turns out that with neural saturation, the difference distribution is skewed away from preactivated regions.

The model is implemented in an artificial neuronal network with an input layer and a memory layer. For simplicity, the input layer is modelled as a sheet of inde- 
pendent neurons that are directly activated by the visual stimuli, e.g. constituting a veridical map of two landmarks. Each neuron in the input layer is connected to a range of neurons in the memory layer, with the weights of these connections following a Gaussian distribution decreasing with distance from the actual target location. 3 The input layer therefore simply projects a fuzzy image of the stimuli onto the memory layer, which is added to the memory layer's preactivation pattern according to a saturation function. A target location is retrieved from the memory layer by finding the neuron with maximum increase in activity exceeding the preactivation level. The model makes use of five free parameters, four less than Huttenlocher et al.'s (1991) prototype model.

Trommershäuser (2001) found that the model is able to predict the pattern of distortions obtained in the two-landmark displays very well, correctly accounting for spatial memory distortions away from the landmarks and from the midpoint between the landmarks. The amount of distortion depends on the amount of attention payed to the landmarks: with higher preactivation of a landmark, the spatial bias around it becomes stronger, which was confirmed in an additional experiment. Importantly, the model also accounts for the pattern of variable error, predicting markedly reduced variances at preactivated locations, including landmarks and midpoint.

The model can also explain other aspects of the data. For example, it predicts that distortion will increase with mask duration when spatial information in the memory layer decays or dissipates, leading to more noise in the representation, broader distributions of target and preactivated regions, and therefore stronger skewing of the target's difference distribution. Of course, it also predicts that distortional fields will closely follow intrinsic frames of reference. Because the distortional effects are strictly local, the model predicts that the combined fields of single landmarks will be locally additive, i.e., form a partition of the visual field.

Currently, the model still has some shortcomings. First, it is not able to account for the complex patterns of spatial distortions obtained with single landmarks (see, e.g., Experiments 3 and 4). Second, it is not yet clear how the subtle interactions of extrinsic and intrinsic reference systems observed in Experiments 7 to

\footnotetext{
3 The network was actually trained to acquire this pattern of connections from an initial random pattern. A Kohonen algorithm (von der Malsburg, 1974; Kohonen, 1982; see Rojas,1993, for an introduction) was used that allowed the network to find the optimal weights by self-organization based only on interactions between neighbouring cells. Kohonen-type networks are generally regarded as biologically plausible models of neural map formation.
} 
9 might be built into the model except by using elaborate ad hoc patterns of preactivation.

\subsection{Conclusions}

The use of simple stimuli in investigating spatial distortions in short-term visual memory yields interesting insights into the mechanisms of spatial memory. It is proposed here that visual landmarks serve to reduce spatial uncertainty in topographical neural maps by possible local mechanisms of receptive field recruitment, tuning, or lateral inhibition (Suzuki \& Cavanagh, 1997) or by preactivation of memory maps probably held in dorsolateral prefrontal cortex (Trommershäuser, 2001). These processes are highly dynamic, are reaching an asymptote within 100 to $200 \mathrm{~ms}$, and are reliably predicted from the pattern of visual stimulation.

The current models of spatial memory distortions are both falsified by the data reported here. Nelson and Chaiklin's (1980) model explains distortions by the properties of psychophysical functions nonlinearly weighted with landmark distance, an account that fails to account for the defining feature of distortional fields, namely, repulsion from landmarks. Huttenlocher et al.'s (1991) explanation invokes the segmentation of the visual display into spatial categories, which is inconsistent with the pattern of variable error indicating that category boundaries are locations of high instead of low spatial certainty. In contrast, the major findings can be explained in a biologically plausible way by assuming attentional effects within topographical neural maps of visual space.

On the basis of the successful simulations with Trommershäuser's (2001) model, one can make predictions about spatial representations in dorsolateral prefrontal cortex. First, the model correctly predicts that neurons in dIPFC should be able to hold a memory map of the visual targets (Goldman-Rakic, Ó Scalaidhe, \& Chafee, 2000). Second, stimulus-based intrinsic reference systems should show up as a preactivation pattern in prefrontal maps. Third, this preactivation pattern should be subject to attentional modulation and strategy employment. Fourth, preactivation should be high enough to lead to noticable saturation effects in firing rates. We believe it would be worthwhile to evaluate these predictions in single-cell studies.

One might speculate whether spatial memory distortions might also provide some insight into dynamic processes of object segmentation and representation, since distortional fields reveal the system of reference points and lines used to en- 
code an object residing in visual memory. Distortional effects also suggest that spatial representations themselves are highly dynamic, determined by a stable equilibrium of distortions arising from each object in the visual field, but so that all these distortional effects mutually restrict each other by partitioning the visual field. Finally, the dynamic properties of spatial memory distortions might shed some light on processes involved in stimulus localization, for example in mislocalizing a target moving from behind an occluder (Müsseler \& Aschersleben, 1998; Van der Heijden et al., 1999), or in extrapolating the position of a moving stimulus (Nijhawan, 1994; Berry, Brivanlou, Jordan, \& Meister, 1999). Within the present framework, the study of spatial short-term visual memory might be closely linked to visual selective attention in a biologically plausible and biologically testable way. 


\section{Summary}

Two-dimensional topographic maps represent a major coding principle for spatial information in the primate brain. This thesis employs a simple paradigm to yield insight into the properties of those maps by psychophysical methods (Diedrichsen \& Werner, submitted; Schmidt, Werner \& Diedrichsen, submitted). Observers watch a display containing a target stimulus along with one to three small landmark stimuli, which are shown for about $500 \mathrm{~ms}$. The display is then replaced by a 500-ms visual mask that blanks out the target but leaves the landmarks visible. The observer's task is to reproduce the position of the target with respect to the landmarks as accurately as possible, using a mouse cursor.

Experiments 1 and 2 employ single-landmark displays to show that memory representations are systematically distorted by the presence of visual landmark stimuli serving as spatial reference. At the same time, landmarks provide regions of high positional certainty with markedly reduced response variance in their vicinity. Experiment 3 uses displays with two landmarks to show that reproductions are not only distorted away from the landmarks but also from the midpoint between landmarks and from the virtual lines connecting them, forming symmetrical and regular distortional fields. These fields are mainly determined by stimulus-based (intrinsic) reference systems imposed by the spatial layout of the landmarks; they closely follow transformations of these layouts, including translation, rotation and stretching. Experiment 4 uses some of the stimuli from Experiment 3 in a discrimination task where the observer has to judge whether a target reoccuring after the retention interval has been displaced to the left or the right. Because this task allows for very short retention intervals, it can be shown that distortions develop within about 100-200 ms after the original target has disappeared.

Experiments 5 and 6 investigate how the distortional field of two landmarks can be predicted from the distortional fields of the single landmarks presented individually. Targets are presented along with either the left, the right, or both landmarks, with all three types of trial randomly intermixed. Comparison of the single- and bothlandmark fields shows that the pattern of distortions in the display half containing a landmark is the same irrespective of the presence of the other landmark. Thus, landmarks induce a partition of the visual field where each landmark dominates the 
distortional field in one half of the display. Other plausible models of landmark combination, e.g., linear combination or superposition, are clearly discarded.

Finally, Experiments 7 to 9 use triangular configurations of three landmarks having either one or three symmetry axes (equilateral and equiscleral triangles). By rotating the landmark configuration so that one of its symmetry axes coincides with the allocentric vertical as defined by extrinsic reference systems like the body midaxis or gravity, it is shown that distortional fields are not solely governed by intrinsic reference frames. In particular, symmetry about an axis that is aligned with the allocentric vertical is more regular than when the axis is not so aligned. This finding shows that extrinsic spatial reference systems can also influence the symmetry properties of spatial memory distortions, leaving some space for cognitive strategies of some observers' giving certain points and lines special importance as spatial reference stimuli.

The results falsify the two major theories of spatial memory distortions. Nelson and Chaiklin (1980) assume that distortions arise from underestimation of target-tolandmark distance, along with some weighting process that assigns higher relevance to landmarks closer to the target. This model erroneously predicts that memory distortions should increase with distance from the landmarks; it fails to account for the fact that distortions are largest near landmarks or other conspicuous points. A different model by Huttenlocher, Hedges, and Duncan's (1991) assumes that observers segment the display into spatial categories and that distortions arise either when category membership is used to assist in reconstruction of an imprecisely remembered target location or when a target remembered near a category boundary is forcibly assigned to a particular category. This model fails to account for the pattern of variable error: it predicts maximum variance near category boundaries and minimum variance near the center of the category, which is just the opposite of what is observed.

In contrast, a model based on attentional modulations within a topographic neuronal map of visual space (Trommershäuser, 2001) can account for most of the data on spatial memory distortions obtained so far. The model assumes that visual stimuli are represented in a visual memory map, most likely in dorsolateral prefrontal cortex. This map is biased by spatial reference points and lines, which are modelled as patterns of preactivation within the map. Visual activation from the target is supposed to add to the preactivation pattern with some saturation of firing rates, which leads to biases in target representation away from preactivated regions. The model 
successfully accounts for the patterns of distortion as well as response variance; it also correctly predicts that there will be no distortion away from an unattended landmark (Trommershäuser \& Schmidt, in preparation). Successful simuluations with this model lead to predictions of some properties of single cells in dorsolateral prefrontal cortex. 


\section{References}

Aglioti, S., DeSouza, J. F., \& Goodale, M. A. (1995). Size-contrast illusions deceive the eye but not the hand. Current Biology, 5, 679-685.

Agresti, A. (1996). An Introduction to Categorical Data Analysis. New York: Wiley.

Baddeley, A. D. (1990). Human Memory: Theory and Practice. Hillsdale, NJ: Lawrence Erlbaum Associates.

Baird, J. C. (1970). Psychophysical Analysis of Visual Space. New York: Pergamon Press.

Berry, M. J., Brivanlou, I. H., Jordan, T. A., \& Meister, M. (1999). Anticipation of moving stimuli by the retina. Nature, 398, 334-338.

Biederman, I. (1987). Recognition-by-components: A theory of human image understanding. Psychological Review, 94, 115-117.

Blakemore, C., \& Campbell, F. W. (1969). On the existence of neurons in the human visual system selectively responsive to the orientation and size of retinal images. Journal of Physiology, 203, 237-260.

Breitmeyer, B. G. (1984). Visual Masking: An Integrative Approach. Oxford, UK: Oxford University Press.

Bridgeman, B., Peery, S., \& Anand, S. (1997). Interaction of cognitive and sensorimotor maps of visual space. Perception \& Psychophysics, 59, 456-469.

Bridgeman, B., Gemmer, A., Forsman, T., \& Huemer, V. (2000). Processing spatial information in the sensorimotor branch of the visual system. Vision Research, 40, 3539-3552.

Bryant, D. J. \& Subbiah, I. (1993). Strategic and perceptual factors producing tilt contrast in dot localization. Memory \& Cognition, 21, 773-784.

Byrne, J. H. (1999). Postsynaptic potentials and synaptic integration. In M. J. Zigmond, F. E. Bloom, S. C. Landis, J. L. Roberts, \& L. R. Squire (Eds.), Fundamental Neuroscience (pp. 345-362). San Diego, CA: Academic Press.

Camperi, M., \& Wang, X. J. (1998). A model of visuospatial working memory in prefrontal cortex: Recurrent networks and cellular bistability. Journal of Computational Neuroscience, 5, 383-405.

Colby, C. L., Duhamel, J. R., \& Goldberg, M. E. (1995). Oculocentric spatial representation in parietal cortex. Cerebral Cortex, 5, 470-481. 
Connor, C. E., Gallant, A. L., \& Van Essen, D. (1994). Modulation of receptive field profiles in area V4 by shifts in focal attention. Investigative Opthalmology \& Visual Science, 35, 2147.

Crawford, L. E., Huttenlocher, J., Engebretson, P. H. (2000). Category effects on estimates of stimuli: Perception or reconstruction? Psychological Science, 11, 280-284.

Cronbach, L. J., Gleser, G. C., Nanda, H., \& Rajaratnam, N. (1972). The Dependability of Behavioral Measurements: Theory of Generalizability for Scores and Profiles. New York: Wiley.

Desimone, R., \& Duncan, J. (1995). Neural mechanisms of selective visual attention. Annual Review of Neuroscience, 18, 193-222.

DeValois, R. L, \& DeValois, K. K. (1988). Spatial Vision. New York: Oxford University Press.

Diedrichsen, J. (1998). Gibt es ein motorisches Kurzzeitgedächtnis für Positionen im Greifraum? Evidenz aus der Untersuchung kognitiver Verzerrungen. Unpublished Thesis, University of Göttingen.

Diedrichsen, J., \& Werner, S. (2001). The time course of spatial memory distortions. Manuscript submitted for publication.

Downing, C. J. (1988). Expectancy and visual-spatial attention: Effects on stimulus quality. Journal of Experimental Psychology: Human Perception and Performance, $14,188-202$.

Eriksen, B. A., \& Eriksen, C. W. (1974). Effects of noise letters upon the identification of a target letter in a nonsearch task. Perception \& Psychophysics, 16, 143149.

Felleman, D. J., \& Van Essen, D. C. (1991). Distributed hierarchical processing in the primate cerebral cortex. Cerebral Cortex, 1, 1-47.

Finkelmeyer, A. (2001). Die Rolle der Gravitation beim Aufbau und Abruf räumlichen Wissens. Unpublished Thesis, University of Göttingen.

Fisher, N. I. (1993). Statistical Analysis of Circular Data. Cambridge, MA: Cambridge University Press.

Flanders, M., Helms Tillery, S. I., \& Soechting, J. S. (1992). Early stages in a sensorimotor transformation. Behavioral and Brain Sciences, 15, 309-362. 
Franz, V. H., Gegenfurtner, K. R., Bülthoff, H. H., \& Fahle, M. (2000). Grasping visual illusions: No evidence for a dissociation between perception and action. Psychological Science, 11, 20-25.

Funahashi, S., Bruce, C. J., \& Goldman-Rakic, P. S. (1989). Mnemonic coding of visual space in the monkey's dorsolateral prefrontal cortex. Journal of Neurophysiology, 61, 331-349.

Fuster, J. (1988). The Prefrontal Cortex. $2^{\text {nd }}$ Edition. New York: Raven.

Georgopoulos, A. P., Schwartz, A. B., \& Kettner, R. E. (1986). Neuronal population coding of movement direction. Science, 233, 1416-1419.

Georgopoulos, A. P., Lurito, J. T., Petrides, M., Schwartz, A. B., \& Massey, J. T. (1989). Mental rotation of the neuronal population vector. Science, 243, 234236.

Gescheider, G. A. (1997). Psychophysics: The Fundamentals. $3^{\text {rd }}$ Edition. Mahwah, $\mathrm{NJ}$ : Lawrence Erlbaum Associates.

Gilbert, C. D. (1998). Adult cortical dynamics. Physiological Review, 78, 467-485.

Goldman-Rakic, P. S. (1995). Cellular basis of working memory. Neuron, 14, 477485.

Goldman-Rakic, P. S., Ó Scalaidhe, S. P., \& Chafee, M. V. (2000). Domain specificity in cognitive systems. In M. S. Gazzaniga (Ed.), The New Cognitive Neurosciences (pp. 733-742). Cambridge, MA: MIT Press.

Goodale, M. A., Milner, A. D., Jakobson, L. S., \& Carey, D. P. (1991). A neurological dissociation between perceiving objects and grasping them. Nature, 349, 154156.

Goodale, M. A., \& Milner, A. D. (1992). Separate visual pathways for perception and action. Trends in Neurosciences, 15, 20-25.

Haffenden, A. M., \& Goodale, M. A. (1998). The effect of pictorial illusion on prehension and perception. Journal of Cognitive Neuroscience, 10, 122-136.

Halligan, P. W., \& Marshall, J. C. (1994). Toward a principal explanation of unilateral neglect. Cognitive Neuropsychology, 11, 167-206.

Hershensen, M. (1999). Visual Space Perception: A Primer. Cambridge, MA: MIT Press.

Hollands, J. G., \& Dyre, B. P. (2000). Bias in proportion judgments: The Cyclical Power Model. Psychological Review, 107, 500-524. 
Hubbard, T. L. (1995). Cognitive representation of motion: Evidence for friction and gravity analogues. Journal of Experimental Psychology: Learning, Memory, and Cognition, 21, 241-254.

Huttenlocher, J., Hedges, L. V., \& Duncan, S. (1991). Categories and particulars: Prototype effects in estimating spatial location. Psychological Review, 98, 352-376.

Kohonen, T. (1982). Self-organized formation of topologically correct feature maps. Biological Cybernetics, 43, 59-69.

LaBerge, D. (1995). Attentional Processing: The Brain's Art of Mindfulness. Cambridge, MA: Harvard University Press.

LaBerge, D., \& Brown, V. (1989). Theory of attentional operations in shape identification. Psychological Review, 96, 101-124.

Laeng, B., Peters, M., \& McCabe, B. (1998). Memory for locations within regions: Spatial biases and visual hemifield differences. Memory \& Cognition, 26, 1, 97-107.

Livingstone, M. S., \& Hubel, D. H. (1987). Psychophysical evidence for separate channels for the perception of form, color, movement, and depth. Journal of Neuroscience, 7, 3416-3468.

Livingstone, M. S., \& Hubel, D. H. (1988). Segregation of form, color, movement and depth: anatomy, physiology, and perception. Science, 240, 740-749.

MacMillan, N. A., \& Creelman, C. D. (1991). Detection Theory: A User's Guide. Cambridge: Cambridge University Press.

Marr, D. (1982). Vision. San Francisco: W.H. Freeman.

Marr, D., \& Nishihara, H. K. (1978). Representation and recognition of the spatial organization of three-dimensional shapes. Proceedings of the Royal Society of London, 200, 269-294.

Maxwell, S. E., \& Delaney, H. D. (2000). Designing Experiments and Analyzing Data: A Model Comparison Perspective. Mahwah, NJ: Lawrence Erlbaum Associates.

Merigan, W. H., \& Maunsell, J. H. R. (1993). How separate are the primate visual pathways? Annual Review of Neuroscience, 16, 369-402.

Milner, A. D., Perret, D. I., Johnston, R. S., Benson, P. J., Jordan, T. R., Heeley, D. W., Bettucci, D., Mortara, F., Mutani, R., Terrazzi, E., \& Davidson, D. L. W. (1991). Perception and action in visual form agnosia. Brain, 114, 405-428. 
Milner, A. D., \& Goodale, M. A. (1995). The Visual Brain in Action. Oxford: Oxford University Press.

Moran, J., \& Desimone, R. (1985). Selective attention gates visual processing in extastriate cortex. Science, 229, 782-784.

Müsseler, J., \& Aschersleben, G. (1998). Localizing the first position of a moving stimulus: The Fröhlich effect and an attention-shifting explanation. Perception \& Psychophysics, 60, 683-695.

Nelson, T. O., \& Chaiklin, S. (1980). Immediate memory for spatial location. Journal of Experimental Psychology: Human Learning and Memory, 6, 529-545.

Nijhawan, R. (1994). Motion extrapolation in catching. Nature, 370, 256-257.

Norman, J. F., Todd, J. T., Perotti, V. J., \& Tittle, J. S. (1996). The visual perception of three-dimensional length. Journal of Experimental Psychology: Human Perception and Performance, 22, 173-186.

Palmer, S. E. (1999). Vision Science: Photons to Phenomenology. Cambridge, MA: MIT Press.

Palmer, S. E., \& Bucher, N. M. (1981). Configural effects in perceived pointing of ambiguous triangles. Journal of Experimental Psychology: Human Perception and Performance, 7, 88-114.

Palmer, S. E., \& Bucher, N. M. (1982). Textural effects in perceived pointing of ambiguous triangles. Journal of Experimental Psychology: Human Perception and Performance, 8, 693-708.

Palmer, S. E., \& Rock, I. (1994). Rethinking perceptual organization: The role of uniform connectedness. Psychonomic Bulletin \& Review, 1, 29-55.

Passingham, R. E. (1995). The Frontal Lobes and Voluntary Action. Oxford, UK: Oxford University Press.

Perenin, M. T., \& Vighetto, A. (1988). Optic ataxia: a specific disruption in visuomotor mechanisms. Different aspects of the deficit in reaching for objects. Brain, 111, 643-674.

Phillips, W. A., \& Baddeley, A. D. (1971). Reaction time and short-term visual memory. Psychonomic Science, 22, 73-74.

Psotka, J. (1978). Perceptual processes that may create stick figures and balance. Journal of Experimental Psychology: Human Perception and Performance, 4, 101-111.

Rojas, R. (1993). Theorie der neuronalen Netze. Berlin: Springer. 
Ross, J., Morrone, M. C., \& Burr, D. C. (1997). Compression of visual space before saccades. Nature, 386, 598-601.

Sawaguchi, T., \& Goldman-Rakic, P. S. (1991). D1 dopamine receptors in prefrontal cortex: Involvement in working memory. Science, 251, 947-950.

Schmidt, T., Werner, S., \& Diedrichsen, J. (2001). Spatial distortions induced by multiple visual landmarks: Evidence for a partition principle. Manuscript submitted for publication.

Sekuler, A. B. (1996). Axis of elongation can determine reference frames for object perception. Canadian Journal of Experimental Psychology, 50, 270-278.

Soechting, J. F., \& Flanders, M. (1992). Moving in three-dimensional space: Frames of reference, vectors, and coordinate systems. Annual Review of Neuroscience, 15, 167-191.

Spitzer, H., Desimone, R., \& Moran, J. (1988). Increased attention enhances both behavioral and neural performance. Science, 240, 338-340.

Suzuki, S., \& Cavanagh, P. (1997). Focused attention distorts visual space: An attentional repulsion effect. Journal of Experimental Psychology: Human Perception and Performance, 23, 443-463.

Trommershäuser, J. (2001). Strategieabhängige Verzerrungen im visuell-räumlichen Arbeitsgedächtnis: Ein theoretisch-experimenteller Ansatz. Unpublished Thesis, University of Göttingen.

Trommershäuser, J., \& Schmidt, T. Manuscript in preparation.

Turvey, M. T. (1973). On peripheral and central processes in vision: Inferences from an information-processing analysis of masking with patterned stimuli. Psychological Review, 80, 1-52.

Ungerleider, L. G., \& Mishkin, M. (1982). Two cortical visual systems. In D. J. Ingle, M. A. Goodale, \& R. J. W. Mansfield (Eds.), Analysis of Visual Behavior (pp. 549-586). Cambridge, MA: MIT Press.

van der Heijden, A. H. C., van der Geest, J. N., de Leeuw, F., Krikke, K., \& Müsseler, J. (1999). Sources of position-perception error for small isolated targets. Psychological Research, 62, 20-35.

von der Heydt, R., \& Peterhans, E. (1989). Mechanisms of form perception in monkey visual cortex. I. Lines of pattern discontinuity. Journal of Neuroscience, 9 , 1731-1748. 
von der Malsburg, C. (1973). Self-organisation of orientation-sensitive cells in the striate cortex. Kybernetik, 14, 85.

Wagner, M. (1985). The metrics of visual space. Perception \& Psychophysics, 38, 483-495.

Wandell, B. A. (1995). Foundations of Vision. Sunderland, MA: Sinauer Associates, Inc.

Watson, A. I. (1977). A theory of visual illusions. British Journal of Mathematical and Statistical Psychology, 30, 43-59.

Werner, S. \& Schmidt, T. (2000). Investigating spatial reference systems through distortions in visual memory. In C. Freksa, W. Brauer, C. Habel, \& K.F. Wender (Eds.), Spatial Cognition II: Integrating Abstract Theories, Empirical Studies, Formal Methods, and Practical Applications (pp. 169-183). Berlin: Springer.

Wertheimer, M. (1923). Untersuchungen zur Lehre von der Gestalt. Psychologische Forschung, 4, 301-350.

Zeki, S. (1993). A Vision of the Brain. Oxford, UK: Blackwell. 


\section{Curriculum Vitae}

Name: Thomas Schmidt

Geburtsdatum: $\quad 29$. Februar 1972 in Braunschweig

dienstliche Thomas Schmidt

Anschrift: $\quad$ Georg-August-Universität Göttingen

Georg-Elias-Müller-Institut für Psychologie

Abteilung für Arbeits- und Kognitionspsychologie

Goßlerstr. 14

37073 Göttingen

E-Mail: thomas.schmidt@psych.uni-goettingen.de

Tel.: $\quad 0551 / 39-3612$

Fax: $\quad 0551 / 39-3662$

Homepage: http://kiwi.uni-psych.gwdg.de/home/schmidt/

private Anschrift: Thomas Schmidt

Wiesenstr. 15

37073 Göttingen

Tel. $0551 / 5312617$

Eltern: Hans-Werner Schmidt

Verkaufsberater, in Rente

Ursula Schmidt

Buchhalterin, in Rente

Schulbesuch: $\quad 1978-1982$ Grundschule Comeniusstr., Braunschweig 1982 - 1984 Orientierungsstufe Leonhardstr., Braunschweig 1984 - 1991 Gymnasium Kleine Burg, Braunschweig

Abitur: $\quad$ 04. 06. 1991, Gymnasium Kleine Burg, Braunschweig

Zivildienst: $\quad$ Oktober 1991 - Dezember 1992

Kirchengemeinde St. Johannis, Braunschweig

(ambulante Altenpflege) 
Studium:

Projektarbeit:
SoSe 1993 - SoSe 1997:

April 1993: Immatrikulation an der Technischen Universität CaroloWilhelmina, Braunschweig (Diplomstudiengang Psychologie)

07. 04. 1995: Vordiplom (Note: "Sehr gut")

30. 09. 1997: Diplom (Note: "Sehr gut")

ab SoSe 1995 Tätigkeiten als studentische Hilfskraft in Forschung und Lehrveranstaltungen

Diplomarbeit: "Priming durch metakontrast-maskierte Farbreize:

Wahrnehmung und Reaktionskontrolle ohne Bewußtsein"; betreut von Prof. Dr. Dirk Vorberg und Dr. Klaus Nippert; Note: "Sehr gut"

SoSe 1998 - SoSe 1999:

Ludwig-Maximilians-Universität München

Promotionsstudium der Psychologie

WiSe 1999/2000 - WiSe 2001/2002:

Georg-August-Universität Göttingen

Promotionsstudium der Psychologie

\section{Oktober 1997 - 31. Dezember 1998}

Ludwig-Maximilians-Universität und Max-Planck-Institut für psychologische Forschung (Abteilung "Wahrnehmung und Handlung"), München:

Projektmitarbeiter im DFG-Projekt "Handlungsabhängige Wahrnehmung" bei Prof. Dr. Wolfgang Prinz, Prof. Dr. Josef Zihl und Dr. Harold Bekkering (Ende des Projekts im Oktober 1998).

\section{Januar 1999 - 1. Oktober 2000}

Georg-August-Universität Göttingen, Georg-Elias-Müller-Institut für Psychologie (Abteilung für Kognitions- und Arbeitspsychologie):

wissenschaftlicher Angestellter;

Projektmitarbeiter im DFG-Projekt "Analoge Repräsentation räumlichen Wissens" im DFG-Schwerpunktprogramm "Raumkognition" bei Prof. Dr. Gerd Lüer und Prof. Dr. Steffen Werner.

seit 1. Oktober 2000:

wissenschaftlicher Mitarbeiter 